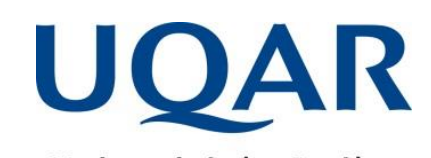

Université du Québec

à Rimouski

\title{
L'ÉTENDUE EFFECTIVE DE LA PRATIQUE DES INFIRMIÈRES DANS LES SERVICES DE PROXIMITÉ EN RÉGION ÉLOIGNÉE
}

\author{
Mémoire présenté \\ dans le cadre du programme de maîtrise en sciences infirmières avec mémoire \\ en vue de l'obtention du grade de Maître ès sciences (M.Sc.)
}

PAR

C MÉLANIE MORIN

Février 2020 

Composition du jury :

Mme Nicole Ouellet Ph.D., présidente du jury, Université du Québec à Rimouski Mme Lily Lessard Ph.D., directrice de recherche, Université du Québec à Rimouski Mme Marie-Ève Poitras Ph.D., examinatrice externe, Université de Sherbrooke 



\title{
UNIVERSITÉ DU QUÉBEC À RIMOUSKI
}

Service de la bibliothèque

\begin{abstract}
Avertissement
La diffusion de ce mémoire ou de cette thèse se fait dans le respect des droits de son auteur, qui a signé le formulaire «Autorisation de reproduire et de diffuser un rapport, un mémoire ou une thèse ». En signant ce formulaire, l'auteur concède à l'Université du Québec à Rimouski une licence non exclusive d'utilisation et de publication de la totalité ou d'une partie importante de son travail de recherche pour des fins pédagogiques et non commerciales. Plus précisément, l'auteur autorise l'Université du Québec à Rimouski à reproduire, diffuser, prêter, distribuer ou vendre des copies de son travail de recherche à des fins non commerciales sur quelque support que ce soit, y compris l'Internet. Cette licence et cette autorisation n'entraînent pas une renonciation de la part de l'auteur à ses droits moraux ni à ses droits de propriété intellectuelle. Sauf entente contraire, l'auteur conserve la liberté de diffuser et de commercialiser ou non ce travail dont il possède un exemplaire.
\end{abstract}



Je dédie ce mémoire de maîtrise à mon conjoint Stéphane ainsi qu'à mes enfants, Tommy et Maïka, à qui j'aimerais transmettre le courage de réaliser leurs rêves... 



\section{REMERCIEMENTS}

D'abord, je tiens à offrir mes remerciements les plus sincères à ma directrice de recherche, Mme Lily Lessard, Ph.D, professeure au département des sciences infirmières de l'Université du Québec à Rimouski (UQAR) (Campus de Lévis) pour son soutien inestimable, sa rigueur scientifique, son professionnalisme, sa présence ainsi que sa patience tout au long de ce parcours. Elle est pour moi un modèle et une mentore qui m'a permis de me développer autant professionnellement que personnellement.

Un merci particulier à Mme Danielle Boucher, Ph.D, professeure et directrice des programmes d'études avancées en sciences infirmières de l'UQAR pour ses nombreux encouragements ainsi que ses judicieux conseils depuis le début de ce projet.

J'offre également mes remerciements à l'ensemble des participants qui ont participé à cette étude. Sans vous, ce projet n'aurait pu être possible.

Merci à tous les gens qui ont travaillé de près ou de loin à la réalisation de ce projet. Merci à mes gestionnaires de m'avoir accordé les congés nécessaires à la poursuite de mes études et de mon projet de recherche. Merci à M. Stéphane Turcotte statisticien au centre de recherche du CISSS Chaudière-Appalaches pour ses précieux conseils en vue de l'analyse des données.

Je veux également remercier le ministère de l'Éducation et de l'Enseignement supérieur (MÉES) ainsi que le Réseau de recherche en interventions en sciences infirmières du Québec (RRISIQ) pour leur soutien financier sous forme de bourses d'études.

Ensuite, je tiens à remercier mon partenaire de vie Stéphane pour son soutien moral, ses encouragements, son écoute, sa compréhension et sa patience à travers tout ce long parcours qu'a demandé ce projet de maîtrise. Merci infiniment mon amour. 
À mes enfants Tommy et Maika de m'avoir permis de consacrer le temps nécessaire à la réalisation de ce projet et de m'avoir soutenue tout au long de ce parcours, merci mes amours.

Merci, merci, merci... 


\section{RÉSUMÉ}

Contexte et objectifs de la recherche. Les infirmières du Québec peuvent contribuer à accroître l'accessibilité aux services de santé et la qualité des soins à condition d'occuper pleinement leur champ de pratique (Mathieu, 2019). Cette étude vise à dresser un portrait de l'étendue de la pratique infirmière dans les services de proximité d'une région du Québec éloignée des grands centres urbains et majoritairement rurale afin d'identifier les stratégies les mieux adaptées pour la favoriser. Les objectifs de recherche visaient plus précisément à : 1) mesurer l'étendue effective de la pratique infirmière (ÉEPI) dans les services de proximité de cette région et 2) déterminer les caractéristiques individuelles, professionnelles, organisationnelles et contextuelles l'influençant. Méthode. Cette étude s'inscrit dans un devis descriptif transversal. 44 infirmières pratiquant dans des services de proximité de la région éloignée à l'étude ont répondu à une adaptation du Questionnaire de l'étendue de la pratique infirmière (D'Amour, Dubois, Déry, Clarke, Tchouaket, Blais \& Rivard, 2012) comprenant 26 questions regroupées en 6 dimensions, soit : 1l'évaluation et la planification des soins; 2- 1'enseignement à la clientèle et aux familles; 3- la communication et la coordination des soins; 4- l'intégration et l'encadrement du personnel; 5- 1'optimisation de la qualité et de la sécurité des soins; et 6- la mise à jour et l'utilisation des connaissances. Résultats et discussion. Le score global de l'étendue effective de la pratique infirmière $(4,15 / 6$; E.T. $=0,85)$ indique une étendue pouvant être améliorée. Les activités associées à la dimension de l'évaluation et la planification des soins $(4,62 / 6$; E.T.= 0,84) obtiennent les scores les plus élevés alors que celles liées à l'optimisation de la qualité et la sécurité des soins $(3,82 / 6$; E.T.=1,14) et l'intégration et l'encadrement du nouveau personnel $(3,4 / 6$; E.T.= 1,30$)$ sont les plus faibles. Certaines caractéristiques telles que le travail à temps plein, le lieu de travail à proximité des grands centres et le fait de détenir le droit de prescrire semblent influencer à la hausse certaines dimensions de l'ÉEPI. Les résultats mettent en lumière des difficultés en lien avec les activités de coordination des soins, la collaboration interprofessionnelle ainsi qu'avec l'expression du leadership clinique.

Mots clés : Étendue de la pratique infirmière; rôle infirmier; services de première ligne; infirmières autorisées; administration des soins infirmiers. 


\begin{abstract}
Context \& research objectives. Quebec nurses can help to increase access to health services and the quality of care provided if they fully occupy their field of practice (Mathieu, 2019). This study aims to draw a portrait of scope of nursing practice in community-based services in a remote region of Quebec, predominantly rural, in order to identify the most appropriate strategies to promote it. More specifically, the research objectives were to 1) measure the actual scope of nursing practice (ASCOP) in community-based services in this region; and 2) determine if individual, professional, organizational and contextual characteristics influence it. Method. This study is based on a descriptive transversal research design. Forty-four (44) nurses practising in communitybased services in the studied remote region responded to an adaptation of the actual scope of nursing practice questionnaire (D'Amour, Dubois, Déry, Clarke, Tchouaket, Blais \& Rivard, 2012) that includes 26 questions grouped into 6 dimensions: 1- assessment and care planning; 2- teaching of patients and families; 3- communication and care coordination; 4- integration and supervision of staff; 5- quality of care and patient safety; and 6- knowledge updating and utilization. Results and discussion. The overall score $(4,15 / 6$, SD $=0,85)$ indicates a suboptimal ASCOP. Activities associated with the assessment and care planning $(4,62 / 6$; E.T. $=0,84)$ score highest while those related to quality of care and patient safety $(3,82 / 6 ;$ E.T.= 1,14$)$ and integration and supervision of staff $(3,4 / 6 ;$ E.T. $=1,30)$ are the lowest. Some characteristics such as working full time, working in areas closer to largest centres and having the right to prescribe seem to have a positive influence on some ASCOP dimensions. The results highlight challenges related to care coordination activities, interprofessional collaboration and the expression of clinical leadership.
\end{abstract}

Keywords: Scope of nursing practice; nursing role; primary health care; registered nurses; nursing administration. 


\section{TABLE DES MATIÈRES}

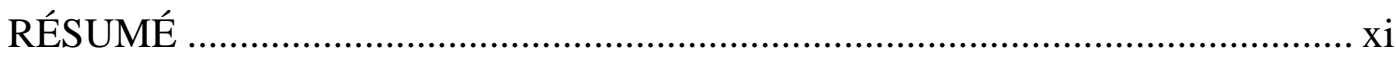

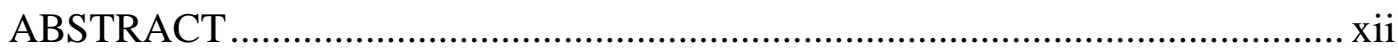

TABLE DES MATIÈRES ……........................................................................ xiii

LISTE DES TABLEAUX ..........................................................................

LISTE DES ABRÉVIATIONS, DES SIGLES ET DES ACRONYMES ............ xix INTRODUCTION GÉNÉRALE _..................................................................... 1

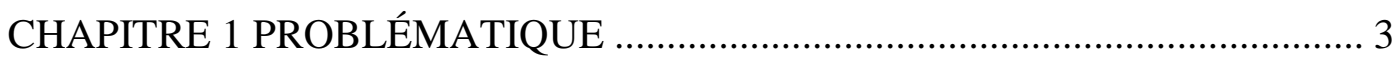

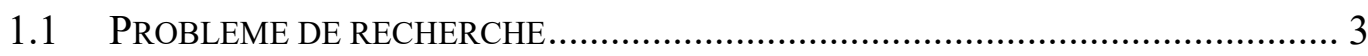

1.1.1 Défi de l'accessibilité aux services de santé en région éloignée au

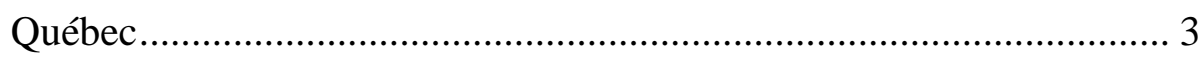

1.1.2 Contribution et étendue de la pratique infirmière ……………………. 7

1.2 PERTINENCE DE LA RECHERCHE POUR LA DISCIPLINE INFIRMIERE .................... 8

CHAPITRE 2 ÉTAT DES CONNAISSANCES SUR L'ÉTENDUE DE LA

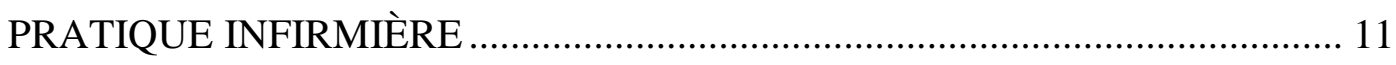

2.1 DÉFINITION DE L'ÉTENDUE DE LA PRATIQUE INFIRMIÈrE.............................. 12

2.2 ÉtUdES AYANT EVALUE L'ETENDUE DE LA PRATIQUE INFIRMIERE ET

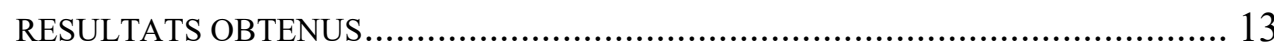

2.3 BÉNÉFICES DE LA PLEINE ÉTENDUE DE LA PRATIQUE DES INFIRMIÈRES EN

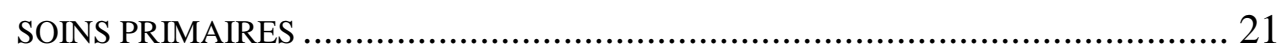

2.3.1 Bénéfices pour les soins de santé, l'organisation et les usagers.......... 21

2.3.2 Bénéfices pour les infirmières.......................................................... 24

2.4 CARACTÉRISTIQUES INFLUENÇANT L'ÉTENDUE DE LA PRATIQUE INFIRMIÈRE ET STRATÉGIES POUR L'ACCROÎTRE …………………………………...... 26

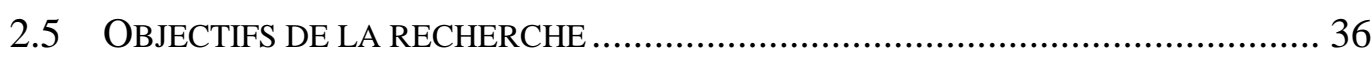

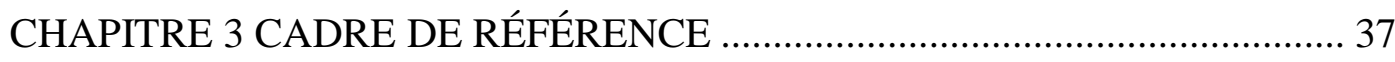




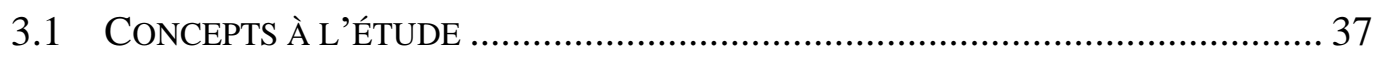

3.1.1 L'étendue de la pratique infirmière ........................................................ 37

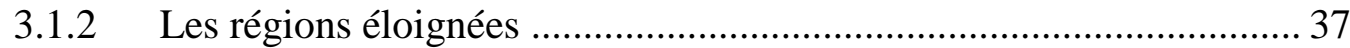

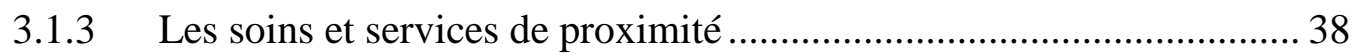

3.1.4 La pratique infirmière dans les services de proximité ……………….... 38

3.1.5 Les caractéristiques influençant l'étendue de la pratique infirmière... 40

3.2 SCHÉMATISATION DES LIENS ENTRE LES CONCEPTS À L’ÉTUDE ...................... 41

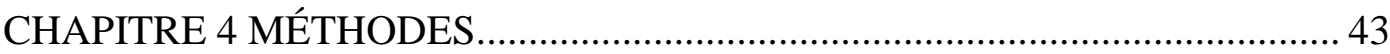

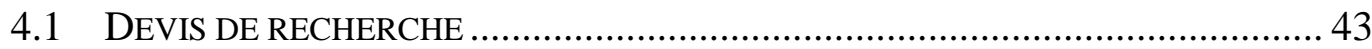

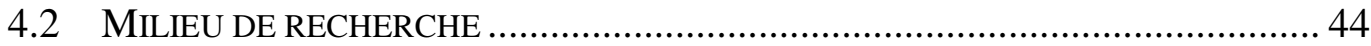

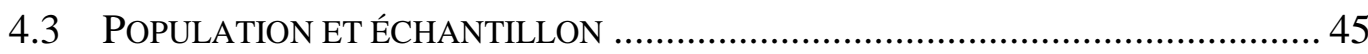

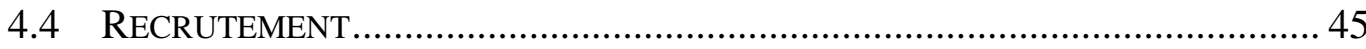

4.5 VARIABLES À L'ÉTUDE ET OUTILS DE COLLECTE DE DONNÉES ....................... 47

4.5.1 Variable dépendante : L'étendue de la pratique des infirmières ......... 47

4.5.2 Variables indépendantes: Caractéristiques individuelles, professionnelles, organisationnelles et contextuelles............................ 49

4.5.3 Biais anticipés et stratégies pour les minimiser.................................. 51

4.6 ANALYSE DES DONNÉES ....................................................................... 51

4.7 CONSIDÉRATIONS ÉTHIQUES ................................................................. 52

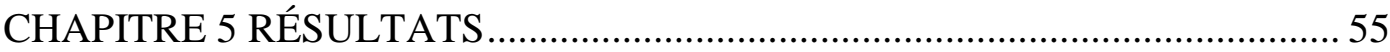

5.1 CARACTÉRISTIQUES DE L'ÉCHANTILLON …………………………............ 55

5.2 EXAMEN DE L'ÉTENDUE EFFECTIVE DE PRATIQUE DES INFIRMIÈRES ..............56

5.3 L'ÉTENDUE EFFECTIVE DE LA PRATIQUE INFIRMIËRES EN FONCTION DES CARACTÉRISTIQUES INDIVIDUELLES, PROFESSIONNELLES, ORGANISATIONNELLES ET CONTEXTUELLES ............................................... 59

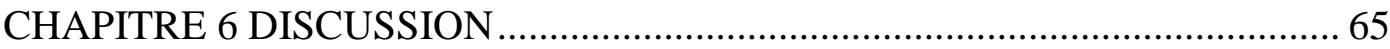


6.1 DISCUSSION DES RÉSULTATS SUR LA MESURE DE L'ÉTENDUE EFFECTIVE DE

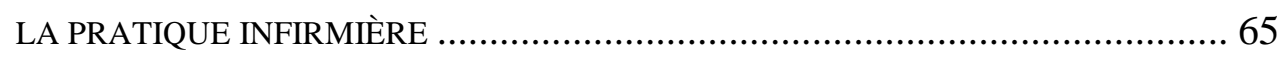

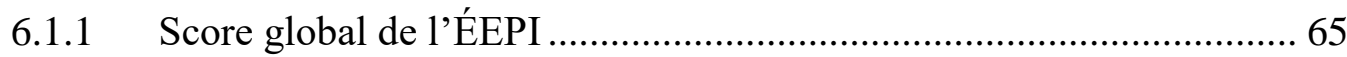

6.1.2 Scores pour chaque dimension de l'étendue de la pratique ................. 66

6.2 DISCUSSION SUR LES EFFETS DES CARACTÉRISTIQUES INDIVIDUELLES, PROFESSIONNELLES, ORGANISATIONNELLES ET CONTEXTUELLES L'ÉTENDUE DE LA PRATIQUE DES INFIRMIÈRES DES SERVICES DE PROXIMITÉ EN RÉGION

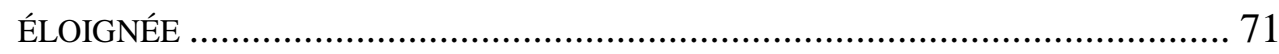

6.2.1 EEPI et droit de prescrire ……………………................................. 71

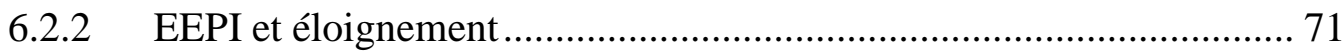

6.2.3 EEPI et formation ........................................................................ 72

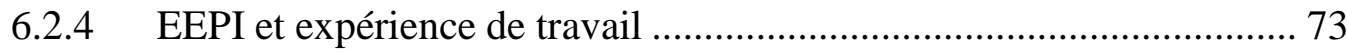

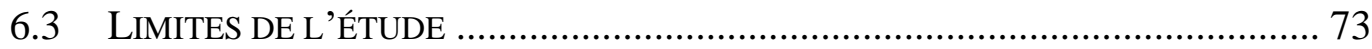

6.4 RECOMMANDATIONS POUR LA FORMATION, LA PRATIQUE, LA GESTION ET LA

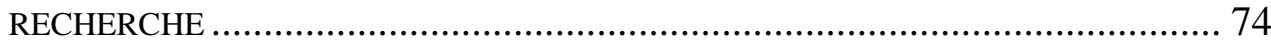

6.4.1 Recommandations pour la formation ............................................... 74

6.4.2 Recommandations pour la pratique clinique …………….................... 75

6.4.3 Recommandations pour la gestion .................................................. 76

6.4.4 Recommandations pour la recherche ................................................. 78

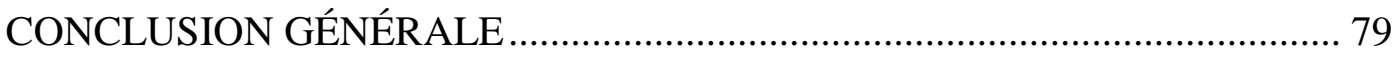

APPENDICE I Soumission de l'article - Revue RSI ........................................... 81

APPENDICE II Activités infirmières réservées des services de proximité (OIIQ,

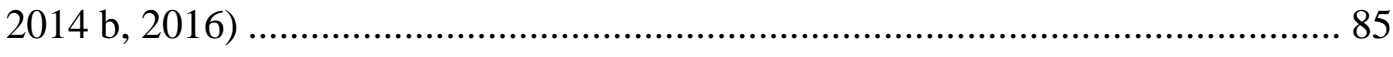

APPENDICE III QÉPI instrument version 2011 …………............................... 91

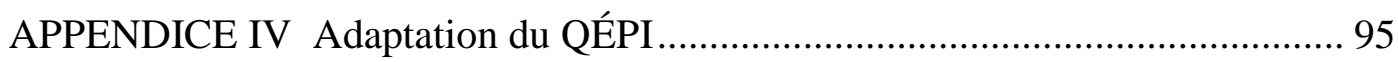

APPENDICE V Certificat éthique ……………………....................................... 101

APPENDICE VI Autorisation du milieu de recherche ....................................... 105 
APPENDICE VII Communication de recrutement.......................................... 109

APPENDICE VIII Formulaire de consentement ............................................ 115

APPENDICE IX Demande de modifications - Certificat éthique...................... 119

APPENDICE X Demande de renouvellement - Certificat éthique ................... 121

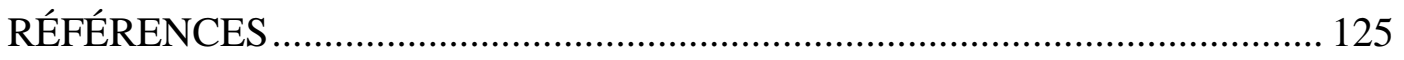




\section{LISTE DES TABLEAUX}

Tableau 1 : Comparaison des scores de l'ÉEPI des études ayant utilisé le QEPI ou ses dérivés ........................................................................................ 18

Tableau 2 : Caractéristiques influençant le déploiement de l'ÉPI selon les études 34

Tableau 3 : Dimensions, items et niveaux de complexité des items du QÉPI ....... 50

Tableau 4 : Résultats de l'ÉEPI globale, de ses dimensions, activités et niveaux de

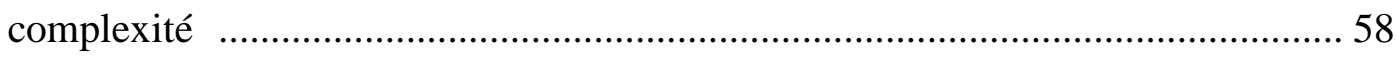

Tableau 5 : Comparaison du score global avec les données sociodémographiques 60

Tableau 6 : Comparaison des différentes dimensions avec les données

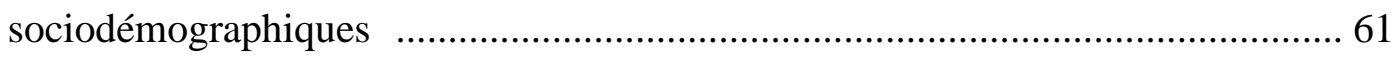

Tableau 7 : Comparaison des niveaux de complexité avec les données

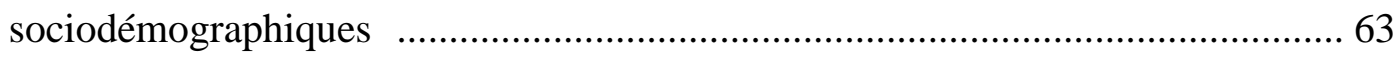




\section{LISTE DES FIGURES}

Figure 1 : Précision des variables indépendantes à l'étude en fonction du cadre de

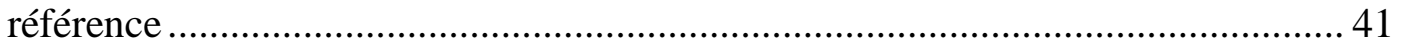




\title{
LISTE DES ABRÉVIATIONS, DES SIGLES ET DES ACRONYMES
}

\author{
ASCOP Actual SCOpe of nursing Practice model. \\ CII Conseil international des infirmières. \\ CISSS Centre intégré de santé et de services sociaux. \\ CIUSSS Centre intégré universitaire de santé et de services sociaux. \\ CLSC Centres locaux de services communautaires. \\ CSBE Commissaire à la santé et au bien-être. \\ ÉEPI Étendue effective de la pratique infirmière. \\ ÉPI Étendue de la pratique infirmière. \\ GMF Groupe de médecine familiale. \\ GMF-U Groupe de médecine familiale universitaire. \\ IPS-PL Infirmière praticienne spécialisée en première ligne. \\ INSPQ Institut national de santé publique du Québec. \\ IOM Institute of medecine. \\ MRC Municipalité régionale de comté. \\ MSSS Ministère de la Santé et des Services sociaux. \\ OIIQ Ordre des infirmières et infirmiers du Québec. \\ OMS Organisation mondiale de la santé. \\ QÉPI Questionnaire de l'étendue de la pratique infirmière
}





\section{INTRODUCTION GÉNÉRALE}

L'accessibilité aux soins de santé présente un défi constant pour les systèmes de soins de toutes les régions du Québec et requiert des solutions efficaces, pragmatiques et adaptées à chaque contexte.

Les infirmières seraient en mesure d'apporter une contribution importante afin d'accroître cette accessibilité à condition toutefois d'être en mesure de déployer l'ensemble de leurs compétences par l'utilisation pleine et entière de leur champ de pratique. Des préoccupations sont toutefois présentes quant au sous-déploiement actuel de la pratique des infirmières (Tremblay, 2018 ; Mathieu, 2019). L'étude présentée dans ce mémoire s'intéresse donc à l'étendue effective de la pratique infirmière (ÉEPI), qui fait référence à l'éventail des fonctions et responsabilités déployées par l'infirmière en lien avec les compétences développées à travers sa formation et son expérience (D’Amour et al., 2012 ; Déry, 2013) dans les services de proximité d'une région majoritairement rurale du Québec et éloignée des grands centres urbains.

En premier lieu, le chapitre 1 présentera la problématique qui aborde le problème de recherche soit le défi de l'accessibilité aux services de santé en région éloignée au Québec, la contribution et l'étendue de la pratique infirmière (ÉPI) et la pertinence de cette recherche pour la discipline infirmière. Le chapitre 2 sera, pour sa part, consacré à l'état des connaissances. À l'intérieur de celui-ci sera présentée la définition de l'étendue de la pratique infirmière, les bénéfices d'une pratique infirmière « étendue » ainsi que les facteurs influençant l'ÉPI et les stratégies pour l'accroître. Ce chapitre se terminera avec la présentation des objectifs de l'étude. Le chapitre 3 est consacré au cadre de référence où les principaux concepts à l'étude sont définis et mis en relation dans une schématisation. Le chapitre 4 porte sur la méthode, soit le devis utilisé, le milieu sélectionné, la population et l'échantillon, le recrutement, la définition des variables à l'étude, la présentation de l'outil de collecte de données, les biais anticipés et les stratégies 
pour les minimiser, l'analyse des données et les considérations éthiques. Bien que les résultats de cette étude aient fait l'objet d'un article paru dans le numéro 138 de septembre 2019 de la revue Recherche en soins infirmiers (Morin et Lessard, 2019), compte tenu des modifications demandées par le comité d'évaluation du présent mémoire, il a été choisi de présenter les résultats et la discussion dans le cadre de deux chapitres classiques. Ainsi, le chapitre 5 présentera les résultats, soit les caractéristiques des répondants, le score global de l'ÉEPI ainsi que les scores de chacune des dimensions et niveaux de complexité. Celui-ci se terminera par la présentation des scores obtenus pour les caractéristiques individuelles, professionnelles, organisationnelles et contextuelles. La discussion des résultats en regard des études sur le sujet, les recommandations pour la recherche, la formation, la pratique et la gestion et pour terminer, les limites de l'étude seront exposées à l'intérieur du sixième et dernier chapitre. La conclusion terminera ce mémoire. 


\section{CHAPITRE 1 \\ PROBLÉMATIQUE}

Ce chapitre présente le problème de recherche en précisant les problématiques d'accès aux services de santé dans les régions éloignées et les moyens pouvant être déployés par les infirmières pour y répondre en abordant plus particulièrement le sujet de l'étendue de leur pratique. Il se conclut avec la pertinence de la recherche pour la discipline infirmière.

\subsection{Probleme de RECHERCHE}

Cette section présente le problème de recherche, soit le défi de l'accessibilité aux services de santé en région éloignée au Québec ainsi que la contribution et étendue de la pratique infirmière.

\subsubsection{Défi de l'accessibilité aux services de santé en région éloignée au Québec}

Au Québec, le vieillissement de la population jumelé à la prévalence croissante de certaines conditions de santé, tels les maladies chroniques et les problèmes de santé mentale, contribue à l'accroissement des besoins de santé de la population (ministère de la Santé et des Services sociaux [MSSS], 2019). Les défis sont donc importants pour assurer l'accessibilité aux soins de santé à toute la population et pour dispenser des services de santé de qualité et efficients dans les différentes régions du Québec. Ces enjeux se reflètent d'ailleurs dans l'une des cibles du MSSS (Gouvernement du Québec, 2017)

qui est d'augmenter l'accessibilité aux soins de proximité. Ces soins sont définis comme le premier point de contact avec le système de santé et réfèrent à deux fonctions, soit de : 
«1- permettre de fournir directement les services de santé de première ligne et 2-permettre de coordonner les services de santé fournis aux patients pour assurer la continuité des soins et faciliter le cheminement au sein du système de soins de santé lorsque des soins plus spécialisés sont nécessaires » (Gouvernement du Canada, 2018).

Afin de répondre à ces défis sociétaux et accroître l'accessibilité aux services de santé de proximité, le gouvernement du Québec a beaucoup misé, ces dernières années, sur le développement de la première ligne médicale. Il a ainsi adopté, en 2015, le projet de loi 20, visant à accroître l'accès aux services de médecine familiale au Québec. Des mesures, comme la création de guichets d'accès et des supercliniques, l'augmentation des inscriptions auprès de médecins de famille ainsi que des incitatifs pour accroître la disponibilité des heures de rendez-vous médicaux, ont été tentées, mais n'ont pas nécessairement atteint les résultats escomptés. Ainsi, la cible provinciale d'inscription auprès d'un médecin de famille qui était de $85 \%$ n'était toujours pas atteinte en 2017 selon le Commissaire à la santé et au bien-être (CSBE) (2017a). De plus, cette inscription ne serait pas garante d'une rapidité d'accès puisque $88 \%$ des médecins québécois n'ont pas pu offrir un rendez-vous à leur clientèle le jour même ou le jour suivant (CSBE, 2017a). Des efforts ont également été déployés afin d'accroître la contribution d'autres professionnels dans les groupes de médecine familiale (GMF) tels que les travailleurs sociaux, les pharmaciens et les nutritionnistes, et ce, afin d'augmenter l'intégration des soins et services de santé physique et sociale (MSSS, 2018). L'institut national d'excellence en santé et en services sociaux (INESSS) a, quant à lui, procédé à la création d'ordonnances collectives nationales permettant une meilleure prise en charge des patients atteints de maladies chroniques par les infirmières en GMF ou dans d'autres contextes des soins primaires, ce qui favorise une meilleure accessibilité aux soins et services de santé et permettrait, selon l'OIIQ, que les infirmières occupent mieux leur champ de pratique (Ordre des infirmières et infirmiers du Québec [OIIQ], 2019a). Finalement, le MSSS vise à rehausser le nombre d'infirmières praticiennes spécialisées (IPS) au Québec et de nouveaux privilèges ont été accordés aux pharmaciens afin d'élargir leurs activités. 
Il est normalement attendu que «les modèles organisationnels conçus pour l'ensemble du Québec doivent invariablement être adaptés aux particularités des contextes ruraux et éloignés » (Gauthier et al., 2009, p.I). Or, les mesures adoptées depuis 2015 afin d'accroître l'accessibilité ne tiennent pas toujours compte des réalités de certaines régions éloignées du Québec où l'immensité de certains territoires ainsi que la faible densité de population présentent des défis constants dans la dispensation des services de santé et des services sociaux, notamment sur le plan des ressources, tant humaines que financières. Les CISSS et CIUSSS du Québec, créés en 2015 suite à la fusion des agences de santé et de services sociaux et des établissements de santé publics de chacune des régions, sont responsables d'offrir les services de santé et services sociaux sur de vastes territoires. Le CISSS de la Côte-Nord remporte la palme du plus grand territoire, suivi par le CIUSSS $\mathrm{du}$ Saguenay-Lac-Saint-Jean et le CISSS de l'Abitibi-Témiscamingue avec respectivement $236665 \mathrm{~km}^{2}$ équivalents à la taille du Royaume-Uni avec une population totale de 91213 habitants (densité de population de 0,4 hab./ $\mathrm{km}^{2}$ ) pour la Côte-Nord, $95760 \mathrm{~km}^{2}$ avec une population de 277406 habitants (densité de population $2.9 \mathrm{hab} . / \mathrm{km}^{2}$ ) pour le Saguenay-Lac-Saint-Jean et $57349 \mathrm{~km}^{2}$ avec une population de 147508 habitants (densité de population de 2,6 hab./ $\mathrm{km}^{2}$ ) pour 1'Abitibi-Témiscamingue (Institut de la statistique du Québec [ISQ], 2019).

Ces régions éloignées vivent souvent des problèmes d'attraction et de rétention des professionnels de la santé, plusieurs citoyens doivent consulter des médecins itinérants auprès de qui ils ne peuvent pas s'inscrire. D'autres compteront sur des dispensaires infirmiers comme premier niveau de soins. De plus, dans ces régions, les médecins généralistes sont amenés à diviser leurs temps de pratique entre leur cabinet et les autres structures du système de soins (par ex. : hospitalisation, gardes hospitalières, urgence, oncologie, soutien à domicile, centres d'hébergement et de soins de longue durée, etc. (Savard et Rodrigue, 2001). La présence médicale en première ligne est donc contrainte par la nécessité de satisfaire tous ces besoins institutionnels à même les effectifs disponibles. Ces enjeux d'accessibilité occasionnent un achalandage plus grand dans les services d'urgence pour des conditions ne nécessitant pas ce niveau de soins (Gauthier et 
al., 2009; CSBE, 2017b). Ils peuvent aussi entraîner des besoins non comblés de la population à l'égard du suivi des patients souffrant de maladies chroniques (CSBE, 2014, 2017b). Ainsi, la proportion des visites avec un niveau de priorité 4 (moins urgent) ou 5 (pas urgent) et la proportion de celles sans prise en charge médicale qui est de $59 \%$ des visites aux services d'urgence pour l'ensemble du Québec, se situent plutôt à respectivement $79 \%, 82 \%$ et $84 \%$ pour l'Abitibi-Témiscamingue, la Côte-Nord et la Gaspésie (CSBE, 2017b). Dans ces régions éloignées, la proportion des visites à l'urgence avec un niveau de priorité 4 ou 5 au Québec est demeurée assez stable depuis 2014 avec une variation de $-0,2 \%$ pour la Côte-Nord et $-0,4 \%$ pour l'Abitibi-Témiscamingue alors que pour l'ensemble du Québec elles ont diminué de plus de $2 \%$. (CSBE, 2017 b). En ce qui concerne la proportion des visites à l'urgence sans prise en charge médicale, elle est même en augmentation dans certaines régions éloignées avec 6,9\% pour l'AbitibiTémiscamingue, 1,5 \% pour la Côte-Nord et 2,2 \% pour le Bas-Saint-Laurent alors que l'ensemble du Québec présente une diminution de 5,2\% en comparaison avec 2014 (CSBE, 2017b).

En région éloignée, le constat des effets limités des mesures touchant uniquement les médecins, comme celles proposées dans le projet de loi 20, indique que la recherche de solutions doit se poursuivre pour y améliorer l'accès aux soins de proximité. Bien que l'intégration des infirmières praticiennes spécialisées de première ligne (IPS-PL) au Québec est présentée comme une mesure permettant de renforcer la première ligne et d'accroître l'accessibilité aux soins et services de santé, l'absence de programme universitaire de deuxième cycle d'IPS-PL dans certaines de ces régions éloignées, comme la Côte-Nord et la Gaspésie constituent des freins non négligeables à l'engagement des infirmières de ces régions dans ces cheminements professionnels, car elles doivent s'expatrier pour étudier sans forcément garantir un retour en région une fois la scolarité terminée. Le recrutement d'IPS-PL diplômée dans d'autres régions est également un défi pour les régions éloignées. Ainsi, bien qu'il soit d'une grande importance de poursuivre les travaux visant la bonification de l'offre de services universitaires et le recrutement des IPS en région, d'autres solutions doivent être envisagées afin de répondre aux besoins de 
santé de la population. Les infirmières et les infirmières cliniciennes sont une ressource disponible dans les régions éloignées, elles peuvent également contribuer à améliorer l'accès aux soins et services à condition d'occuper pleinement leur champ de pratique.

\subsubsection{Contribution et étendue de la pratique infirmière}

Selon 1'Institute of medicine (IOM), les infirmières développent des compétences permettant de contribuer à l'accessibilité aux soins, de coordonner des soins de plus en plus complexes et d'optimiser leur pratique en soins primaire en respect de leur formation (Institute of Medicine (IOM), 2010). Il a d'ailleurs été démontré que ces dernières amélioraient les soins fournis aux patients souffrant de maladies chroniques (Bauer et Bodenheimer, 2017). L'ajout d'infirmières exerçant pleinement leur rôle contribuerait, de plus, à une économie de coûts et à une meilleure qualité et sécurité des soins (Smolowitz, Speakman, Wojnar, Whelan, Ulrich, Hayes et Wood, 2015; OIIQ, 2014a; Comité de la formation des infirmières, 2014, 2016).

Les infirmières pourraient donc représenter une partie de la solution aux problèmes d'accessibilité et de continuité des soins et services de santé, à condition de pouvoir occuper pleinement leur champ de pratique (Normandin, 2016; OIIQ, 2014a). Certains auteurs ont également mis en lumière l'importance du plein rôle infirmier dans les soins de santé primaires, notamment en région (Parkinson et Parker, 2013). Ceux-ci écrivent à ce sujet que : «d'autres professionnels de la santé (que les omnipraticiens) sont tenus de répondre à la demande croissante de services et de faire face aux pénuries de personnels de santé, en particulier dans les zones rurales, isolées et régionales »[traduction libre] (Parkinson et Parker, 2013, p.591). Une utilisation judicieuse des ressources infirmières contribuerait également à diminuer les coûts de fonctionnement ainsi que les problèmes de pénurie de main-d'œuvre au sein du réseau de la santé du Québec (Besner, Doran, McGillis Hall, Giovannetti, Girard, Hill et Morrison, 2005). Également, cette pleine 
étendue de la pratique infirmière serait associée à une plus grande satisfaction professionnelle (Déry, 2013). Ce dernier aspect est non-négligeable dans des régions éloignées qui éprouvent d'importants problèmes d'attraction et de rétention de la maind'œuvre infirmière. Par exemple, dans la région de la Côte-Nord, de 2009 à 2014, le nombre total d'infirmières a chuté de $17 \%$ (ISQ, 2015).

Malgré tous ces avantages, des études québécoises, canadiennes ou d'ailleurs dans le monde mettent en lumière une sous-utilisation de la main-d'œuvre infirmière et un déploiement de pratique sous-optimal en milieux hospitaliers (Besner et al., 2005; White, Oelke, Besner, Doran, Hall et Giovannetti, 2008; Oelke, White, Besner, Doran, Hall et Giovannetti, 2008; D’Amour et al., 2012; Déry, 2013; Lafleur, 2016) ainsi que dans les

milieux de première ligne (Braithwaite, 2016). L'Organisation mondiale de la santé (OMS) a appelé à la levée des obstacles empêchant les infirmières de pratiquer à leur plein potentiel (Büscher, Sivertsen et White, 2009). Il apparaît donc urgent de remédier aux problèmes à l'origine de cette situation, notamment dans les services de proximité des régions éloignées.

Cette problématique mène à l'interrogation suivante : La pratique des infirmières travaillant dans les services de proximité en région est-elle optimale pour leur permettre de bien contribuer à améliorer l'accessibilité et la continuité des soins et services en région? Une recherche sur le sujet permettra de mieux comprendre ce phénomène afin de suggérer des modifications adaptées visant à accroître l'étendue de leur pratique notamment en région éloignée.

\subsection{PERTINENCE DE LA RECHERCHE POUR LA DISCIPLINE INFIRMIERE}

L'intérêt pour l'étendue de la pratique des infirmières ne date pas d'hier. La pratique infirmière s'est grandement élargie au cours des 15 dernières années grâce à différents leviers légaux. En effet, deux projets de loi sont venus modifier le code des professions 
soit les projets de loi 90 et 21 adoptés respectivement à la fin 2002 et en 2009. Ces modifications ont permis un nouveau partage des champs d'exercices des professionnels dans le domaine de la santé et de spécifier les activités réservées aux infirmières. Également, un règlement adopté par le Gouvernement du Québec (2016) en collaboration avec le Collège des médecins du Québec en vertu du Règlement sur certaines activités professionnelles qui peuvent être exercées par une infirmière et un infirmier permet, depuis le 11 janvier 2016, à l'ensemble des infirmières titulaire d'un baccalauréat de prescrire des traitements et des examens de laboratoire dans certains domaines tels la santé publique, les problèmes de santé courants ainsi que les soins de plaies. Une clause transitoire permettait, jusqu'au 30 juin 2017, à l'infirmière titulaire d'un diplôme d'études collégiales en soins infirmiers qui exerçait, selon une ordonnance collective, des activités visées en soins des plaies et en santé publique de poursuivre celles-ci. Ces nouvelles perspectives ouvrent la voie à des pratiques infirmières plus autonomes. Ces changements requièrent toutefois des connaissances actuelles issues de la recherche afin d'évaluer le déploiement de la pratique ainsi que ses possibles obstacles dans le but d'apporter des solutions adaptées.

Le sujet de l'étendue de la pratique demeure très actuel. Il est d'ailleurs à l'origine des préoccupations formulées par l'OIIQ dont le mandat est de protéger le public et amène l’organisation à en faire une priorité.

Votre ordre professionnel poursuivra son plan d'action amorcé en 2018 visant ainsi une occupation pleine et entière du champ d'exercices infirmier. Dans ce contexte, il est important de faire rayonner notre profession et de démontrer que les compétences infirmières sont la clef de voûte pour un meilleur accès aux soins de santé. (Mathieu, 2019, paragr. 1 et 2).

Le contexte de la pratique infirmière en région éloignée a toutefois été peu étudié au Québec à ce jour.

Il importe d'aborder ce sujet sous l'angle de la recherche puisque cette dernière est cruciale dans l'émergence et le développement d'une discipline. En effet, la recherche en sciences infirmières permet d'élaborer des savoirs spécifiques et distincts à la discipline 
infirmière (Pepin, Larue, Allard \& Ha, 2015). « Sans recherche, le nursing ne peut pas être une discipline »(Lanara, 1996, p.91 dans Pepin et al., 2015). La production de connaissance en référence aux contextes éloignés permettra de lever le voile sur les particularités de la pratique infirmière dans ces milieux et contribuera à illustrer la variété des contextes de pratique des infirmières. Mieux connue et reconnue, la pratique en milieu éloignée sera davantage considérée dans les prises de décision visant à améliorer l'accès, la qualité et la sécurité des soins pour l'ensemble des Québécois.

Les résultats permettront notamment aux infirmières en situation de gestion de mieux connaître les pratiques des infirmières ainsi que les facteurs les influençant dans le but d'en permettre un meilleur déploiement. Cette recherche participera également à répondre à certaines questions au sujet de la pratique infirmière ainsi qu'à la transformation des systèmes de santé locaux, en interdépendance avec les systèmes internationaux (Pepin et al., 2015, p.40) à l'intérieur des recommandations qui seront émises au milieu par la publication et la diffusion de ce mémoire. Le désir de rayonnement international explique le choix d'avoir publié les résultats de cette recherche dans un article scientifique (Morin et Lessard, 2019) ce qui pourra contribuer à l'avancement de la discipline infirmière au Québec et ailleurs. 


\section{CHAPITRE 2 \\ ÉTAT DES CONNAISSANCES SUR L'ÉTENDUE DE LA PRATIQUE INFIRMIÈRE}

Ce chapitre présente l'état des connaissances sur l'étendue de la pratique infirmière. Il débute par la définition conceptuelle de l'étendue de la pratique infirmière, suivie de la présentation des études ayant évalué l'ÉPI ainsi que les bénéfices d'une pratique infirmière « étendue » notamment en soins primaires, pour la qualité des soins et la satisfaction professionnelle. Ce chapitre se poursuit en exposant les caractéristiques influençant l'ÉEPI et les stratégies pour l'accroître. Il se termine avec la présentation des objectifs et le cadre de référence de cette étude.

Une revue narrative de la littérature a été réalisée à partir de plusieurs bases de données: CINAHL, MEDLINE, EM Premium, ScienceDirect, ProQuest, Papyrus, Corpus, Érudit et Google Scholar en combinant les mots clés « scope of nursing practice » ET « registered nurse ». Ce dernier terme a été retenu, car une première exploration de la littérature avait permis de constater que de nombreuses recherches sur l'optimisation de la pratique des infirmières portaient surtout sur les infirmières praticiennes spécialisées (Kunic et Jackson, 2013; Poghosyan, Boyd, \& Clarke, 2016; Villegas et Allen, 2012) alors que la présente étude s'intéresse aux infirmières et infirmières cliniciennes. Les mots clés faisant référence aux soins de proximité tels que «primary care » et «community care » n'ont pas été retenus puisque trop peu d'études sur l'étendue de la pratique infirmières concernaient ces types de soins et services. Les articles retenus répondaient donc aux critères d'inclusion étaient donc les suivants : 1) articles de 2005 à 2019,2) de langue française ou anglaise et 3) devait concerner les infirmières. Une recherche manuelle à partir des listes de références des articles retenus a été réalisée par la suite. Un premier exercice de repérage des écrits a été réalisé en 2015 puis il a été actualisé chaque année par la suite. Au total, 35 articles ont été retenus. 


\subsection{DEFINITION DE L'ETENDUE DE LA PRATIQUE INFIRMIERE}

Au début des années 2000, Baranek (2005) et Besner et al. (2005) ont indiqué que les définitions de l'ÉPI variaient beaucoup. Selon le Conseil international des infirmières (CII) l'étendue de pratique réfère au cadre législatif qui définit le rôle, les compétences (connaissances, habilités et attitudes) et la responsabilité professionnelle de l'infirmière (CII, 2013). L'American Nurses Association (2017) la décrit plutôt sous l'angle de l'éventail des services qu'un professionnel de la santé peut dispenser détenant la formation, la compétence ainsi que l'autorisation requise conformément aux conditions de sa licence professionnelle, pour les effectuer. Au Canada, plusieurs ordres et associations professionnelles semblent s'entendre pour définir l'étendue de pratique des infirmières comme le déploiement du rôle, de fonctions et de responsabilités de l'infirmière en lien avec les compétences développées à travers l'expérience et la formation (College of Registered Nurses of British Columbia, 2014; College of Registered Nurses of Manitoba, 2010; College of Registered Nurses of Nova Scotia, 2009). Au Québec, l'Ordre des infirmières et infirmiers du Québec (OIIQ) réfère à la définition de D’Amour et al. (2012) et Déry (2013) présentée dans le paragraphe suivant.

Des auteurs apportent des nuances à ces définitions en différenciant l'ÉPI optimale de l'ÉPI effective (ÉEPI). L'ÉPI optimale représente la portée totale de la pratique des infirmières et comprend l'ensemble des activités pour lesquelles elles ont été formées et sont autorisées à exercer (Baranek, 2005) alors que l'ÉEPI est définie comme l'éventail des fonctions et responsabilités déployées par l'infirmière en lien avec les compétences développées à travers la formation et l'expérience (D’Amour et al., 2012 ; Déry, 2013). Une étude du concept de plein potentiel infirmier ( «full nursing potential ») réalisé par Aroke (2014) a mené au développement d'un modèle présentant les facteurs influençant l'ÉPI, les stratégies pour l'accroître ainsi qu'une définition opérationnelle du plein potentiel infirmier qu'il définit comme : 
Un état dynamique dans lequel les règlements, les politiques institutionnelles, la formation, l'expérience, les facteurs environnementaux et les traits personnels contribuent au plein potentiel infirmier, permettant aux infirmières de dispenser des soins de qualité, centrés sur les patients et de promouvoir des systèmes sociaux équitables (Aroke, 2014, page 278).

Le concept de plein potentiel infirmier réfère au déploiement possible de la pratique alors que l'ÉEPI réfère au déploiement réel mesuré. Cette différence entre l'ÉPI

optimale et l'ÉEPI a aussi été mise en évidence dans les travaux d'autres auteurs tels que Besner et al. (2005) dans leur étude sur la perception des infirmières par rapport à l'étendue de leur pratique et aux facteurs l'influençant ainsi que par White et al. (2008) dans leur étude sur la signification qu'accordent les infirmières à l'expression : « travailler à la pleine étendue ».

\section{2 ÉTUDES AYANT EVALUE L'ETENDUE DE LA PRATIQUE INFIRMIERE ET RESULTATS OBTENUS}

Dans cette section, les différentes études d'ici et d'ailleurs portant sur l'étendue de la pratique infirmière ou l'ayant évalué seront présentées. Un intérêt sera porté aux résultats des études et aux différentes dimensions évaluées. Dans un premier temps, les études qualitatives seront présentées suivies par celles fondées sur des méthodes quantitatives.

Une étude menée par Besner et al. (2005) visait à documenter la perception des infirmières par rapport à l'étendue de leur pratique et à identifier les obstacles et les facilitateurs perçus à l'optimisation de leurs rôles. En employant un devis qualitatif descriptif, comparatif exploratoire, 167 infirmières de 14 unités de soins de courte durée (contexte hospitalier) de trois villes, soit Calgary, Edmonton et Saskatoon ont participé à une entrevue individuelle semi-structurée. Plus précisément, il s'agissait d'infirmières autorisées $(n=85)$, d'infirmières auxiliaires $(n=31)$, d'infirmières psychiatriques $(n=11)$, des gestionnaires cliniques $(n=19)$ et d'infirmières dans des rôles spécialisés $(n=21)$. 
L'analyse des entrevues a mis en lumière une difficulté des participants à décrire l'étendue de la pratique infirmière, une utilisation sous-optimale de celle-ci ainsi que certains facteurs l'influençant. En 2008, White et al. (2008) ont réalisé une analyse secondaire des entrevues menées précédemment dans l'étude de Besner et al. (2005) afin de comprendre la signification qu'accordent les infirmières à l'expression: «travailler à la pleine étendue ». Les résultats démontrent que « l'étendue de pratique » est perçue comme les activités réellement réalisées dans le travail quotidien et que la formation, l'expérience et les compétences sont reconnues comme ayant une influence sur son déploiement. D'importantes difficultés ont été rencontrées toutefois par les répondants pour différencier les concepts de «pleine étendue de pratique » et celui «d'étendue réelle de la pratique ». Les résultats ont également révélé l'incapacité d'énoncer clairement des différences entre les rôles des infirmières autorisées, des infirmières autorisées en psychiatrie et des infirmières auxiliaires, et ce malgré d'importantes différences dans la formation, les connaissances et les compétences de ces trois groupes. Les résultats ont donc mis en lumière cette confusion des rôles ainsi que le chevauchement de ceux-ci avec ceux d'autres professionnels comme étant un obstacle important au déploiement de la pratique infirmière. Puisque cette étude portait uniquement sur la pratique des infirmières en soins de courte durée, les auteurs recommandent davantage de recherche afin de déterminer si les problèmes identifiés dans cette étude entravent également l'étendue de la pratique infirmière dans d'autres secteurs de soins tels que le secteur des soins communautaires ou celui de longue durée.

Lampron a conduit une étude qualitative de type descriptive et interprétative visant à décrire l'étendue de la pratique des infirmières et des infirmières cliniciennes dans une unité de médecine et de chirurgie et d'identifier les facteurs organisationnels influençant l'étendue de la pratique. Des entrevues individuelles semi-structurées ont été réalisées auprès de 8 informateurs clés soit trois infirmières et trois infirmières cliniciennes d'une unité de médecine et de chirurgie et de deux gestionnaires. Les résultats démontrent que le déploiement des différentes composantes de l'étendue de la pratique est similaire chez les infirmières détenant ou non un baccalauréat. Toutefois, certaines différences ont été 
recensées quant aux dimensions du leadership et du transfert des connaissances, semblant davantage mobilisées chez les infirmières cliniciennes. L'étude a également permis d'identifier certains facteurs organisationnels qui semblent influencer l'étendue de la pratique telle que la charge de travail, l'environnement de travail et la composition et les caractéristiques des équipes de soins (Lampron, 2010).

Plusieurs études quantitatives ont aussi été repérées. Ainsi, une étude menée par White, Jackson, Besner \& Norris (2015) et fondée sur l'observation du temps passé par les infirmières spécialisées dans la responsabilisation des rôles cliniques clés et d'autres activités professionnelles. Des observateurs qualifiés ont observé un échantillon de convenance d'infirmières autorisées dans deux unités médicales (unités A et B) d'un grand hôpital tertiaire de Calgary, en Alberta. Les aides-soignants ont également été inclus dans la base de sondage, car la composition du personnel infirmier des unités comprenait des infirmières autorisées et des aides-soignants. Les observateurs ont enregistré les activités de 35 infirmières autorisées et de 17 aides-soignants (39 individus uniques) durant leurs quarts de travail seconde par seconde sur une période de 5 jours. Les deux unités ont eu recours à un modèle de soins infirmiers primaires modifié consistant à attribuer un nombre précis de patients à un fournisseur de soins infirmiers réglementé, qui était responsable de tous les soins fournis aux patients qui leur avaient été attribués. Les données indiquaient que, dans les unités $\mathrm{A}$ et $\mathrm{B}$, environ $21 \%$ du temps de l'infirmière autorisée était consacré à l'examen de la documentation et de l'information. Les infirmières ont consacré environ $13 \%$ du temps aux interventions infirmières individuelles telles que les activités de la vie quotidienne / les soins personnels, $12 \%$ pour les médicaments et les traitements. Le temps consacré à l'évaluation / surveillance biomédicale $(8,0$ et 7,1\%) était plus long que celui consacré à l'évaluation / à la surveillance psychosociale culturelle spirituelle du patient et de sa famille. Le fait de combiner évaluation / surveillance psychosociale culturelle spirituelle avec soutien psychosocial culturel spirituel et enseignement aux patients a néanmoins révélé que le temps consacré à la responsabilisation de ce rôle $(4,6$ et 5,0\%) était limité. Comme les soins infirmiers sont un processus collaboratif, on s'attendait à ce qu'une grande partie du temps consacré aux soins infirmiers soit consacrée à la 
coordination des activités de soins (le temps réellement consacré était de 12,6\% et 18,7\%). Les données ont également révélé qu'une quantité substantielle de temps des infirmières autorisées était consacrée à des activités telles que les déplacements (12,8 et 12,6\%). Les infirmières autorisées ont consacré beaucoup de temps à l'évaluation et à la surveillance biomédicales, relativement peu à l'évaluation / à la surveillance psychosociale culturelle spirituelle du patient et de sa famille, ainsi qu'à leur soutien (White et al., 2015).

Plusieurs travaux repérés utilisent un outil appelé le questionnaire de l'étendue de la pratique infirmière (QÉPI) afin de mesurer l'ÉEPI. Celui-ci a été élaboré en 2012 par D'Amour et al. à partir d'une analyse de la littérature pertinente, la consultation d'experts et les tests de validité dans le but de la mesurer. Le QÉPI présente 26 questions de type Likert notées sur une échelle de 6 (1, jamais; 2, rarement; 3, parfois; 4, fréquemment; 5 , presque toujours, 6, toujours) réparties en six dimensions de l'étendue de la pratique infirmière soit : 1- L'évaluation et la planification des soins; 2- L'enseignement à la clientèle et aux familles; 3- La communication et la coordination des soins; 4L'intégration et l'encadrement du personnel; 5- L'optimisation de la qualité et de la sécurité des soins; et 6- La mise à jour et l'utilisation des connaissances. Des niveaux de complexité des activités sont présents au sein de chaque dimension. Le niveau 1 (faible complexité) qui réfère au niveau de pratique de base attendu par les infirmières, y compris les novices. Le niveau 2 (complexité modérée) fait référence à des interventions plus complexes pouvant être effectuées par toutes les infirmières expérimentées, quelle que soit leur formation. Les éléments de niveau 3 (complexité élevée) sont des interventions nécessitant une formation de niveau universitaire ainsi qu'un bon niveau d'expérience. Les dimensions, items et niveaux de complexité des items du QÉPI sont présentés à l'appendice II.

La première version du QÉPI ou Actual scope of nursing practice (ASCOP) en anglais, a été testé auprès de 285 infirmières dans 22 unités de soins (médecine) de 11 différents types d'établissements au Canada dans une étude employant un devis mixte afin de mesurer l'étendue effective de la pratique d'infirmières en milieu hospitalier sur une 
unité de médecine-chirurgie en zone urbaine. Cette étude appelée le «Projet modèle d'utilisation des ressources infirmières » a été menée par D'Amour et al. en 2008 dans le but d'établir l'étendue de pratique infirmière au Québec. La collecte de données s'est effectuée par 66 entrevues, 22 groupes de discussion, 282 QÉPI (taux de réponse de 55\%), collecte de données administratives et recensement des patients. Cette étude a démontré un sous-déploiement des rôles infirmiers dans les unités de médecine avec un score moyen au QÉPI de 3,47 (les scores par dimensions variaient de 3,03 à 4,19).

Le QÉPI vise un score de 6. L'obtention de ce score est probablement utopique, mais les études consultées n'établissent pas de barème d'interprétation des scores obtenus. Dans l'étude de D'Amour et al. (2012), les dimensions dont les activités ont été le plus souvent mises en œuvre étaient l'évaluation et la planification des soins $(4,19)$ et l'enseignement au patient et à sa famille $(3,88)$. Les activités les moins souvent réalisées étaient l'intégration et la supervision du personnel $(3,03)$, la qualité des soins et la sécurité des patients $(3,11)$, ainsi que la mise à jour et l'utilisation des connaissances $(3,09)$. Ces résultats amènent les auteurs à proposer quatre recommandations basées sur leurs résultats et la revue de littérature afin d'élargir l'ÉPI : 1- Effectuer des modifications de l'environnement de pratique en lien avec les facteurs influençant l'ÉPI ; 2- Adopter des modèles de soins mettant l'accent sur les fonctions essentielles des infirmières ; 3- Revoir les structures de travail et les descriptions de poste ; 4- Offrir des occasions de développement au personnel (D’Amour et al., 2012). Le QÉPI a ensuite été utilisé dans plusieurs autres contextes présentés ici et résumés au tableau 1. 
Tableau 1 : Comparaison des résultats de l'ÉEPI ayant utilisé le QEPI ou ses dérivés

\begin{tabular}{|c|c|c|c|c|c|c|c|c|c|c|c|c|}
\hline \multirow[b]{2}{*}{$\begin{array}{c}\text { Auteurs } \\
\text { (Année) } \\
\text { Questionnaire } \\
\text { utilisé }\end{array}$} & \multirow[b]{2}{*}{$\begin{array}{l}\text { Domaine } \\
\text { (Lieu) } \\
\text { Contexte }\end{array}$} & \multirow[b]{2}{*}{$\mathrm{n}$} & \multirow[b]{2}{*}{$\begin{array}{l}\text { Score } \\
\text { global }\end{array}$} & \multicolumn{6}{|c|}{ Dimensions } & \multicolumn{3}{|c|}{$\begin{array}{l}\text { Niveaux de } \\
\text { complexité }\end{array}$} \\
\hline & & & & $\begin{array}{l}1 \\
\text { Évaluation et } \\
\text { planification } \\
\text { des soins }\end{array}$ & $\begin{array}{c}2 \\
\text { Enseignement } \\
\text { à la clientèle } \\
\text { et aux } \\
\text { familles }\end{array}$ & $\begin{array}{c}3 \\
\text { Communication } \\
\text { et coordination } \\
\text { des soins }\end{array}$ & $\begin{array}{c}4 \\
\text { Intégration et } \\
\text { encadrement } \\
\text { du personnel }\end{array}$ & $\begin{array}{c}5 \\
\text { Optimisation } \\
\text { de la qualité } \\
\text { et sécurité des } \\
\text { soins }\end{array}$ & $\begin{array}{l}6 \\
\text { Mise à jour et } \\
\text { utilisation des } \\
\text { connaissances }\end{array}$ & 1 & 2 & 3 \\
\hline $\begin{array}{c}\text { Younan et al. } \\
\quad(2019) \\
A-A S C O P\end{array}$ & $\begin{array}{l}\text { Hospitalier } \\
\text { (Liban) } \\
\text { Rural urbain }\end{array}$ & 2852 & 4,42 & 4,33 & 4,56 & 4,50 & 4,08 & 4,53 & 4,53 & & & \\
\hline $\begin{array}{c}\text { Braithwaite } \\
\text { (2016) } \\
A S C O P-P L\end{array}$ & $\begin{array}{c}\text { Services 1 }^{\text {re }} \\
\text { ligne } \\
\text { (Ontario) } \\
\text { Urbain }\end{array}$ & 181 & 4,81 & 4,83 & 5,16 & 4,86 & 4,20 & 4,66 & 5,12 & 5,31 & 4,76 & 4,49 \\
\hline $\begin{array}{c}\text { Lafleur } \\
(2016) \\
Q E P I\end{array}$ & $\begin{array}{l}\text { Santé } \\
\text { mentale } \\
\text { (Québec) } \\
\text { Urbain }\end{array}$ & 80 & 4,24 & 4,54 & 4,46 & 4,43 & 3,70 & 4,01 & 4,27 & 4,97 & 4,25 & 3,64 \\
\hline $\begin{array}{l}\text { Déry } \\
(2013) \\
Q E P I\end{array}$ & $\begin{array}{l}\text { Pédiatrie } \\
\text { (Québec) } \\
\text { Urbain }\end{array}$ & 301 & 3,21 & 3,95 & 3,79 & 3,01 & 2,64 & 2,84 & 2,89 & 4,24 & 3,34 & 2,26 \\
\hline $\begin{array}{c}\text { D'Amour et } \\
\text { al. }(2012) \\
Q E P I\end{array}$ & $\begin{array}{l}\text { Médecine- } \\
\text { chirurgie } \\
\text { (Québec) } \\
\text { Urbain }\end{array}$ & 285 & 3,47 & 4,2 & 3,5 & 3,4 & 3 & 3,1 & 2,8 & 4,39 & 3,71 & 2,54 \\
\hline
\end{tabular}


Le QÉPI a ensuite été utilisé en 2011 par Déry (2013), en milieu hospitalier sur une unité de pédiatrie en zone urbaine afin de mesurer l'ÉEPI, ses déterminants et son influence sur la satisfaction professionnelle. Des infirmières de cinq secteurs d'activités d'un centre hospitalier pédiatrique universitaire du Québec $(\mathrm{N}=301)$ ont répondu au QÉPI. Les résultats recueillis indiquent aussi une sous-utilisation $(3,21$; E.T. $=0,71)$ et identifient certaines caractéristiques du travail qui influencent le déploiement de l'étendue de la pratique infirmière et par le fait même la satisfaction professionnelle (Déry, 2013).

Ensuite, Lafleur (2016) a conduit une recherche en employant elle aussi le QÉPI afin de mesurer l'étendue de la pratique dans un Institut universitaire en santé mentale du Québec soit en milieu urbain auprès de 80 infirmières. Ses résultats démontrent un score plutôt satisfaisant de l'étendue de la pratique des infirmières $(4,24$; E.T.=0,63) en comparaison aux résultats de Déry (2013) et de D’amour et al. (2012).

De son côté, Braithwaite a conduit une étude en Ontario auprès des infirmières œuvrant dans les services de première ligne en zone urbaine en Ontario afin d'y mesurer l'étendue de la pratique. En premier lieu, une revue de littérature a permis l'adaptation du QÉPI au contexte de première ligne ontarien (version anglaise). Dans un deuxième temps, une étude transversale a permis de mesurer l'étendue de la pratique auprès de 178 infirmières autorisées au sein des organisations de soins primaires en Ontario. Les résultats présentent un score moyen de 4,81 (Braithwaite, 2016), ce qui est le score global, soit le plus élevé parmi les études recensées au Québec et ayant utilisé le QÉPI (Lafleur, 2016; Déry, 2013; D’Amour et al., 2012).

En 2018, le QÉPI a été traduit, adapté et validé en arabe par Fares, Clinton \& Younan (2018) pour utilisation au Liban dans la région de la Méditerranée orientale à l'aide des directives de l'Organisation mondiale de la santé (n.d.) et de l'Institut Mapi (2016) relatives à la traduction et à la validation culturelle des instruments. Le coefficient alpha de Cronbach pour les 26 items était de 0,96, ce qui lui confère une excellente cohérence interne. Les coefficients alpha de Cronbach de la sous-échelle vont de 0,71 
(mise à jour et utilisation des connaissances) à 0,84 (sécurité des patients et qualité des soins) (Fares et al., 2018). Par la suite, en 2019, une enquête descriptive transversale nationale de 2852 membres du personnel infirmier travaillant dans 39 hôpitaux au Liban a été menée soit en milieu hospitalier de zones rurales et urbaines à l'aide de cette version adaptée du QÉPI afin de mesurer l'étendue effective de la pratique par qualifications professionnelles (Younan, Clinton, Fares \& Samaha, 2019). L'échantillon final était composé de 2852 répondants. Le score moyen global était de 4,42 (4,09 à 4,56). Les activités les plus fréquemment pratiquées étaient celles liées à l'enseignement aux patients et leurs familles $(4,56)$, suivies de celles relatives à la qualité des soins et à la sécurité des patients $(4,53)$, à la mise à jour et à l'utilisation des connaissances $(4,35)$, et à la communication et à la coordination $(4,50)$. Les activités les moins exécutées étaient celles liées à l'évaluation et à la planification des soins $(4,32)$ et à l'intégration et à la supervision du personnel $(4,09)$.

En somme, les résultats démontrent une sous-utilisation de la main-d'œuvre infirmière et un déploiement sous-optimal de la pratique infirmière dans les hôpitaux du Québec, au Canada et ailleurs dans le monde (White \& al., 2008; Oelke \& al., 2008; D’Amour et al., 2012; Déry, 2013; Lafleur, 2016). Ceci toucherait également les soins de proximité en Ontario (Braithwaite, 2016). La plupart des études recensées ont toutefois été conduites en milieu hospitalier dans les grands centres urbains. Aucune ne dresse toutefois ce portait pour les services de proximité ou de première ligne en région éloignée. 


\subsection{BENEFICES DE LA PLEINE ETENDUE DE LA PRATIQUE DES INFIRMIERES EN SOINS PRIMAIRES}

Les bénéfices d'une pratique infirmière «étendue » dans les soins primaires sont nombreux. Des études démontrent que l'ajout d'infirmières autorisées exerçant pleinement leur rôle contribue à des retombées positives pour les soins de santé, l'organisation et les usagers. La pratique infirmière étendue serait aussi associée à des avantages pour les infirmières elles-mêmes qui sont présentés par la suite.

\subsubsection{Bénéfices pour les soins de santé, l'organisation et les usagers}

La littérature démontre généralement bien les effets bénéfiques d'une pratique infirmière étendue à l'égard de la qualité des soins que l'Organisation mondiale de la Santé (OMS) définit comme :

Une démarche qui doit permettre de garantir à chaque patient la combinaison d'actes diagnostiques et thérapeutiques qui lui assurera le meilleur résultat en termes de santé, conformément à l'état actuel de la science médicale, au meilleur coût pour un même résultat, au moindre risque iatrogène et pour sa plus grande satisfaction en termes de procédures, de résultats et de contacts humains à l'intérieur du système de soins (OMS, non-daté dans Secrétariat international des infirmières et infirmiers de l'espace francophone, 2015, page 8).

En ce sens, une revue de littérature a été réalisée par Parkinson et Parker et publiée en 2013 afin de préciser les rôles joués par les infirmières dans les soins de santé primaires pour assurer le suivi des personnes atteintes de maladies chroniques et complexes. Les 97 articles retenus portaient sur différentes interventions, dont la consultation, la surveillance, la prévention et l'éducation à la santé, la comparaison entre les soins dispensés par un médecin et ceux offerts par une infirmière ainsi que la formation des infirmières. Leur revue a démontré que les soins infirmiers constituent des soins de santé primaires offrant un bon rapport qualité-coût-efficacité. Les auteurs précisent que: «il existe un consensus 
international quant au rôle important que l'infirmière peut jouer pour offrir des soins de santé primaires appropriés, rentables et de grande qualité aux personnes vieillissantes atteintes de maladies chroniques et complexes » [traduction libre] (Parkinson \& Parker, 2013, p.588). Ensuite, une étude menée par Smolowitz et al. (2015) employant un devis qualitatif a étudié le rôle des infirmières en soins de santé primaires auprès de 16 dispensateurs de soins de santé primaires identifiés comme des modèles exemplaires à travers les États-Unis. Les participants rencontrés dans le cadre d'entrevues ont indiqué que l'ajout d'infirmières pratiquant à leur pleine étendue dans une équipe de soins de santé primaires permettrait une meilleure qualité des soins et des économies. Ils indiquent également qu'il y a un large intérêt pour une refonte des modèles de pratique de soins de santé primaires dans le but d'améliorer l'accès aux soins de santé de qualité et que les infirmières sont bien placées pour dispenser les soins et assumer leur rôle de leader clinique en raison de leur compréhension des priorités des usagers, de la famille et du système (Smolowitz et al., 2015). Jacobson (2012) arrive à des constats similaires dans le cadre d'une revue de la littérature dont l'objectif était de mettre en évidence l'efficacité des coûts et des résultats des équipes interprofessionnelles ou dirigées par des infirmières. Cette revue a mis l'accent sur la contribution des infirmières et des infirmières auxiliaires en tant que composantes essentielles des équipes interprofessionnelles dans la prise en charge des personnes atteintes de maladies chroniques complexes en soins primaires. L'auteur a notamment constaté qu'une utilisation accrue des ressources infirmières pouvait améliorer les contacts entre le système de santé et la clientèle, l'éducation aux usagers et à leur famille et la gestion de la maladie. De plus, le recours à des équipes interprofessionnelles offre un potentiel d'amélioration de l'accès aux soins de santé et des résultats positifs en matière de santé (Jacobson, 2012).

Une étude qualitative visant à évaluer la satisfaction de la clientèle envers les infirmières de pratique générale en Nouvelle-Zélande a été réalisée par Halcomb, Peters et Davies (2013). Dix-huit (18) entretiens téléphoniques semi-structurés ont été conduits et ont permis de démontrer que les usagers sont très satisfaits de la prestation des services 
de soins infirmiers en pratique générale et que celle-ci permet d'accroître l'accessibilité, et ce, au moindre coût financier pour les usagers (Halcomb et al., 2013).

Ensuite, une revue intégrative de 59 articles publiés entre 1983 et 2013 a permis d'identifier les caractéristiques principales des services de santé primaires dirigés par des infirmières (Holt, Zabler \& Baisch, 2014). Plusieurs caractéristiques sont ressorties soit : la satisfaction de la clientèle et des familles pour les soins et le système, un accès accru aux services de soins de santé, une rentabilité, des soins de santé de qualité ainsi que la satisfaction au travail des infirmières (Holt, et al., 2014). Randall, Crawford, Currie, River \& Betihavas (2017) ont également réalisé une revue systématique visant à identifier l'impact des cliniques communautaires dirigées par des infirmières sur l'état de santé et la satisfaction de la clientèle ainsi que sur l'accessibilité et la rentabilité. La revue comprenait au final 15 études pour un total de 3965 participants et les données étaient principalement autodéclarées. Les résultats indiquent que les cliniques dirigées par des infirmières ont un impact positif sur l'état de santé de la clientèle tels que l'adoption de saines habitudes de vie, l'amélioration de la gestion des symptômes ou de la santé (déclarée par les patients) ou l'amélioration de certains résultats cliniques objectifs comme le temps de guérison des ulcères, l'amélioration des scores d'écart type de l'indice de masse corporelle chez les enfants obèses et le régime de traitement des infections des voies respiratoires supérieures. Des impacts positifs ont également été recensés en ce qui concerne la satisfaction de la clientèle et l'accès aux soins. Les résultats sont toutefois mitigés quant au rapport coût-efficacité puisque les deux études que Randall et al. (2017) ont recensé sur ce sujet présentent des résultats différents. La première n'a signalé aucune différence statistique dans l'utilisation et le coût des ressources entre la prestation d'un service médical de clinique à domicile et une clinique dirigée par du personnel infirmier (Harrison et al., 2008 dans Randall et al., 2017) tandis que la deuxième a identifié des économies de coûts pour la clinique infirmière et le patient, pour un retour sur investissement de 34 \$ par 1 \$ investi (Bicki et al., 2013 dans Randall et al., 2017). 
Finalement, une analyse transversale a été réalisée au Québec afin d'évaluer l'expérience des patients concernant le rôle d'infirmières en matière de soins primaires (Borgès Da Silva, Brault, Pineault, Chouinard, Prud'homme, D’Amour et al., 2018). L’objectif était de vérifier l'hypothèse selon laquelle, dans les organisations de soins de santé primaires où les patients sont systématiquement suivis par une infirmière et où les compétences infirmières sont utilisées de manière optimale, l'expérience des patients en matière de soins est meilleure. Cette étude transversale constituait une analyse secondaire des données issues d'une enquête d'envergure menée en 2005 évaluant la performance des soins primaires en ce qui a trait à l'expérience des patients dans les deux régions les plus peuplées du Québec soit Montréal et la Montérégie (Lévesque, Pineault, Provost, Tousignant, Couture, Da Silva et Breton, 2010). Une enquête téléphonique destinée à la population $(n=1980)$ et une enquête postale s'adressant aux organisations $(n=606)$ ont été réalisées en 2005. Les résultats ont indiqué que le suivi systématique des patients par les infirmières améliorait l'expérience des patients en matière d'accessibilité (Borgès Da Silva et al., 2018).

\subsubsection{Bénéfices pour les infirmières}

Des études ont souligné qu'une meilleure utilisation des ressources infirmières par la pleine étendue de leur pratique contribuerait aussi à un accroissement de leur satisfaction professionnelle et de leur satisfaction au travail (Déry, 2013; Halcomb et Ashley, 2016).

L'étude de Déry (2013) visait à mesurer l'étendue effective de la pratique infirmière (ÉEPI) en pédiatrie hospitalière, son effet sur la satisfaction professionnelle et l'influence de certaines caractéristiques en utilisant un devis transversal corrélationnel. La satisfaction professionnelle des infirmières est une composante de la satisfaction au travail (Hinshaw et Atwood, 1985 cités dans Déry, 2013) et fait référence à « l'opinion du 
personnel infirmier sur la qualité des soins fournis, le temps nécessaire pour mener ses activités en matière de soins et le plaisir général de son rôle » (Hinshaw, Smeltzer et Atwood, 1987 cités dans Déry, 2013, p.10). Cette enquête a été réalisée auprès de 301 infirmières de la région de Montréal au Québec en utilisant des questionnaires autoadministrés (taux de réponse de $90 \%$ ). Les résultats ont démontré qu'un score plus élevé de l'ÉEPI mesuré à l'aide du QÉPI était associé à une influence positive directe sur la satisfaction professionnelle $(\beta=.118 ; p<0,01)$. Une autre étude d'Halcomb et Ashley (2016), visait à identifier les aspects les plus et les moins satisfaisants du travail des infirmières au sein des services de soins primaires. Cette dernière employant un devis exploratoire descriptif est arrivée à un constat similaire à celui de Déry (2013). Un sondage en ligne a été rempli par 1166 infirmières employées dans des établissements de soins de santé primaires en Australie (Halcomb et Asley, 2016). Les services de soins de santé primaires y étaient présentés comme un lieu où les soins infirmiers sont dispensés hors des limites d'un établissement de soins de courte durée comme des cabinets de médecine générale, des écoles/universités, des établissements correctionnels, des cliniques de santé sexuelle et des services de soins infirmiers communautaires. Le sondage présentait un seul élément quantitatif qui demandait aux participants d'évaluer leur degré de satisfaction à l'égard de 11 aspects de leur situation actuelle sur une échelle de Likert de cinq points (très insatisfaisant à très satisfaisant) et deux questions ouvertes où les participants devaient identifier les aspects les moins satisfaisants de leur travail. Les répondants ont identifié qu'une faible étendue de pratique constitue une raison d'insatisfaction vis-à-vis leur travail. «Je pense que nous pourrions faire beaucoup plus si nous n'étions pas aussi limités dans ce que nous faisons et dans comment nous le faisons» [traduction libre] (Halcomb et Asley, 2016, p. 542). Les répondants ont parlé de tâches non infirmières comme changer les rideaux des salles d'examen, nettoyer les tables d'examens et les armoires, s'assurer que les médecins disposent de papier dans leurs imprimantes, ramasser leurs ordures/linges au sol, vider les poubelles comme des corvées qui les empêchaient d'utiliser leurs compétences infirmières et d'effectuer les tâches infirmières pour lesquelles ils détiennent la formation et l'expérience (Halcomb et Ashley, 2016) telles que 
l'évaluation de la condition physique et mentale des usagers, la planification et la coordination des soins, l'enseignement et le suivi de la clientèle.

En somme, selon les études ayant traité des bénéfices et effets associés à une pratique infirmière « étendue », dont l'une réalisée au Québec, il parait souhaitable que les systèmes de santé misent davantage sur la profession infirmière pour la dispensation des services de proximité compte tenu la nature et l'ampleur de ses retombées sur l'accessibilité aux soins et services de santé. Ultimement, le patient, les infirmières et le système de santé profiteraient d'une meilleure utilisation des ressources infirmières. Celle-ci contribuerait à un accroissement de l'efficience et de la performance des organisations en permettant une économie des coûts de fonctionnement (Parkinson et Parker, 2013; Smolowitz et al., 2015), une plus grande accessibilité aux soins et services de santé (Jacobson, 2012 ; Borgès Da Silva, 2018) ainsi qu'une qualité et sécurité des soins accrue (Houweling et al., 2011; Jacobson, 2012 ; Parkinson et Parker, 2013 ; Aroke, 2014; Halcomb, Peters et Davies, 2013). Des études ont également démontré qu'une meilleure utilisation des ressources infirmières contribuerait à un accroissement de la satisfaction de la clientèle (Parkinson et Parker, 2013; Houweling et al., 2011; Jacobson, 2012; Borgès Da Silva et al., 2018) ainsi qu'à une plus grande satisfaction professionnelle (Déry, 2013; Halcomb et Ashley, 2016).

\subsection{CARACTERISTIQUES INFLUENÇANT L'ETENDUE DE LA PRATIQUE INFIRMIERE ET STRATEGIES POUR L'ACCROITRE}

Cette section présente les différentes études qui ont mis en lumière des caractéristiques influençant l'ÉPI et se termine par un tableau intégrateur de l'ensemble des caractéristiques mises en évidence dans ces études (voir Tableau 2).

Tel que présenté précédemment dans la section 2.2, Besner et al., (2005) ont mené une étude sur la perception des infirmières par rapport à l'étendue de leur pratique et aux 
facteurs l'influençant. Pour ce faire, des entrevues sur la vision de l'ÉPI ont permis d'identifier certaines caractéristiques d'influence. Les auteurs ont mis en évidence : des caractéristiques individuelles, telles que la formation, les connaissances et les compétences; des caractéristiques professionnelles, telles que les relations professionnelles, l'environnement de travail, le chevauchement et l'ambiguïté des rôles ; des caractéristiques organisationnelles et contextuelles telles que les besoins de la clientèle, la charge de travail, la disponibilité des ressources et le soutien du gestionnaire (Besner et al., 2005).

En 2008, Oelke et al. ont publié les résultats d'une étude visant à identifier les facilitateurs et barrières à l'étendue de la pratique infirmière en employant un devis mixte. Les données ont été recueillies sur 14 unités de soins de courte durée (médecine, chirurgie, soins intensifs, réadaptation et psychiatrie) dans trois régions sanitaires auprès de différents prestataires de soins infirmiers. Un total de 236 entrevues ont été réalisées : 167 auprès d'infirmières (31 infirmières auxiliaires, 85 infirmières et 11 infirmières en santé mentale) et 40 auprès de gestionnaires infirmiers et autres. Les facilitateurs identifiés sont: le travail en équipe, le soutien et le leadership du gestionnaire ainsi que le soutien à l'éducation et au maintien des compétences. Les barrières identifiées étaient quant à elles associées à la difficulté à décrire l'étendue de la pratique, la surcharge de travail, la complexité des soins, le manque de temps, la communication inefficace et l'incapacité à travailler en équipe. Ces auteurs recommandent également de : «mener des recherches plus approfondies dans les milieux non urbains ainsi que dans les secteurs communautaires afin de déterminer les éventuelles différences » (Oelke, 2008, p.69).

Les résultats de l'étude de Lampron visant à décrire l'ÉPI et identifier les facteurs organisationnels l'influençant ont aussi permis d'identifier des facteurs organisationnels influençant l'étendue de la pratique soit : la charge de travail, l'environnement de travail ainsi que la composition et les caractéristiques des équipes de soins (Lampron, 2010). 
Dans le cadre de ses travaux, Déry (2013) a participé à la création du modèle de l'étendue actuelle de la pratique infirmière « Actual SCOpe of nursing Practice model (ASCOP) ». Ce modèle prend appui sur la théorie des caractéristiques de l'emploi (Hackman et Oldham, 1974, cité dans Déry, 2013), sur le modèle tensionautonomie (Karasek, 1985, cité dans Déry, 2013), sur la théorie du rôle (Biddle, 1979, cité dans Déry, 2013) et sur les travaux de D'Amour et al. (2012) portant sur ÉPI. Le modèle met aussi en relation des caractéristiques du travail et des caractéristiques individuelles d'influence potentielle sur le déploiement de la pratique infirmière. Les caractéristiques du travail étant présentées comme influençant l'ÉPI sont la latitude décisionnelle, les demandes psychologiques, l'ambiguïté de rôle, les conflits de rôle et la surcharge de rôle. Les caractéristiques individuelles des infirmières sont, quant à elles, l'âge, le niveau de formation, l'expérience et le besoin de croissance individuelle de l'infirmière. Le modèle du SCOP prévoit également un lien entre l'étendue de la pratique infirmière et la satisfaction professionnelle.

L'analyse du concept de plein potentiel infirmier (Aroke, 2014) a mené au développement d'un modèle présentant les caractéristiques contribuant au déploiement du plein potentiel infirmier en trois grandes catégories soit : 1- le champ d'exercices (lois et règlements de l'État, politiques institutionnelles), 2- les facteurs environnementaux (type d'installation, besoin du patient, moyen, besoin et leadership, disponibilité de l'effectif) et 3- les traits personnels (éducation, expérience, motivation et objectifs) (Aroke, 2014).

Également, entre 2005 et 2007, Oelke, Besner et Carter (2014) ont réalisé une étude décrivant le rôle actuel des infirmières dans trois réseaux de soins primaires dans le Canada occidental et identifiant les opportunités d'utilisation optimale des infirmières dans ces milieux. Pour ce faire, cette étude a été réalisée en deux phases, soit une première phase durant laquelle des entretiens semi-structurés ont été menés auprès de 42 personnes (infirmières, autres prestataires de services, médecins, décideurs) dans l'ensemble des réseaux de soins primaires ainsi que l'examen documentaire des descriptions de poste en soins pour mieux comprendre les tâches de suivi en cours dans les réseaux participants. 
Des méthodes mixtes (entrevues et revue de littérature) ont été utilisées lors de la seconde phase. Le manque de collaboration entre les infirmières et d'autres fournisseurs, en particulier des médecins, ont eu une incidence négative sur la définition du rôle d'infirmière. Parmi les autres obstacles à la mise en place de rôles figuraient la rémunération à l'acte des médecins, les structures et processus de gestion, le manque d'accès aux dossiers médicaux électroniques et le manque d'expérience en ce qui concerne l'éducation aux soins de santé primaires dans le cadre de la pratique (Oelke et al., 2014).

Une étude exploratoire des écrits, conduite pour le compte de l'Association des infirmières et infirmiers du Canada (AIIC), a aussi permis de présenter plusieurs obstacles structurels et liés aux processus qui empêchent les infirmières d'occuper leur champ de pratique de façon optimale dans les contextes de soins primaires ainsi que des stratégies et recommandations afin d'optimiser le rôle des infirmières dans la prestation des services de soins primaires au Canada. Pour ce faire, 109 articles ont été retenus et des entrevues avec des informateurs clés ont également été réalisées. Les caractéristiques ont, par la suite, été classées en trois catégories, soit les caractéristiques individuelles (manque de connaissances et/ou de compétences et/ou d'expérience pour le rôle, manque de compréhension du champ d'exercices) les caractéristiques organisationnelles (médecins employeurs d'infirmières, manque de clarté des rôles, isolement des infirmières, manque de politiques pour appuyer le rôle infirmier et le travail d'équipe, absence de description de poste) et les caractéristiques dans les systèmes de santé (compréhension du champ d'exercice de la profession infirmière, restrictions législatives applicables au champ d'exercices, accès aux programmes de formations pertinentes, financement et modèles de financement (Association des infirmières et infirmiers du Canada, 2014).

Une enquête transversale nationale australienne sur le personnel infirmier en médecine générale dans un contexte de première ligne a été menée en 2003-2004 et en 2009-2010 afin de décrire les caractéristiques démographiques et d'emploi actuel des infirmières australiennes travaillant en pratique générale et explorer les raisons de leurs heures supplémentaires. Pour ce faire, une analyse secondaire a été réalisée à partir de 
deux enquêtes plus vastes à méthodes mixtes ayant porté sur les rôles cliniques de ces infirmières (Halcomb, Salamonson, Davidson, Kaur et Young, 2014). La première enquête portait sur 284 infirmières et la seconde sur 235 infirmières employées en pratique générale dans six États australiens. Des infirmières pratiquant dans des quartiers défavorisés et des cliniques éloignées participaient aux deux enquêtes. Les obstacles identifiés à l'élargissement du rôle des infirmières étaient les juridictions professionnelles, le chevauchement des rôles, l'adéquation du rôle actuel par rapport au plein déploiement du rôle, l'attitude négative des omnipraticiens envers l'élargissement du rôle des infirmières, le manque d'opportunité des infirmières d'élargir leur rôle, le manque de formation des infirmières, l'impossibilité de prescrire des médicaments, la perception des patients par rapport au rôle infirmier, le manque de compétence clinique, la description de l'emploi, le manque de confiance pour négocier avec les omnipraticiens et le manque de désir personnel des infirmières d'améliorer leur rôle. Les facilitateurs identifiés par les infirmières étaient leur accès à la formation, la collaboration avec les omnipraticiens, la possibilité, pour elles, de dispenser des soins de santé primaires, leur satisfaction au travail, leur contribution aux plans de gestion, la possibilité de façonner leur rôle pour répondre aux besoins locaux, leur autonomie de pratique, des retours positifs de la clientèle par rapport aux soins reçus, la nature nouvelle/passionnante du rôle, la gestion moins restrictive, les heures améliorées et de bonnes conditions d'emploi (Halcomb et al., 2014).

Une recension des écrits, réalisée par Roy (2015), afin d'identifier des caractéristiques influençant l'étendue de pratique infirmière ainsi que des stratégies pouvant favoriser son déploiement optimal en se basant sur le modèle de pratique professionnelle (MPP) du Massachusetts General Hospital (MGH) développé en 1996 et révisé en 2007 vient préciser certaines de ces composantes. L'analyse des écrits recensés a permis de documenter 26 caractéristiques pouvant influencer le déploiement de l'étendue de pratique des infirmières ainsi que 25 stratégies potentielles afin d'optimiser le plein déploiement de l'étendue de pratique des infirmières (Roy, 2015). Ces caractéristiques et stratégies ont été présentées pour l'ensemble des neuf composantes du 
modèle soit : 1- vision et valeurs; 2- standards de pratique; 3 - pratique réflexive; 4développement professionnel; 5- soins centrés sur le patient; 6- reconnaissance clinique et avancement; 7-gouvernance; 8- résultats probants et recherche; 9- innovation et travail d'équipe (Roy, 2015). Les 26 caractéristiques sont présentées dans le tableau 2.

Une autre étude, qui visait à examiner et à décrire les facilitateurs et les obstacles au champ d'exercices actuel au sein d'un échantillon national d'infirmières et de sagesfemmes en Irlande, a été conduite par Fealy, Rohde, Casey, Brady, Hegarty, Kennedy, McNamara, O'Reilly et Prizeman (2015). L'enquête a été réalisée à l'aide du Scope-Q, un questionnaire postal à auto-évaluation de 64 questions conçu pour l'étude. Le questionnaire mesurait les variables associées au champ d'activités actuel et contenait également une échelle de 19 obstacles, l'échelle Scope-Q-Barriers (Scope-QB), élaborée à partir d'une revue de la littérature. Le questionnaire a été prétesté sur un groupe de 16 étudiants en sciences infirmières diplômés qui entreprenaient un diplôme universitaire en sciences infirmières. Les analyses portent sur les questionnaires de 1010 infirmières pratiquant dans divers milieux autant hospitaliers que de première ligne. Les facilitateurs les plus fréquemment identifiés à l'étendue de la pratique ont été la satisfaction professionnelle et les besoins des patients, le soutien des collègues et de l'organisation ainsi que l'accès à une formation professionnelle continue. Les obstacles à l'utilisation pleine et entière du champ d'exercices des infirmières les plus fréquemment signalés étaient la résistance des autres disciplines de la santé, la peur des conséquences juridiques, les contraintes de temps et une rémunération insuffisante (Fealy et al. 2015).

Birks, Smithson, Lindsay et Davis (2018) ont eux aussi conduit une étude employant un devis descriptif transversal afin d'explorer les déterminants du champ de pratique d'un échantillon national d'infirmières australiennes inscrites à l'aide d'un sondage en ligne. Pour y parvenir, les auteurs ont repris le questionnaire Scope-Q, le questionnaire conçu par Fealy et al. (2015). L'outil a été révisé pour l'adapter au contexte infirmier australien (Scope-QAu), avec l'ajout de directives professionnelles pertinentes élaborées par Nursing and Midwifery Conseil de l'Australie. Le Scope-QAu comprend 
cinq catégories : 1- Mon champ de pratique; 2- Moyens et obstacles à un champ d'exercices élargi; 3- Ressources pour ma pratique professionnelle; 4-Mon rôle professionnel; et 5- Informations démographiques. Au total, 1874 questionnaires ont été soumis et $1231(65,7 \%)$ ont été suffisamment complétés pour être utiles à l'analyse. Les résultats révèlent que peu de caractéristiques démographiques prédisaient la façon dont les infirmières décidaient de leur propre champ de pratique. Les caractéristiques étudiées ont été : 1- l'expérience, 2- la formation et 3- la position géographique (ville, région, région éloignée...). L'étude a démontré que les infirmières autorisées ne se fient pas uniquement aux lignes directrices professionnelles ou aux cadres réglementaires pour déterminer leur champ d'application, mais également aux pairs et aux gestionnaires hiérarchiques (Birks et al., 2018).

Dans une revue de littérature publiée en 2015, Vancol-Fable a aussi identifié des caractéristiques organisationnelles susceptibles d'influencer l'étendue de la pratique telle que la formation, le soutien des gestionnaires, le manque de temps, la reconnaissance et le leadership transformationnel. Ces constats l'amènent à proposer de mettre en place un rôle d'infirmière clinicienne gestionnaire de cas au sein du centre ambulatoire pour optimiser l'étendue de la pratique dans ce milieu de première ligne (Vancol-Fable, 2015).

De leur côté, dans leur étude menée au Liban en milieu hospitalier de zones rurales et urbaines présenté précédemment (tableau 1), Younan, Clinton, Fares et Samaha (2019) n'ont pas établi de différences significatives dans les scores moyens globaux des activités infirmières réalisées entre les titulaires de diplômes universitaires et techniques qui auraient indiqué que la formation constitue un facteur d'influence (Younan et al. 2019). Les résultats de l'étude de Younan et al. (2019) ont également démontré que le nombre d'années d'expérience constitue une caractéristique d'influence positive de l'étendue effective de la pratique, mais pas la qualification professionnelle, car les scores totaux étaient similaires, peu importe cette qualification professionnelle. Les différents types de qualifications étudiés étaient les infirmières (détenant un diplôme de niveau lycée ou 
universitaire) ainsi que les infirmières auxiliaires (ne détenant aucun diplôme) (Younan et al. 2019).

Le tableau 2 présente les caractéristiques mises en lumière dans les différentes études présentées dans cette section et catégorisées selon les travaux menés par Besner et al. (2005) présenté plus tôt dans cette section. 
Tableau 2 : Caractéristiques influençant le déploiement de l'ÉPI selon les études

\begin{tabular}{|c|c|c|c|c|c|c|c|c|c|c|c|c|}
\hline & Auteurs & $\begin{array}{l}\text { Besner } \\
\text { et al. }\end{array}$ & $\begin{array}{l}\text { Oelke et } \\
\text { al. }\end{array}$ & Déry & Aroke & AllC & $\begin{array}{l}\text { Halcomb } \\
\text { et al. }\end{array}$ & Roy & $\begin{array}{l}\text { Vancol- } \\
\text { Fable }\end{array}$ & $\begin{array}{l}\text { Fealy et } \\
\text { al. }\end{array}$ & $\begin{array}{l}\text { Birks et } \\
\text { al. }\end{array}$ & $\begin{array}{l}\text { Younan } \\
\text { et al. }\end{array}$ \\
\hline & Année & 2005 & 2008 & 2013 & 2014 & 2014 & 2014 & 2015 & 2015 & 2015 & 2018 & 2019 \\
\hline & Devis & $\begin{array}{l}\text { Étude } \\
\text { qualit. }\end{array}$ & $\begin{array}{l}\text { Étude } \\
\text { quali }\end{array}$ & $\begin{array}{l}\text { Quanti. } \\
\text { Descript. }\end{array}$ & $\begin{array}{l}\text { Étude de } \\
\text { concept }\end{array}$ & $\begin{array}{l}\text { Revue } \\
\text { écrits }\end{array}$ & $\begin{array}{l}\text { Méthode } \\
\text { mixte }\end{array}$ & $\begin{array}{l}\text { Revue } \\
\text { écrits }\end{array}$ & $\begin{array}{l}\text { Revue } \\
\text { écrits }\end{array}$ & $\begin{array}{l}\text { Quanti. } \\
\text { Descript. }\end{array}$ & $\begin{array}{l}\text { Quanti. } \\
\text { Descript. }\end{array}$ & $\begin{array}{l}\text { Quanti } \\
\text { Descript. }\end{array}$ \\
\hline & Milieu & $\mathrm{CH}$ & $\mathrm{CH}$ & $\mathrm{CH}$ & Gen. & $1 \mathrm{~L}$ & $1 \mathrm{~L}$ & Gen. & Ambulat. & $\mathrm{CH}+1 \mathrm{~L}$ & $\mathrm{CH}+1 \mathrm{~L}$ & $\mathrm{CH}$ \\
\hline \multicolumn{13}{|c|}{ Catégories et caractéristiques } \\
\hline \multirow[t]{9}{*}{ Personnelles } & Capacité à décrire son ÉPI & $\mathrm{X}$ & & & & $\mathrm{X}$ & & $\mathrm{X}$ & & & & \\
\hline & $\begin{array}{l}\text { Perception et } \begin{array}{c}\text { conception } \\
\text { individuelles du déploiement } \\
\text { optimal de l'ÉPI }\end{array} \\
\end{array}$ & & & & & & & $\mathrm{x}$ & & & & \\
\hline & Expérience de travail & $\bar{X}$ & & $\mathrm{X}$ & & & & $\mathrm{X}$ & & & & $\mathrm{X}$ \\
\hline & Compétence & & & & & & $\mathrm{X}$ & & & & & \\
\hline & Formation & $\mathrm{X}$ & & $\mathrm{X}$ & $\mathrm{X}$ & $\mathrm{X}$ & $\mathrm{X}$ & $\mathrm{X}$ & $\mathrm{X}$ & & & \\
\hline & Satisfaction professionnelle & & & & & & $\mathrm{X}$ & & & $\mathrm{X}$ & & \\
\hline & $\begin{array}{l}\text { Désir personnel d'améliorer leur } \\
\text { rôle }\end{array}$ & & & & & & $\mathrm{X}$ & & & & & \\
\hline & $\begin{array}{l}\text { Besoin de croissance } \\
\text { personnelle }\end{array}$ & & & $\mathrm{x}$ & & & & & & & & \\
\hline & Âge & & & $X$ & & & & & & & & \\
\hline \multirow[t]{11}{*}{ Professionnelles } & Influence et de pouvoir politique & & & & & & & $\bar{X}$ & & & & \\
\hline & $\begin{array}{l}\text { Politiques et procédures des } \\
\text { organisations }\end{array}$ & & & & $\mathrm{X}$ & $\mathrm{x}$ & & $\mathrm{x}$ & & & $\mathrm{x}$ & \\
\hline & $\begin{array}{lll}\begin{array}{l}\text { Adoption } \\
\text { cliniques }\end{array} & \text { locale } & \text { d'outils } \\
\end{array}$ & & & & & & & $\mathrm{x}$ & & & & \\
\hline & $\begin{array}{l}\text { Niveau de reconnaissance de } \\
\text { l'infirmière comme partenaire à } \\
\text { part entière de l'équipe de soins }\end{array}$ & & & & & & $X$ & $\mathrm{x}$ & & $\mathrm{x}$ & & \\
\hline & $\begin{array}{l}\text { Littérature ou guidance sur la } \\
\text { mise en valeur du rôle }\end{array}$ & & & & & & & $\mathrm{x}$ & & & & \\
\hline & Connaissance du rôle infirmier & & & & & $\mathrm{X}$ & $\mathrm{X}$ & $\mathrm{X}$ & & & & \\
\hline & $\begin{array}{l}\text { Leadership et soutien des } \\
\text { gestionnaires et des infirmières }\end{array}$ & & $x$ & & $\mathrm{x}$ & $x$ & $x$ & $\mathrm{x}$ & $\mathrm{x}$ & $X$ & $X$ & \\
\hline & $\begin{array}{l}\text { Harmonisation de la littérature } \\
\text { quant à la description de } \\
\text { l'étendue de pratique }\end{array}$ & & & & & & & $X$ & & & & \\
\hline & $\begin{array}{l}\text { Juridictions professionnelles et } \\
\text { dynamiques de pouvoir }\end{array}$ & & & & $x$ & $X$ & $X$ & $X$ & & $X$ & & \\
\hline & $\begin{array}{l}\text { Négociation individuelle et ad } \\
\text { hoc du rôle et de l'étendue de } \\
\text { pratique }\end{array}$ & & & & & & $X$ & $X$ & & & & \\
\hline & Capacité de prescrire & & & & & & $\mathrm{X}$ & & & & & \\
\hline
\end{tabular}




\begin{tabular}{|c|c|c|c|c|c|c|c|c|c|c|c|c|}
\hline & Auteurs & $\begin{array}{l}\text { Besner } \\
\text { et al. }\end{array}$ & $\begin{array}{l}\text { Oelke et } \\
\text { al. }\end{array}$ & Déry & Aroke & AllC & $\begin{array}{l}\text { Halcomb } \\
\text { et al. }\end{array}$ & Roy & $\begin{array}{l}\text { Vancol- } \\
\text { Fable }\end{array}$ & $\begin{array}{l}\text { Fealy et } \\
\text { al. }\end{array}$ & $\begin{array}{l}\text { Birks et } \\
\text { al. }\end{array}$ & $\begin{array}{l}\text { Younan } \\
\text { et al. }\end{array}$ \\
\hline & Année & 2005 & 2008 & 2013 & 2014 & 2014 & 2014 & 2015 & 2015 & 2015 & 2018 & 2019 \\
\hline & Devis & $\begin{array}{l}\text { Étude } \\
\text { qualit. }\end{array}$ & $\begin{array}{l}\text { Étude } \\
\text { quali }\end{array}$ & $\begin{array}{l}\text { Quanti. } \\
\text { Descript. }\end{array}$ & $\begin{array}{l}\text { Étude de } \\
\text { concept }\end{array}$ & $\begin{array}{l}\text { Revue } \\
\text { écrits }\end{array}$ & $\begin{array}{l}\text { Méthode } \\
\text { mixte }\end{array}$ & $\begin{array}{l}\text { Revue } \\
\text { écrits }\end{array}$ & $\begin{array}{l}\text { Revue } \\
\text { écrits }\end{array}$ & $\begin{array}{l}\text { Quanti. } \\
\text { Descript. }\end{array}$ & $\begin{array}{l}\text { Quanti. } \\
\text { Descript. }\end{array}$ & $\begin{array}{c}\text { Quanti } \\
\text { Descript. }\end{array}$ \\
\hline & Milieu & $\mathrm{CH}$ & $\mathrm{CH}$ & $\mathrm{CH}$ & Gen. & $1 \mathrm{~L}$ & $1 \mathrm{~L}$ & Gen. & Ambulat. & $\mathrm{CH}+1 \mathrm{~L}$ & $\mathrm{CH}+1 \mathrm{~L}$ & $\mathrm{CH}$ \\
\hline \multicolumn{13}{|c|}{ Catégories et caractéristiques } \\
\hline \multirow[t]{15}{*}{ Organisationnelles } & $\begin{array}{l}\text { Réseautage et rétroaction par } \\
\text { les pairs }\end{array}$ & & & & & & & $\mathrm{x}$ & & & & \\
\hline & $\begin{array}{l}\text { Période consacrée } \\
\text { réflexion sur la pratique }\end{array}$ & & & & & & & $\mathrm{x}$ & & & & \\
\hline & $\begin{array}{l}\text { Types de modèles de prestation } \\
\text { de soins déployés dans les } \\
\text { milieux }\end{array}$ & & & & $x$ & & $\mathrm{x}$ & $\mathrm{X}$ & & & & \\
\hline & $\begin{array}{l}\text { Reconnaissance et valorisation } \\
\text { du rôle infirmier }\end{array}$ & & & & & & $\mathrm{x}$ & $x$ & $x$ & & & \\
\hline & Utilisation des ressources & & & & $\mathrm{X}$ & & & $\mathrm{X}$ & & & & \\
\hline & Interprétation des rôles & & & $\mathrm{X}$ & & $\mathrm{X}$ & $\mathrm{X}$ & $\mathrm{X}$ & & & & \\
\hline & $\begin{array}{lcc}\begin{array}{l}\text { Collaboration } \\
\text { différents professionnels }\end{array} & \text { les } \\
\end{array}$ & & $x$ & & & $\mathrm{x}$ & $\mathrm{x}$ & $\mathrm{x}$ & & & & \\
\hline & Communication & & $\mathrm{X}$ & & & & & $\mathrm{X}$ & & & & \\
\hline & Confiance & & & & & & $\mathrm{X}$ & $\mathrm{X}$ & & & & \\
\hline & Modalités de financement & & & & & $\mathrm{X}$ & & & & $\mathrm{X}$ & & \\
\hline & Charge de travail & & $\mathrm{X}$ & $\mathrm{X}$ & & & & & $\mathrm{X}$ & $\mathrm{X}$ & & \\
\hline & Condition d'emploi & & & & & & $\mathrm{X}$ & & & & & \\
\hline & Leadership transformationnel & & & & & & & & $\mathrm{X}$ & & & \\
\hline & Autonomie & & & $\mathrm{X}$ & & & & & & & & \\
\hline & Demande psychologique & & & $\mathrm{X}$ & & & & & & & & \\
\hline \multirow[t]{3}{*}{ Contextuelles } & $\begin{array}{l}\text { Accès à la formation initiale et } \\
\text { continue }\end{array}$ & & $\mathrm{x}$ & & & & $\mathrm{x}$ & $\mathrm{x}$ & & $\mathrm{x}$ & & \\
\hline & $\begin{array}{l}\text { Accès à des programmes de } \\
\text { mentorat }\end{array}$ & & & & & & & $X$ & & & & \\
\hline & $\begin{array}{l}\text { Acuité et la complexité des } \\
\text { besoins de soins des patients }\end{array}$ & & $\mathrm{x}$ & & $x$ & & & $X$ & & $x$ & & \\
\hline
\end{tabular}


En somme, la littérature démontre une panoplie d'effets bénéfiques d'une pratique infirmière étendue sur la qualité des soins de santé, l'organisation des services, les usagers et les infirmières elles-mêmes. Globalement, l'ÉPI contribuerait à la qualité des soins. Malgré ces bénéfices, les études démontrent un sous-déploiement de la pratique infirmière. Plusieurs études poursuivant l'objectif d'en mesurer le déploiement ont employé le QÉPI afin de mesurer l'ÉEPI. Également, la littérature présente un grand nombre de caractéristiques influençant l'ÉPI qu'il est possible de regrouper en différentes catégories, soit les caractéristiques individuelles, professionnelles, organisationnelles et contextuelles. Finalement, le choix du milieu de cette étude se voudra cohérent avec les recommandations issues de la recherche, soit de conduire des recherches dans les milieux non urbains ainsi que dans les secteurs non hospitaliers (Besner et al., 2005; Oelke et al., 2008). Celle-ci tentera de déterminer si des différences existent avec les études qui ont précédemment été réalisées en milieux urbains et en secteur hospitalier et de documenter la situation dans les milieux éloignés.

\subsection{OBJECTIFS DE LA RECHERCHE}

Ainsi, à la lumière de la problématique présentée et de l'état des connaissances réalisé, le but de cette recherche est d'établir un portrait de l'étendue de la pratique des infirmières travaillant dans les services de proximité dans une région éloignée du Québec.

Les objectifs de recherche seront donc de :

1- Mesurer l'étendue de la pratique infirmière dans les services de proximité de la région éloignée à l'étude.

2- Identifier les caractéristiques individuelles, professionnelles, organisationnelles et contextuelles influençant cette étendue dans le contexte des services de proximité de la région éloignée à l'étude. 


\section{CHAPITRE 3 \\ CADRE DE RÉFÉRENCE}

La première section de ce chapitre présente les définitions retenues des concepts à l'étude. La seconde propose une schématisation illustrant la mise en relation des concepts.

\subsection{CONCEPTS A L'ETUde}

Les concepts à l'étude sont l'étendue de la pratique, les régions éloignées, les services de proximité et la pratique infirmière dans ces services ainsi que les caractéristiques influençant l'étendue de la pratique.

\subsubsection{L'étendue de la pratique infirmière}

Dans le cadre de cette étude, la définition retenue pour l'étendue de la pratique infirmière est celle de l'étendue effective de la pratique infirmière (EEPI) de D'Amour et al. (2012) qui la présente comme l'éventail des fonctions et responsabilités déployées par l'infirmière en lien avec ses compétences, son expérience et son champ d'exercices. Cette définition est retenue, car elle englobe l'ensemble des autres définitions citées dans ce mémoire au chapitre 2 .

\subsubsection{Les régions éloignées}

Cette EEPI est étudiée dans les contextes des régions éloignées du Québec et des services de proximité. Dans cette étude, la conception de Gauthier et al. (2009) est adoptée 
pour définir une région éloignée sous l'angle de l'organisation des soins et services de santé comme une région située à l'extérieur des noyaux urbains et leur périphérie (champs d'influence) qu'ils situent à $250 \mathrm{~km}$. Au Québec, ces champs d'influence correspondent à Québec et Montréal où l'on trouve toute la gamme de services de santé.

\subsubsection{Les soins et services de proximité}

Les soins et services de proximité référent, dans cette étude, aux soins de santé primaire que 1'Association des infirmières et infirmiers du Canada (AIIC) (2014) définit comme l'éventail des modèles de soins de santé de première ligne qui englobent la promotion de la santé, le développement communautaire et la collaboration intersectorielle pour s'attaquer aux déterminants sociaux de la santé (Aggarwal et Hutchison, 2012). L'AIIC soutient qu'il existe plusieurs modèles de prestation des soins primaires, dont les centres de santé communautaire, les groupes de médecine familiale et les unités de médecine familiale. Dans ce mémoire réalisé au Québec, les services de proximité désignent l'ensemble des services de première ligne offerts par les CLSC dans le cadre de leurs programmes de services communautaires (services de santé publique, périnatalité, soutien à domicile, soins de santé courants, suivi d'intensité variable en santé mentale), dans les groupes de médecine familiale et les groupes de médecine familiale universitaires ainsi que dans les points de services CLSC communément appelés dispensaires.

\subsubsection{La pratique infirmière dans les services de proximité}

Chaque profession est définie par un champ d'exercices qui la décrit de façon générale en faisant ressortir la nature et la finalité de sa pratique ainsi que ses principales activités. Le champ d'exercices est régi par la Loi sur les infirmières et les infirmiers qui 
établit le domaine de pratique (la marque distinctive de la profession) et balise le contexte d'application des activités réservées aux membres de l'ordre professionnel. (OIIQ, 2016a). Au fil des ans, l'évolution des besoins et des pratiques en santé a obligé sa modernisation par la redéfinition des champs d'exercices de plusieurs professionnels de la santé. C'est donc le 30 janvier 2003 qu'est entrée en vigueur la grande majorité des dispositions générales de la Loi modifiant le Code des professions et d'autres dispositions législatives dans le domaine de la santé. Celles-ci ont amené une redéfinition du champ d'exercices des infirmières confirmant la reconnaissance par le législateur de leur compétence et de leur responsabilité à l'égard de l'évaluation clinique. Ainsi, l'article 36 de la Loi sur les infirmières et les infirmiers définit depuis le champ d'exercices infirmier comme :

L'exercice infirmier consiste à évaluer l'état de santé, à déterminer et à assurer la réalisation du plan de soins et de traitements infirmiers, à prodiguer les soins et les traitements infirmiers et médicaux dans le but de maintenir et de rétablir la santé de l'être humain en interaction avec son environnement et de prévenir la maladie ainsi qu'à fournir les soins palliatifs (Loi sur les infirmières et les infirmiers, article 36).

Tel que stipulé dans le champ d'exercices, l'infirmière a l'obligation légale de réaliser un plan de soins et de traitements infirmiers plus communément appelé «plan thérapeutique infirmier ». Celui-ci est :

Déterminé et ajusté par l'infirmière à partir de son évaluation clinique et consignée au dossier du client, le plan thérapeutique infirmier dresse le profil clinique évolutif des problèmes et des besoins prioritaires du client. Il fait également état des directives infirmières données en vue d'assurer le suivi clinique du client et qui portent notamment sur la surveillance clinique, les soins et les traitements. Couvrant le continuum de soins et de services, le plan thérapeutique infirmier peut englober un ou plusieurs épisodes de soins (OIIQ, 2006, page 5).

Dans le cadre de l'exercice infirmier, 17 activités sont réservées à l'infirmière et à l'infirmier. Celles-ci sont présentées à l'appendice II pour le secteur des services de proximité. 
Le Québec est la seule province canadienne à octroyer le permis d'exercice à la suite de l'obtention du diplôme collégial (postsecondaire) en soins infirmiers. Ainsi, au Québec, deux niveaux de formation mènent à la profession infirmière soit le niveau collégial et universitaire. Dans le premier cas, la formation mène à l'obtention d'un diplôme d'études collégiales et est d'une durée de 3 ans. Ce diplôme permet d'exercer au titre d'infirmière ou d'infirmier en soins généraux. Le second niveau de formation mène à l'obtention d'un baccalauréat et dure généralement trois ans. Un programme harmonisé permet aussi aux titulaires d'un diplôme d'études collégiales en soins infirmiers d'obtenir le baccalauréat en seulement deux ans. Le baccalauréat en sciences infirmières permet d'exercer à titre d'infirmière clinicienne ou d'infirmier clinicien, ou de conseillère ou conseiller en soins infirmiers.

\subsubsection{Les caractéristiques influençant l'étendue de la pratique infirmière}

L'état des connaissances a mis en évidence des caractéristiques susceptibles d'influencer l'étendue de la pratique infirmière des services de proximité en région éloignée. Le choix des caractéristiques a été guidé par le modèle ASCOP (Déry, 2013) qui a été développé en lien avec le QÉPI ainsi que par les travaux d'Aroke (2014) et tient compte de celles pouvant être facilement évaluables du point de vue des infirmières à l'aide d'un questionnaire auto administré. Ces caractéristiques susceptibles d'influencer le déploiement de la pratique infirmière sont regroupées, dans cette étude, en fonction du classement de Besner et al. (2005): soient les caractéristiques individuelles, professionnelles, organisationnelles et contextuelles en respect de la catégorisation issue de la revue de littérature présentée au tableau 2 dans le chapitre précédent. Les caractéristiques individuelles réfèrent ici aux caractéristiques sociodémographiques comme le sexe, l'âge et le niveau de formation. Les caractéristiques professionnelles font quant à elles référence au contexte professionnel lié à la profession infirmière, par exemple le fait de détenir ou non le droit de prescrire. Les caractéristiques organisationnelles sont 
celles en lien avec la vision, les ressources, la structure organisationnelle et les pratiques de l'organisation, comme le niveau de collaboration interprofessionnelle et finalement celles en lien avec le contexte sont associées aux particularités régionales.

\subsection{SCHEMATISATION DES LIENS ENTRE LES CONCEPTS A L'ETUDE}

Cette section présente la schématisation des liens entre les concepts à l'étude.

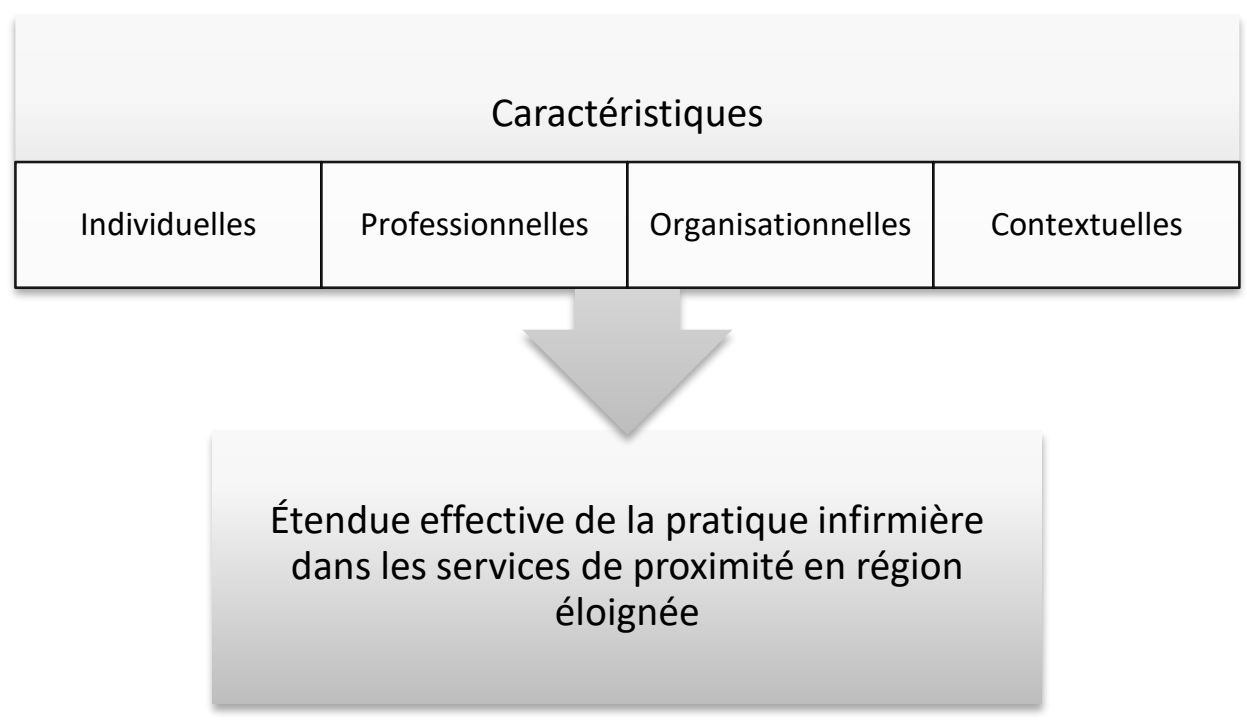

Figure 1. Cadre de référence de la présente étude 


\section{CHAPITRE 4}

\section{MÉTHODES}

Ce chapitre consacré aux aspects méthodologiques de la recherche présente le devis retenu et le milieu de la recherche, la population et l'échantillon, les méthodes de recrutement, la définition des variables à l'étude, les biais anticipés et stratégies pour les minimiser et le déroulement des activités. L'analyse des données, les considérations éthiques de même que les retombées attendues termineront ce chapitre.

\subsection{DEVIS DE RECHERCHE}

Un devis descriptif transversal a été retenu pour répondre aux objectifs de cette étude. Selon Groves, Burns et Gray (2013),

Le devis descriptif sert à explorer et à décrire des phénomènes dans des situations réelles. Cette approche est utilisée pour générer de nouvelles connaissances sur des concepts ou des sujets sur lesquels des recherches limitées ou inexistantes ont été menées. Grâce à une recherche descriptive, des concepts sont décrits et des relations sont identifiées qui servent de base à d'autres recherches quantitatives (Groves et al., 2013, page 215).

Dans cette étude, bien que les concepts soient bien connus, c'est le contexte de services de proximité en région éloignée qui l'est moins. Le devis descriptif a donc été sélectionné afin de décrire l'ÉEPI dans ce contexte encore peu étudié et d'identifier les caractéristiques l'influençant. L'aspect transversal implique, quant à lui, la présence d'un seul échantillon prélevé dans la population d'intérêt. 


\subsection{MILIEU DE RECHERCHE}

Le milieu de recherche sélectionné se situe dans les services de proximité d'un Centre intégré de santé et de services sociaux (CISSS) situé en région éloignée.

Le CISSS à l'étude, créé en 2015 suite à la fusion de six (6) centres de santé et de services sociaux, un centre de protection et de réadaptation et une agence de santé (MSSS, 2018) dessert la population habitant un vaste territoire s'étendant sur près de 236664 km2. Plusieurs municipalités de ce territoire se trouvent isolées n'étant pas reliées au réseau routier québécois. La région compte environ 83762 habitants répartis dans six municipalités régionales de comté (MRC). Les deux principaux centres urbains de la région qui ont respectivement des populations de 28417 et 31537 habitants (ministère des Affaires municipales et de l'habitation, 2018). Les Premières Nations représentant une population totale de 8290 habitants répartis à l'intérieur des 11 communautés autochtones sont également présentes dans cette région sanitaire (Ministère des Affaires municipales et de l'habitation, 2018).

Le CISSS à l'étude rencontre plusieurs défis causés par une pénurie de la maind'œuvre liés aux difficultés d'attraction et de rétention pour l'ensemble des professionnels de la santé ainsi qu'un taux d'inscription à la formation collégiale de soins infirmiers à la baisse (Paquet, 2018). Le CISSS connaît également des défis dans l'accès à un médecin où 2000 personnes sont actuellement sur la liste d'attente du guichet d'accès à un médecin de famille. Outre l'urgence, très peu de possibilités sont offertes à ces personnes pour accéder aux soins et services de santé. Dans cette région, de 2009 à 2014, le nombre total d'infirmières a chuté de $17 \%$ (Institut de la statistique du Québec, 2015). Différents modes de prestation de services de proximité sont présents, tels que des groupes de médecine familiale (4), des groupes de médecine familiale universitaire (1), des centres locaux de services communautaires (5) et des points de services CLSC éloignée communément appelés dispensaires (21), afin de répondre aux besoins de santé de la population. 


\subsection{Population et ÉChantillon}

Les infirmières pratiquant au sein des services de proximité dans le CISSS à l'étude constituent la population cible. Plus précisément, elles devaient répondre aux trois critères d'inclusion suivants:

- Détenir un permis de pratique valide de l'OIIQ;

- Occuper un poste ou un remplacement d'infirmière ou d'infirmière clinicienne et offrant des services à la clientèle;

- Travailler dans les services de proximité soit les CLSC (services de périnatalité, santé publique, soutien à domicile, soins de santé courants et santé mentale), les dispensaires ou dans les groupes de médecine familiale et groupes de médecine familiale universitaire (GMF et GMF-U); au sein du CISSS à l'étude.

Considérant ces critères, la population cible était composée de 133 infirmières au moment de débuter le recrutement (A. L., communication personnelle, 19 janvier 2018).

Un mode d'échantillonnage non probabiliste de type volontaire a été employé, c'est-à-dire que les participants ont été choisis selon leur disponibilité au moment de la collecte de données (Groves et al., 2013). Même si une étude similaire réalisée en Ontario a obtenu un taux de réponse très élevé soit 86,2 \% (Braithwaite, 2016), un taux de réponse conservateur de $30 \%$ paraissait réaliste dans le cadre de la présente étude étant donné que la tradition de recherche en sciences infirmières est encore en émergence dans la région. Un échantillon d'un minimum de 40 personnes était donc attendu.

\subsection{RECRUTEMENT}

À l'automne 2017, des contacts ont été établis avec les différents acteurs du milieu soit la directrice des soins infirmiers et des représentants des différentes directions concernées. Le recrutement a été réalisé via les canaux de communication du CISSS à l'étude sur une période d'un mois. Les critères d'inclusion ont été clairement présentés 
dans toutes les communications. Dans un premier temps, le 20 mars 2018, un courriel d'information a été envoyé aux gestionnaires afin de les informer de l'étude. Par la suite, le 21 mars, une communication de recrutement invitant les infirmières ciblées à participer à l'étude a été affichée sur l'intranet du CISSS à l'étude. Ce premier exercice a permis de recruter 15 répondants. Deux semaines plus tard, soit le 3 avril, les gestionnaires et les membres du comité exécutif du Conseil des infirmiers et infirmières de l'établissement ont été invités à partager via courriel la communication de rappel de recrutement aux infirmières ciblées. Dans la même semaine, la même communication est parue à l'intérieur de la publication électronique de l'établissement, soit l'info-CISSS. Ce deuxième exercice a permis d'augmenter le nombre de répondants à 34. Un dernier rappel a été envoyé aux gestionnaires dans les derniers jours, soit le 18 avril, les invitant à faire suivre la communication aux infirmières concernées. Ce dernier rappel a permis d'atteindre le minimum de répondants souhaités soit 40. La période de recrutement au sein du CISSS à l'étude a pris fin le 20 avril 2018 tel qu'il était prévu.

Afin d'augmenter le nombre de répondants, le recrutement s'est poursuivi par l'entremise du site Facebook de 1'Ordre régional des infirmières et infirmiers du Québec de la région à l'étude via lequel le document « communication courriel pour le recrutement des infirmières » a été utilisé et partagé le 29 avril 2018 pour finalement terminer la période de recrutement le 7 mai avec un nombre total de 49 répondants. Les communications utilisées pour le recrutement sont présentées à l'appendice VI.

Pour encourager la participation de la population cible, un tirage d'un chèque cadeau d'une valeur de 100 \$ à la fin de la période de recrutement parmi les répondants à l'étude a été réalisé. La transmission des coordonnées des participants pour le tirage du chèque cadeau a également permis de limiter le risque de double complétion sans l'éliminer toutefois complètement. À la toute fin de la période de recrutement et après la remise du chèque cadeau, soit le 7 mai 2018, un mot remerciant tous les participants de l'étude a été envoyé via tous les médias utilisés dans une communication générale sans qu'aucun nom de participant ne soit mentionné. 


\subsection{VARIABLES À L’ÉTUDE ET OUTILS DE COLLECTE DE DONNÉES}

Les variables à l'étude ont été regroupées en variable dépendante correspondant à l'ÉEPI et les variables indépendantes qui réfèrent aux caractéristiques individuelles, professionnelles, organisationnelles et contextuelles.

\subsubsection{Variable dépendante : L'étendue de la pratique des infirmières}

L'étendue effective de la pratique infirmière réfère à l'éventail des fonctions et responsabilités déployées par l'infirmière en lien avec ses compétences, son expérience et son champ d'exercices (D'Amour et al., 2012). Selon D'Amour et al. (2012), l'ÉEPI, qui est la variable dépendante à l'étude, se compose de six dimensions soit : 1- l'évaluation et la planification des soins; 2- l'enseignement à la clientèle et aux familles; 3- la communication et la coordination des soins; 4- l'intégration et l'encadrement du personnel; 5-1'optimisation de la qualité et de la sécurité des soins; et 6- la mise à jour et l'utilisation des connaissances.

Le QÉPI a été développé par D'Amour et al. (2012), à partir d'une analyse de la littérature pertinente, la consultation d'experts et des tests de validité dans le but de mesurer l'ÉEPI. Le QÉPI présente 26 questions de type Likert notées sur une échelle de 6 ( 1 , jamais; 2 , rarement; 3 , parfois; 4 , fréquemment; 5 , presque toujours, 6 , toujours) réparties d'une part, parmi les six dimensions composant l'étendue de la pratique infirmière et d'autre part pour trois niveaux de complexité des activités. Le niveau 1 (faible complexité) qui réfère au niveau de pratique de base attendu par les infirmières, y compris les novices. Le niveau 2 (complexité modérée) fait référence à des interventions plus complexes pouvant être effectuées par toutes les infirmières expérimentées, quelle que soit leur formation. Les éléments de niveau 3 (complexité élevée) sont des interventions nécessitant une formation universitaire ainsi qu'un bon niveau d'expérience. 
Les 26 questions associées à chaque dimension et niveau de complexité sont détaillées au tableau 3. Compte tenu que les scores de 5 et 6 correspondent respectivement à Presque toujours et Toujours, la logique veut qu'un score se rapprochant de 5 soit considéré comme un bon score alors qu'un score inférieur à 4, qui correspond à «Fréquemment » sera considéré comme un score où il y a place à amélioration.

Cet instrument a démontré une consistance interne acceptable avec un alpha de Cronbach global de 0,89 et variant de 0,61 à 0,70 pour les six dimensions (D'Amour et al., 2012). L'alpha de Cronbach permet d'apprécier la cohérence interne d'une échelle. Le QÉPI adapté au contexte de première ligne pour l'étude de Braithwaite a obtenu des scores allant de 0,66 à 0,91 .

À l'automne 2017, l'auteure principale du QÉPI (la professeure Danielle D'Amour) a été contactée afin d'obtenir son autorisation d'utiliser le questionnaire dans le cadre de la présente étude, de le modifier et de l'adapter au contexte culturel de la région à l'étude. Une fois cette autorisation obtenue, l'outil a été légèrement modifié pour s'assurer de répondre au contexte des services de proximité québécois en région éloignée. Pour ce, des modifications mineures ont été apportées à la version originale du QÉPI de D’Amour et al. (2012) en considérant la version adaptée au contexte de services de première ligne ontariens de Braithwaite (2016). La nouvelle version adaptée du questionnaire a ensuite été prétestée par trois infirmières détenant une expérience clinique ou de gestion dans les services de proximité en région éloignée $(n=3)$ et ne faisant pas partie de l'échantillon. Aucune modification n'a été nécessaire suite à cette validation. (Voir appendice III : adaptation du QÉPI). Parallèlement, le protocole de recherche ainsi que le questionnaire en ligne ont été conçus en collaboration avec la directrice de recherche. 


\subsubsection{Variables indépendantes : Caractéristiques individuelles, professionnelles, organisationnelles et contextuelles}

Les variables indépendantes susceptibles d'influencer l'étendue de la pratique infirmière sont divisées en 4 groupes soit les caractéristiques individuelles, professionnelles, organisationnelles et contextuelles. Le choix des variables a été guidé par le modèle ASCOP (Déry, 2013) qui a été développé en lien avec le QÉPI ainsi que par les travaux d'Aroke (2014) et tient compte de celles pouvant être facilement évaluables du point de vue des infirmières à l'aide d'un questionnaire auto-administré. Les caractéristiques individuelles sont : 1- le sexe auquel ils s'identifient (femme, homme), 2le groupe d'âge (moins de 35 ans, 35 à 50 ans et 50 ans et plus), 3- le nombre d'années d'expérience en tant qu'infirmière (moins de 5 ans ou 5 ans et plus), 4- le nombre d'années d'expérience dans les services de proximité (moins de 5 ans ou 5 ans et plus) et 5- le niveau de formation (titulaire ou non d'un baccalauréat). La caractéristique professionnelle est: 1- le fait de détenir le droit de prescrire. Les caractéristiques organisationnelles sont : 1- le poste occupé (infirmière ou infirmière clinicienne), 2- le statut d'emploi (permanent ou temporaire), 3- le fait de travailler à temps plein ou à temps partiel, 4- la fréquence de contact avec un médecin (tous les jours ou pas tous les jours), 5- le milieu de pratique (CLSC, dispensaire, GMF ou autres). Et enfin les caractéristiques contextuelles sont : 1- la distance en temps pour se rendre à un service d'urgence (à moins ou plus de 30 minutes de transport par la route) et 2- le lieu de travail qui a été déterminé en procédant au regroupement des six MRC de la région en fonction de trois contextes apparentés : 1) les MRC de l'Ouest ( $\mathrm{n}=2$ ) associé au premier pôle urbain, la MRC de l'Est $(\mathrm{n}=1)$ liée au deuxième pôle urbain de la région et 3) les MRC éloignées ( $\mathrm{n}=3$ ). Les données sur les caractéristiques individuelles, professionnelles, organisationnelles et contextuelles ont été intégrées dans la section «données sociodémographiques » situées au début du questionnaire. 
Tableau 3 : Dimensions, questions et niveaux de complexité des items du QÉPI

\begin{tabular}{|c|c|c|}
\hline Dimension & Questions & Niveau \\
\hline \multirow{5}{*}{$\begin{array}{l}\text { Évaluation et } \\
\text { planification des soins }\end{array}$} & $\begin{array}{l}\text { 25. J'évalue la condition physique et mentale du client en considérant les dimensions } \\
\text { biopsychosociales }\end{array}$ & \multirow{2}{*}{1} \\
\hline & $\begin{array}{l}\text { 21. Je mets à jour systématiquement, par écrit, les informations sur la condition du client et sur } \\
\text { les soins dispensés }\end{array}$ & \\
\hline & 2. Pour planifier mes interventions, j'utilise des outils d'évaluation de problèmes de soins & \multirow{2}{*}{2} \\
\hline & 1. Je suscite l'implication du client et de sa famille dans la planification des soins & \\
\hline & 11. Je participe à la conception, à l'application et à la mise à jour des programmes de soins & 3 \\
\hline \multirow{4}{*}{$\begin{array}{l}\text { Enseignement à la } \\
\text { clientèle et aux } \\
\text { familles }\end{array}$} & $\begin{array}{l}\text { 10. J'évalue les besoins spécifiques d'information et d'enseignement propres à chaque client et } \\
\text { à sa famille }\end{array}$ & \multirow[t]{2}{*}{1} \\
\hline & 17. Je valide la compréhension du client et de sa famille par rapport à l'enseignement reçu & \\
\hline & $\begin{array}{l}\text { 5. J'emploie des stratégies d'enseignement adaptées à chaque client et à sa famille, en fonction } \\
\text { du degré d'autonomie du client }\end{array}$ & 2 \\
\hline & 22. Je vérifie la qualité de l'enseignement dispensé à l'unité & 3 \\
\hline \multirow{5}{*}{$\begin{array}{l}\text { Communication et } \\
\text { coordination des } \\
\text { soins }\end{array}$} & $\begin{array}{l}\text { 16. Je communique aux membres de l'équipe toute information pertinente susceptible } \\
\text { d'influencer la coordination des soins }\end{array}$ & 1 \\
\hline & $\begin{array}{l}\text { 12. Je coordonne le travail de l'équipe de soins infirmiers pour répondre aux besoins du client } \\
\text { et de sa famille }\end{array}$ & \multirow{2}{*}{2} \\
\hline & $\begin{array}{l}\text { 24. Je communique toutes les informations pertinentes aux professionnels d'autres } \\
\text { établissements en vue d'assurer la continuité des soins }\end{array}$ & \\
\hline & 8. Je participe aux réunions ou à des activités de l'équipe interprofessionnelle & \multirow[b]{2}{*}{3} \\
\hline & $\begin{array}{l}\text { 15. Afin d'assurer la continuité des soins, je coordonne les interventions de l'équipe } \\
\text { interprofessionnelle au sein de l'établissement }\end{array}$ & \\
\hline \multirow{4}{*}{$\begin{array}{l}\text { Intégration et } \\
\text { encadrement du } \\
\text { personnel }\end{array}$} & 20. Je participe à l'identification des besoins de formation de mon unité & \multirow[b]{2}{*}{2} \\
\hline & $\begin{array}{l}\text { 9. Je participe à l'orientation et à l'encadrement des stagiaires ou du personnel nouvellement } \\
\text { recruté }\end{array}$ & \\
\hline & 6. J'agis à titre de mentor ou de monitrice auprès du personnel nouvellement recruté & \\
\hline & $\begin{array}{l}\text { 14. Je participe au développement et à la dispensation d'activités de formation à l'équipe de } \\
\text { soins, selon mes compétences }\end{array}$ & 3 \\
\hline \multirow{5}{*}{$\begin{array}{l}\text { Optimisation de la } \\
\text { qualité et sécurité des } \\
\text { soins }\end{array}$} & $\begin{array}{l}\text { 7. Je signale les situations cliniques où je perçois une lacune dans la qualité et la sécurité des } \\
\text { soins }\end{array}$ & 1 \\
\hline & $\begin{array}{l}\text { 26. Je m'implique dans la mise à jour des pratiques en vue d'améliorer la qualité et la sécurité } \\
\text { des soins }\end{array}$ & \multirow[t]{2}{*}{2} \\
\hline & $\begin{array}{l}\text { 19. Je propose des approches ou stratégies pour améliorer la qualité et la sécurité des soins } \\
\text { lorsque j’identifie des lacunes }\end{array}$ & \\
\hline & 23. Je participe à l'évaluation de la qualité et de la sécurité des soins & \multirow{2}{*}{3} \\
\hline & 3. Je participe au développement de la pratique infirmière & \\
\hline \multirow{3}{*}{$\begin{array}{l}\text { Mise à jour et } \\
\text { utilisation des } \\
\text { connaissances }\end{array}$} & 4. Je maintiens à jour mes connaissances & 1 \\
\hline & $\begin{array}{l}\text { 18. Je bonifie ma pratique en fonction des nouvelles connaissances issues des pratiques } \\
\text { exemplaires et des projets de recherche en sciences infirmières ou en santé }\end{array}$ & 2 \\
\hline & $\begin{array}{l}\text { 13. Lors d'une modification de la pratique, je partage avec l'équipe de soins infirmiers les } \\
\text { connaissances issues de la recherche }\end{array}$ & 3 \\
\hline
\end{tabular}




\subsubsection{Biais anticipés et stratégies pour les minimiser}

Pour la présente étude, certains biais ont été anticipés pour lesquels des stratégies ont été mises de l'avant dans le but de les minimiser.

En premier lieu, puisque l'échantillon de la présente étude en est un de convenance, un biais de sélection pouvait être présent et faire en sorte que celui-ci ne soit pas représentatif et homogène. Afin de minimiser les risques d'un biais méthodologique ayant pour effet un recrutement insuffisant, un chèque cadeau a été offert parmi les participants à l'étude. Cette stratégie a aussi permis de réduire le risque de biais de double complétion étant présent et a été diminuée en demandant aux participants leurs coordonnées pour le tirage du certificat cadeau. Cette stratégie a aussi permis de limiter l'effet d'un biais de désirabilité qui peut avoir été présent ainsi que le fait d'avoir un échantillon de volontaires possiblement davantage proactifs et ouverts à la recherche. Ce biais présuppose un comportement du participant consistant à se montrer sous une facette positive lorsqu'il est interrogé (Chabal, 2014). Les conséquences sur les résultats peuvent être un score plus élevé pour certaines dimensions ainsi que pour le score global de l'ÉEPI. Il faut aussi tenir compte que les résultats de cette étude découlent d'une pratique déclarée par les infirmières. Les résultats peuvent ainsi être teintés d'une part de subjectivité. Ce biais peut cependant être perçu comme une qualité de cette étude en ce sens que les infirmières ont eu l'opportunité de rapporter la situation telle qu'elles la perçoivent réellement. Cette possibilité a été prise en considération par une interprétation prudente des résultats.

\subsection{ANALYSE DES DONNÉES}

En premier lieu, des analyses descriptives ont été effectuées afin de décrire les caractéristiques de l'échantillon à l'aide de mesure de fréquence et pourcentage. Des mesures de tendances centrales ont été utilisées pour établir les scores du QÉPI (par 
activités, par dimensions, par niveau de complexité et global) suivi d'analyses comparatives. Puisque l'ensemble des variables des caractéristiques de l'échantillon répondaient aux postulats de bases soit le respect de la distribution normale des échantillons et l'homogénéité des variances, il a été possible d'utiliser des tests paramétriques (test-t et ANOVA) pour mener les analyses comparatives. Le logiciel SPSS 25 a été employé pour le traitement des données, leur classement et leur analyse.

\subsection{CONSIDÉRATIONS ÉTHIQUES}

Le protocole de recherche et les documents associés ont été déposés (questionnaire et communications) au comité central d'éthique de la recherche du ministère de la Santé et des Services sociaux au début février 2018. Le certificat éthique reçu au début mars 2018 (voir appendice IV : Certificat éthique) a ensuite été remis à la directrice des services multidisciplinaires, qualité, évaluation, performance et éthique du CISSS participant afin de procéder à un examen de la convenance institutionnelle (voir l'appendice V : Autorisation du milieu de recherche). À la mi-mars 2018, toutes les autorisations requises ayant été reçues, le recrutement a pu débuter.

Dans la préparation de ce protocole de recherche, un grand soin a été mis afin de respecter les principes éthiques associés au respect de la personne, à la préoccupation pour le bien-être et à la justice de 1'EPTC2 (http://eptc2fer.ca/welcome). Ainsi, le formulaire de consentement a été présenté aux participants au début du questionnaire électronique afin de s'assurer de leur volontariat (voir l'appendice VII : Formulaire de consentement). Ceux-ci ont été avisés de la possibilité de se retirer à tout moment de l'étude, et ce, sans aucun préjudice. Les noms et les coordonnées de l'étudiante-chercheure, de la directrice de recherche, de la directrice des services multidisciplinaires, qualité, évaluation, performance et éthique du CISSS participant et d'une personne-ressource au comité central d'éthique de la recherche du MSSS leur ont également été fournies et ils ont été 
invités à les contacter en cas de questionnements sur le projet. Par la suite, la nature de la participation au projet a été décrite, soit la complétion d'un questionnaire en ligne d'une durée approximative de vingt minutes. La confidentialité des données a été assurée puisqu'aucune donnée nominative ne leur a été demandée, sauf leur adresse courriel qui a servi uniquement à l'attribution du prix de 100 \$ tiré au sort. Afin qu'aucune corrélation ne soit possible entre les questionnaires et les adresses courriel recueillis, un hyperlien dirigeant les personnes intéressées à participer au tirage vers un site externe à la fin du sondage afin qu'elles puissent transmettre leurs coordonnées de façon volontaire si elles souhaitaient participer au concours. Le tirage du gagnant s'est effectué en présence de la directrice de recherche et de l'étudiante. Cette dernière a transmis le chèque cadeau d'une valeur de 100 \$ provenant de chez Archambault au gagnant. L'étude n’a présenté aucun risque et très peu d'inconvénients outre le temps consacré pour répondre au questionnaire et le fait que cela ait pu générer de la fatigue et/ou un certain malaise lié aux réponses. Les résultats générés par cette étude ne permettent en aucun temps de pouvoir identifier de façon personnelle un participant en particulier. Dans le même ordre d'idées, le CISSS à l'étude ainsi que les services sélectionnés ne sont pas explicitement nommés dans ce mémoire et dans les publications futures. Toutes les données seront conservées cinq ans après l'acceptation du mémoire sur une clé USB protégée par mot de passe. 


\section{CHAPITRE 5 \\ RÉSULTATS}

Ce chapitre présente les résultats de l'étude en trois sections distinctes permettant de répondre aux deux objectifs de la recherche présentés à la section 2.5 qui étaient de 1) mesurer l'étendue de la pratique infirmière dans les services de proximité de la région éloignée à l'étude et 2) identifier les caractéristiques individuelles, professionnelles, organisationnelles et contextuelles influençant cette étendue dans le contexte des services de proximité de la région éloignée à l'étude. La première section porte sur les caractéristiques de l'échantillon. La deuxième concerne le score global de l'étendue effective de la pratique ainsi que les scores pour chaque dimension et pour chaque niveau de complexité. La dernière section présente les résultats des analyses comparatives des caractéristiques individuelles, professionnelles, organisationnelles ou contextuelles susceptibles d'influencer l'ÉEPI.

\subsection{CARACTÉristiQues de L'ÉCHANTILlon}

Quarante-neuf questionnaires ont été remplis. Cinq ont été rejetés, car ils ne respectaient pas les critères d'inclusion pour les raisons suivantes: travaillant auprès d'un autre CISSS ( $\mathrm{n}=1)$ ou ne travaillant pas dans les services de proximité $(\mathrm{n}=3)$ ou n'offrant pas de soin direct à la clientèle $(n=1)$. L'échantillon final est donc constitué de 44 participants, ce qui correspond à un taux de réponse de $33 \%$.

Les caractéristiques des répondants figurent dans la première colonne des scores du tableau 5. La majorité des participants sont de sexe féminin (86,4\%), font partie du groupe d'âge des 35 à 50 ans (54,5\%), cumulent plus de 15 ans d'expérience comme infirmières $(54,5 \%)$ et plus de 5 ans dans les services de proximité $(59,1 \%)$. Ils sont 25 
$(56,8 \%)$ à détenir un baccalauréat en sciences infirmières tandis que le reste de l'échantillon détient une formation de niveau collégial.

Elles sont $63,6 \%$ à occuper un poste d'infirmière clinicienne, plus de $70 \%$ travaillent à temps complet et ont un statut d'emploi permanent. La majorité ne détient pas leur droit de prescrire, soit 56,8\%. L'échantillon est bien réparti entre les regroupements de MRC. Ainsi, le principal lieu de travail des répondantes se situe dans les MRC de l'Ouest (sous-région 1) pour 43,2 \%, dans la MRC de l'Est (sous-région 2) pour $25 \%$ et dans les MRC éloignées (sous-région 3) pour 31,8\%. Le milieu de pratique de la majorité des répondantes est le CLSC, soit 52,3\%, alors que 34,1\% d'entre elles pratiquent en dispensaire et 6,8\% en GMF ou GMF-U. Pour la grande majorité, leur milieu de pratique se situe à moins de 30 minutes de distance d'un service d'urgence, soit $68,2 \%$. Tous les répondants indiquent collaborer avec un médecin dans leur pratique. 22,7\% disent avoir un contact moins d'une fois par semaine avec le médecin, 43,2 \% disent avoir un contact, mais pas tous les jours et $34,1 \%$ chaque jour.

\subsection{EXAMEN DE L'ÉTENDUE EFFECTIVE DE PRATIQUE DES INFIRMIÈRES}

La corrélation inter-dimensions et les corrélations interactivités associées à chaque dimension ont été réalisées à l'aide de l'indice de l'alpha de Cronhbach $(\alpha)$ pour procéder à l'examen de la cohérence interne des scores obtenus avec le QÉPI-modifié. L'examen de la corrélation inter-dimension indique un indice $\alpha$ de 0,91 pour le score global, ce qui est similaire à celui de D'Amour et al. (2012). Pour l'examen des dimensions, les indices $\alpha$ varient entre 0,617 et 0,780 pour les dimensions et entre 0,769 et 0,823 pour les différents niveaux de complexité. Les moyennes, écarts-types et étendues (min-max) des scores pour chacune des 26 activités du questionnaire, pour chacune des six dimensions, pour chacune des trois niveaux de complexité et le score global sont détaillés au tableau 4. Le score global de l'ÉEPI est de 4,15 (écart-type $[\sigma]=0,85$ ), ce qui signifie que les infirmières travaillant au sein des services de proximité de la région à l'étude réalisent 
fréquemment les activités reliées à leur champ de pratique. Ce score est donc satisfaisant. Tel que mentionné précédemment, dans le cadre de cette étude, il a été considéré qu'un score supérieur à 5 est très satisfaisant, entre 4 et 5 satisfaisant et inférieur à 4 sousoptimal.

L'examen des scores par dimension a toutefois mis en évidence des différences entre les dimensions. Ainsi, les dimensions ayant obtenue les scores les plus élevés et supérieures à 4 équivalents à la cote «satisfaisant » sont l' «Évaluation et planification des soins » $(\mathrm{x}=4,62, \sigma=0,84), \mathrm{l}^{\prime}$ « Enseignement à la clientèle et aux familles » $(\mathrm{x}=4,59, \sigma$ $=0,98)$, la «Mise à jour et utilisation des connaissances $»(\mathrm{x}=4,58, \sigma=0,98)$ et la «Communication et coordination des soins $»(x=4,00, \sigma=1,18)$. Les dimensions de l'ÉEPI ayant obtenu des scores inférieurs à 4 laissant penser que des améliorations sont souhaitables sont «l" "Optimisation de la qualité et de la sécurité des soins » $(\mathrm{x}=3,82, \sigma$ $=1,14)$ et l' «Intégration et encadrement du personnel $»(\mathrm{x}=3,40, \sigma=1,30)$.

Sans surprise, les scores établis en fonction des niveaux de complexité décroissent à mesure que le niveau de complexité s'accroît. Autrement dit, plus l'activité est complexe, plus le score de l'ÉEPI est faible. Ainsi, pour le niveau de complexité faible, la moyenne des scores était de 4,99 $(\sigma=0,83)$, ce qui correspond à «presque toujours » dans l'échelle de Likert. Pour le niveau de complexité moyenne, le score moyen est de 4,22 ( $\sigma$ $=0,84)$ pour le niveau de complexité modéré et de 3,40 $(\sigma=1,09)$ pour le niveau d'activités plus complexes, soient celles requérant une formation universitaire. 
Tableau 4 : Score moyens de l’ÉEPI globale, de ses dimensions, questions et niveaux de complexité

\begin{tabular}{|c|c|c|c|c|c|}
\hline Variable & $\begin{array}{c}\text { Nombre de } \\
\text { variables }\end{array}$ & Min & Max & $\begin{array}{l}\text { Moyenne } \\
\text { (x) }\end{array}$ & $\begin{array}{c}\text { Écart-type } \\
(\sigma)\end{array}$ \\
\hline \multicolumn{6}{|l|}{ Score global } \\
\hline Étendue effective de la pratique & 26 & 2,00 & 5,73 & 4,15 & $\mathbf{0 , 8 5}$ \\
\hline \multicolumn{6}{|l|}{ Dimensions } \\
\hline 1- Évaluation et planification des soins & 5 & 2,60 & 6,00 & 4,62 & $\mathbf{0 , 8 4}$ \\
\hline Q1 - Planification soins & 1 & 1 & 6 & 4,70 & 1,34 \\
\hline Q2 - Utilisation outils d'évaluation & 1 & 2 & 6 & 4,68 & 1,1 \\
\hline Q11 - Conception et mises à jour programmes de soins & 1 & 1 & 6 & 3,14 & 1,79 \\
\hline Q21 - Documentation régulière de la condition du client & 1 & 3 & 6 & 5,32 & 0,91 \\
\hline Q25 - Évaluation autonome de la condition & 1 & 3 & 6 & 5,25 & 1,04 \\
\hline 2- Enseignement à la clientèle et aux familles & 4 & 2,00 & 6,00 & 4,59 & ,98 \\
\hline Q5 - Stratégies enseignement adaptées & 1 & 3 & 6 & 5,16 & 0,94 \\
\hline Q10 - Évaluer besoins d'information & 1 & 2 & 6 & 4,57 & 1,17 \\
\hline Q17 - Adaptation des interventions éducatives & 1 & 2 & 6 & 5,05 & 1,20 \\
\hline Q22 - Évaluation et amélioration enseignement & 1 & 1 & 6 & 3,57 & 1,70 \\
\hline 3- Communication et coordination des soins & 5 & 1,60 & 5,80 & 4,00 & 1,18 \\
\hline Q8 - Participe réunions équipe & 1 & 1 & 6 & 4,16 & 1,87 \\
\hline Q12 - Coordination équipe soins & 1 & 1 & 6 & 3,14 & 1,75 \\
\hline Q15 - Coordination équipe interprofessionnelle & 1 & 1 & 6 & 3,27 & 1,91 \\
\hline Q16 - Communication équipe & 1 & 1 & 6 & 4,77 & 1,6 \\
\hline Q24 - Continuité soins inter établissements & 1 & 1 & 6 & 4,66 & 1,54 \\
\hline 4- Intégration et encadrement du personnel & 4 & 1,25 & 5,75 & $\mathbf{3 , 4 0}$ & 1,30 \\
\hline Q6 - Mentorat ou monitorat & 1 & 1 & 6 & 3,43 & 1,61 \\
\hline Q9 - Orientation et formation de la relève infirmière & 1 & 1 & 6 & 3,30 & 1,84 \\
\hline Q14 - Conception, développement dispensation activités de formation & 1 & 1 & 6 & 3,16 & 1,74 \\
\hline Q20 - Identification besoin formation & 1 & 1 & 6 & 3,70 & 1,50 \\
\hline 5- Optimisation de la qualité et de la sécurité des soins & 5 & 1,40 & 6,00 & 3,82 & 1,14 \\
\hline Q3 - Développement et amélioration pratique infirmière & 1 & 1 & 6 & 2,89 & 1,67 \\
\hline Q7 - Signalement des lacunes cliniques & 1 & 2 & 6 & 4,91 & 1,38 \\
\hline Q19 - Amélioration qualité et sécurité soins & 1 & 1 & 6 & 4,52 & 1,37 \\
\hline Q23 - Évaluation qualité sécurité soins & 1 & 1 & 6 & 2,80 & 1,66 \\
\hline Q26 - Mise à jour pratiques & 1 & 1 & 6 & 3,98 & 1,82 \\
\hline 6- Mise à jour et utilisation des connaissances & 3 & 1,67 & 6,00 & 4,58 & ,98 \\
\hline Q4 - Connaissances actualisées & 1 & 3 & 6 & 5,09 & 0,94 \\
\hline Q13 - Promotion des connaissances issues de la recherche & 1 & 1 & 6 & 4,25 & 1,63 \\
\hline Q18 - Bonification de la pratique & 1 & 1 & 6 & 4,39 & 1,24 \\
\hline \multicolumn{6}{|l|}{ Niveau de complexité } \\
\hline 1- Niveau faible (Q25, Q21, Q10, Q17, Q16, Q7, Q4) & 7 & 2,71 & 6,00 & 4,99 &, 83 \\
\hline 2- Niveau moyen (Q2, Q1, Q5, Q12, Q24, Q20, Q9, Q26, Q19, Q18) & 10 & 2,20 & 6,00 & 4,22 &, 84 \\
\hline 3- Niveau élevé (Q11, Q22, Q8, Q15, Q6, Q14, Q23, Q3, Q13) & 9 & 1,22 & 5,56 & 3,41 & 1,09 \\
\hline
\end{tabular}




\subsection{L'ÉTENDUE EFFECTIVE DE LA PRATIQUE INFIRMIÈRES EN FONCTION DES CARACTÉRISTIQUES INDIVIDUELLES, PROFESSIONNELLES, ORGANISATIONNELLES ET CONTEXTUELLES}

Les analyses comparatives des caractéristiques individuelles, professionnelles, organisationnelles et contextuelles, indiquent que le résultat global ainsi que des dimensions de l'ÉEPI sont influencés par certaines caractéristiques étudiées. (Voir tableaux 5 et 6 ). Ainsi, le score des femmes est significativement plus élevé ( $x=4,15, \sigma$ $=1,14$ ) pour les activités liées à la dimension « communication et coordination des soins » que celui des hommes $(\mathrm{x}=3,07, \sigma=1,07)$. Travailler à temps plein est associé à des scores supérieurs pour les dimensions «intégration et encadrement du personnel» et « optimisation de la qualité et de la sécurité des soins » $(\mathrm{x}=3,79, \sigma=1,21$ et $\mathrm{x}=4,04$, $\sigma=1,02)$ que travailler à temps partiel $(\mathrm{x}=2,46, \sigma=1,04$ et $\mathrm{x}=3,29, \sigma=1,27)$. Les analyses comparatives démontrent aussi une différence significative entre les lieux de travail pour la dimension « communication et coordination des soins ». La considération de test post-hoc de Bonferroni $(\mathrm{p}=0,012)$ indique que la sous-région 3, soit les MRC les plus éloignées et isolées obtient des scores significativement plus faibles $(x=3,26, \sigma=$ 1,21) que la sous-région $1(\mathrm{x}=4,42, \sigma=0,91)$ pour cette dimension. Également, les analyses démontrent une différence significative pour le score global entre les infirmières ne détenant pas le droit de prescrire $(\mathrm{x}=3,87, \sigma=0,81)$ et celles détenant ce droit $(\mathrm{x}=4,51, \sigma=0,77)$ avec un plus grand déploiement de la pratique pour ces dernières.

En ce qui concerne les niveaux de complexité, les résultats démontrent que le lieu de travail semble associé à une étendue de la pratique plus élevée pour les activités de haut niveau de complexité dans la sous-région $2(\mathrm{x}=4,04, \sigma=1,22)$ qui obtient des scores significativement plus élevés (Bonferroni $\mathrm{p}=0,027$ ) que la sous-région 3 ( $\mathrm{x}=2,91$, $\sigma=1,06$ ) représentant les MRC plus éloignées et isolées (tableau 7). 
Tableau 5 : Comparaison du score global en fonction des caractéristiques

\begin{tabular}{|c|c|c|c|c|c|}
\hline & $\mathbf{N}(\%)$ & $x$ & E.T. & ddl & $t / F$ \\
\hline \multicolumn{6}{|l|}{ Sexe } \\
\hline Féminin & $38(86,4)$ & 4,22 & 0,85 & 42 & 1,52 \\
\hline Masculin & $6(13,6)$ & 3,67 & 0,69 & & \\
\hline \multicolumn{6}{|l|}{ Âge } \\
\hline - de 35 ans & $9(20,5)$ & 4,16 & 0,96 & & \\
\hline De 35 à 50 ans & $24(54,5)$ & 4,08 & 0,82 & 2 & 0,77 \\
\hline 51 ans et + & $11(25)$ & 4,05 & 0,85 & & \\
\hline \multicolumn{6}{|l|}{ Expérience totale } \\
\hline - de 5 ans & $6(13,6)$ & 4,37 & 0,94 & 42 & 0.69 \\
\hline 5 ans et + & $38(86,4)$ & 4,11 & 0,84 & & \\
\hline \multicolumn{6}{|l|}{ Expérience en SP } \\
\hline - de 5 ans & $18(40,9)$ & 4,28 & 0,77 & 42 & 0,89 \\
\hline 5 ans et + & $26(59,1)$ & 4,05 & 0,90 & & \\
\hline \multicolumn{6}{|l|}{ Formation } \\
\hline Pas bac & $19(43,2)$ & 4,19 & 0,91 & 42 & 0,27 \\
\hline Baccalauréat & $25(56,8)$ & 4,12 & 0,81 & & \\
\hline \multicolumn{6}{|l|}{ Poste } \\
\hline Infirmière & $16(36,4)$ & 4,36 & 0,88 & 42 & 1,25 \\
\hline Inf. clinicienne & $28(63,6)$ & 4,03 & 0,82 & & \\
\hline \multicolumn{6}{|l|}{ Statut d'emploi } \\
\hline Temporaire & $13(29,5)$ & 4,01 & 0,96 & 42 & 0,68 \\
\hline Permanent & $31(70,5)$ & 4,20 & 0,80 & & \\
\hline \multicolumn{6}{|l|}{ Travail } \\
\hline Temps plein & $31(70,5)$ & 4,30 & 0,78 & 42 & 1,83 \\
\hline Temps partiel & $13(29,5)$ & 3,80 & 0,94 & & \\
\hline \multicolumn{6}{|l|}{ Droit de prescrire } \\
\hline Oui & $19(43,2)$ & 4,51 & 0,77 & 42 & $2,62 *$ \\
\hline Non & $25(56,8)$ & 3,87 & 0,81 & & \\
\hline \multicolumn{6}{|l|}{ Lieu de travail } \\
\hline Sous-région 1 & $19(43,2)$ & 4,17 & 0,67 & ? & \\
\hline Sous-région 2 & $11(25,0)$ & 4,58 & 0,91 & 2 & 3,01 \\
\hline Sous-région 3 & $14(31,8)$ & 3,78 & 0,90 & & \\
\hline \multicolumn{6}{|l|}{ Milieu de pratique } \\
\hline CLSC & $23(52,3)$ & 4,21 & 0,84 & & \\
\hline$G M F, G M F-U$ & $3(6,8)$ & 4,19 & 0,55 & 3 & 1,54 \\
\hline Dispensaires & $15(34,1)$ & 3,88 & 0,88 & & \\
\hline Autres & $3(6,8)$ & 4,97 & 0,65 & & \\
\hline \multicolumn{6}{|l|}{ Distance de l'urgence } \\
\hline 30 min. et - & $30(68,2)$ & 4,27 & 0,80 & 42 & 1,42 \\
\hline + de 30 min. ou non relié & $14(31,8)$ & 3,88 & 0,91 & & \\
\hline \multicolumn{6}{|l|}{ Collaboration avec médecin } \\
\hline Quotidien & $15(34,1)$ & 4,34 & 0,80 & 42 & 1,08 \\
\hline Non quotidien & $2965,9)$ & 4,05 & 0,87 & & \\
\hline
\end{tabular}

Note. Le degré de liberté (ddl) désigne le nombre de variables aléatoires qui ne peuvent être déterminées ou fixées par une équation. Celui-ci est égal au nombre d'observations moins le nombre de relations entre ces observations. Les scores établis en fonction $\mathrm{ddl}=42$ ont été établis en fonction de $\mathrm{t}$-test et tandis que pour les autres ddl, les scores ont été établis à l'aide d'ANOVAs. Le tVF désigne la valeur t pour les t-test et la valeur F pour les Anovas. Ceux-ci sont exprimés par rapport à la première variable nommée.

$* \mathrm{p}<0,05 \quad * * \mathrm{p}<0,01$ 
Tableau 6 : Comparaison des différentes dimensions en fonction des caractéristiques

\begin{tabular}{|c|c|c|c|c|c|c|c|c|c|c|c|c|c|c|c|c|c|c|c|c|}
\hline \multirow{3}{*}{ Caractéristiques } & \multirow[b]{3}{*}{$\mathrm{N}(\%)$} & \multirow[b]{3}{*}{ ddl } & \multicolumn{18}{|c|}{ Dimensions } \\
\hline & & & \multicolumn{3}{|c|}{1} & \multicolumn{3}{|c|}{2} & \multicolumn{3}{|c|}{3} & \multicolumn{3}{|c|}{4} & \multicolumn{3}{|c|}{5} & \multicolumn{3}{|c|}{6} \\
\hline & & & $\mathrm{x}$ & $\sigma$ & $t \backslash F$ & $\mathrm{x}$ & $\sigma$ & $t \backslash F$ & $\mathrm{x}$ & $\sigma$ & $t \backslash F$ & $\mathrm{x}$ & $\sigma$ & $t \backslash F$ & $\mathrm{x}$ & $\sigma$ & $\mathrm{t} \backslash \mathrm{F}$ & $\mathrm{x}$ & $\sigma$ & $t \backslash F$ \\
\hline Sexe & & & & & & & & & & & & & & & & & & & & \\
\hline Féminin & $38(86,4)$ & 42 & 4,65 & 0,86 & 0,58 & 4,59 & 1,00 & 0,005 & 4,15 & 1,14 & $2,18^{*}$ & 3,47 & 1,34 & 0,89 & 3,94 & 1,12 & 1,87 & 4,64 & 1,00 & 1,11 \\
\hline Masculin & $6(13,6)$ & & 4,43 & 0,77 & & 4,58 & 0,97 & & 3,07 & 1,07 & & 2,96 & 1,03 & & 3,03 & 0,99 & & 4,17 & 0,84 & \\
\hline \multicolumn{21}{|l|}{ Âge } \\
\hline - de 35 ans & $9(20,5)$ & \multirow{3}{*}{2} & 471 & 0,88 & \multirow{3}{*}{0,63} & 4,86 & 0,90 & \multirow{3}{*}{1,23} & 4,44 & 0,80 & \multirow{3}{*}{1,18} & 3,72 & 1,53 & \multirow{3}{*}{0,42} & 4,13 & 1,37 & \multirow{3}{*}{1,51} & 5,07 & 0,88 & \multirow{3}{*}{2,62} \\
\hline De 35 à 50 ans & $24(54,5)$ & & 449 & 0,85 & & 4,38 & 0,97 & & 4,00 & 1,29 & & 3,38 & 1,33 & & 3,93 & 1,05 & & 4,29 & 0,93 & \\
\hline 51 ans et + & $11(25)$ & & 4,82 & 0,82 & & 4,82 & 1,04 & & 3,64 & 1,13 & & 3,18 & 1,08 & & 3,33 & 1,09 & & 4,79 & 1,02 & \\
\hline \multicolumn{21}{|l|}{ Expérience totale } \\
\hline - de 5 ans & $6(13,6)$ & \multirow[t]{2}{*}{42} & 4,87 & 0,74 & \multirow[t]{2}{*}{0,78} & 4,83 & 0,98 & \multirow[t]{2}{*}{0,66} & 4,50 & 0,75 & 1,12 & 3,38 & 1,59 & $-0,05$ & 3,93 & 1,40 & 0,26 & 4,78 & 1,00 & 0,54 \\
\hline 5 ans et + & $38(86,4)$ & & 4,58 & 0,86 & & 4,55 & 1,00 & & 3,92 & 1,22 & & 3,40 & 1,28 & & 3,80 & 1,11 & & 4,54 & 0,98 & \\
\hline Expérience en SP & & & & & & & & & & & & & & & & & & & & \\
\hline- de 5 ans & $18(40,9)$ & & 4,57 & 0,75 & & & 0,80 & & 4,41 & & & 3,65 & 1,42 & & 3,89 & & & 4,57 & 1,05 & \\
\hline 5 ans et + & $26(59,1)$ & 42 & 4,65 & 0,91 & $-0,34$ & 4,52 & 1,10 & 0,53 & 3,72 & 0,93 & 1,99 & 3,22 & 1,21 & 1,08 & 3,77 & 1,16 & 0,34 & 4,58 & 0,95 & $-0,009$ \\
\hline & & & & & & & & & & 1,26 & & & & & & & & & & \\
\hline Formation & & & & & & & & & & & & & & & & & & & & \\
\hline Pas bac & $19(43,2)$ & 42 & 4,76 & 0,93 & 0,96 & 4,50 & 1,09 & $-0,50$ & 4,09 & 1,28 & 0,46 & 3,38 & 1,47 & $-0,07$ & 3,82 & 1,17 & 0,01 & 4,67 & 1,02 & 0,53 \\
\hline Baccalauréat & $25(56,8)$ & & 4,51 & 0,77 & & 4,65 & 0,91 & & 3,93 & 1,12 & & 3,41 & 1,19 & & 3,82 & 1,14 & & 4,51 & 0,96 & \\
\hline Poste & & & & & & & & & & & & & & & & & & & & \\
\hline Infirmière & $16(36,4)$ & 42 & 4,88 & 0,97 & 1,56 & 4,59 & 1,13 & 0,04 & 4,25 & 1,23 & 1,07 & 3,64 & 1,45 & 0,94 & 4,01 & 1,08 & 0,85 & 4,90 & 0,94 & 1,67 \\
\hline Inf. clinicienne & $28(63,6)$ & & 4,47 & 0,74 & & 4,58 & 0,91 & & 3,86 & 1,14 & & 3,26 & 1,21 & & 3,71 & 1,18 & & 4,39 & 0,97 & \\
\hline Statut d'emploi & & & & & & & & & & & & & & & & & & & & \\
\hline Temporaire & $13(29,5)$ & 42 & 4,49 & 0,85 & $-0,64$ & 4,13 & 1,09 & $-2,04$ & 3,98 & 1,33 & $-0,06$ & 3,31 & 1,52 & $-0,29$ & 3,83 & 1,19 & 0,05 & 4,33 & 0,97 & $-1,07$ \\
\hline Permanent & $31(70,5)$ & & 4,67 & 0,84 & & 4,77 & 0,89 & & 4,01 & 1,13 & & 3,44 & 1,22 & & 3,81 & 1,14 & & 4,68 & 0,98 & \\
\hline Travail & & & & & & & & & & & & & & & & & & & & \\
\hline Temps plein & $31(70,5)$ & 42 & 4,70 & 0,75 & 0,96 & 4,71 & 0,96 & 1,31 & 4,05 & 1,19 & 0,39 & 3,79 & 1,21 & $3,46 * *$ & 4,04 & 1,02 & $2,06 *$ & 4,59 & 0,96 & 0,16 \\
\hline Temps partiel & $13(29,5)$ & & 4,43 & 1,04 & & 4,29 & 1,01 & & 3,89 & 1,19 & & 2,46 & 1,04 & & 3,29 & 1,27 & & 4,54 & 1,05 & \\
\hline Droit de prescrire & & & & & & & & & & & & & & & & & & & & \\
\hline Oui & $19(43,2)$ & 42 & 4,89 & 0,80 & 1,97 & 4,96 & 0,91 & $2,32^{*}$ & 4,27 & 1,11 & 1,36 & 3,79 & 1,08 & 1,79 & 4,33 & 1,01 & $2,77^{* *}$ & 4,91 & 0,79 & 2,06 \\
\hline Non & $25(56,8)$ & & 4,41 & 0,82 & & 4,30 & 0,95 & & 3,79 & 1,21 & & 3,10 & 1,39 & & 3,43 & 1,10 & & 4,32 & 1,04 & \\
\hline
\end{tabular}




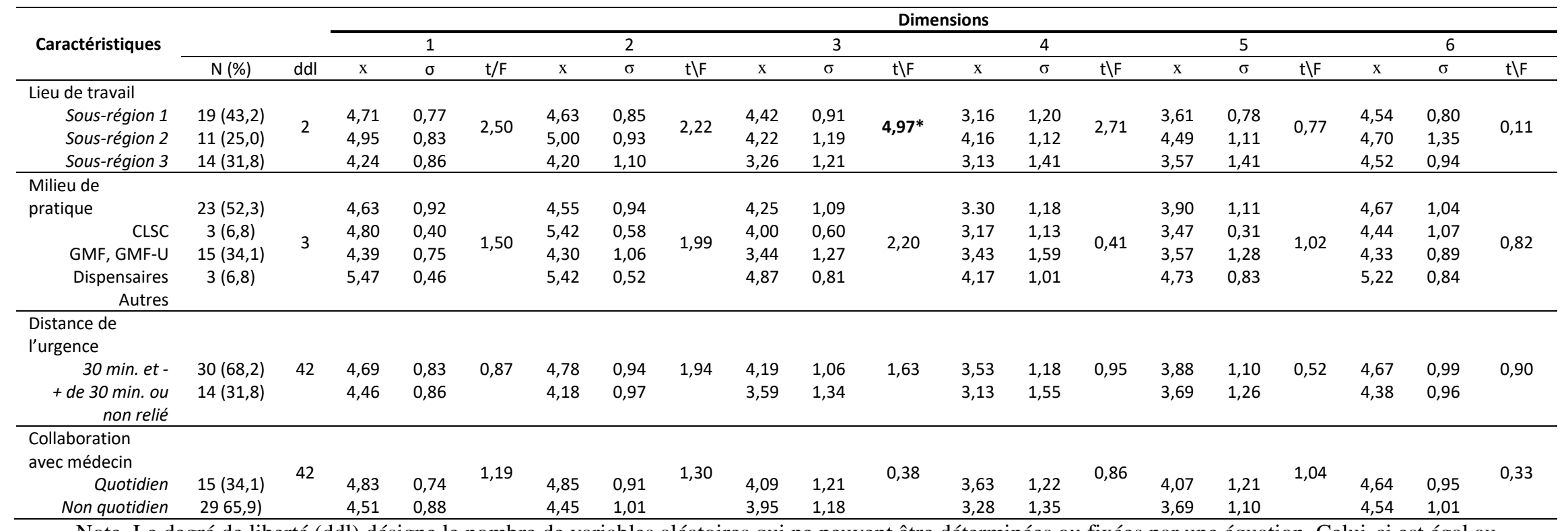

Note. Le degré de liberté (ddl) désigne le nombre de variables aléatoires qui ne peuvent être déterminées ou fixées par une équation. Celui-ci est égal au nombre d'observations moins le nombre de relations entre ces observations. Les scores établis en fonction ddl = 42 ont été établis en fonction de t-test et tandis que pour les autres ddl, les scores ont été établis à l'aide d'ANOVAs. Le tlF désigne la valeur t pour les t-test et la valeur F pour les Anovas. Ceuxci sont exprimés par rapport à la première variable nommée.

$* \mathrm{p}<0,05 \quad * * \mathrm{p}<0,01$ 
Tableau 7 : Comparaison des niveaux de complexité avec les caractéristiques à l'étude

\begin{tabular}{|c|c|c|c|c|c|c|c|c|c|c|c|}
\hline \multirow{3}{*}{ Caractéristiques } & \multirow[b]{3}{*}{$N(\%)$} & \multirow[b]{3}{*}{ ddl } & \multicolumn{9}{|c|}{ Niveaux de complexité } \\
\hline & & & \multicolumn{3}{|c|}{1} & \multicolumn{3}{|c|}{2} & \multicolumn{3}{|c|}{3} \\
\hline & & & $\mathrm{x}$ & $\sigma$ & $t / F$ & $\mathrm{x}$ & $\sigma$ & $t / F$ & $\mathrm{x}$ & $\sigma$ & $t / F$ \\
\hline \multicolumn{12}{|l|}{ Sexe } \\
\hline Féminin & $38(86,4)$ & 42 & 5,05 & 0,84 & 1,19 & 4,29 & 0,85 & 1,29 & 3,51 & 1,10 & 1,60 \\
\hline Masculin & $6(13,6)$ & & 4,62 & 0,72 & & 3,82 & 0,67 & & 2,76 & 0,79 & \\
\hline \multicolumn{12}{|l|}{ Âge } \\
\hline - de 35 ans & $9(20,5)$ & & 5,30 & 0,76 & & 4,47 & 0,90 & & 3,80 & 1,33 & \\
\hline De 35 à 50 ans & $24(54,5)$ & 2 & 4,87 & 0,84 & 0,88 & 4,17 & 0,87 & 0,47 & 3,36 & 1,01 & 0,83 \\
\hline 51 ans et + & $11(25)$ & & 5,01 & 0,87 & & 4,15 & 0,77 & & 3,19 & 1,05 & \\
\hline \multicolumn{12}{|l|}{ Expérience totale } \\
\hline- de 5 ans & $6(13,6)$ & 42 & 5,38 & 0,58 & 1,23 & 4,33 & 0,96 & 0,34 & 3,63 & 1,37 & 0,54 \\
\hline 5 ans et + & $38(86,4)$ & & 4,93 & 0,86 & & 4,21 & 0,83 & & 3,37 & 1,05 & \\
\hline \multicolumn{12}{|l|}{ Expérience en SP } \\
\hline - de 5 ans & $18(40,9)$ & 42 & 5,20 & 0,59 & 1,50 & 4,30 & 0,78 & 0,50 & 3,56 & 1,13 & 0,75 \\
\hline 5 ans et + & $26(59,1)$ & & 4,85 & 0,95 & & 4,17 & 0,89 & & 3,30 & 1,07 & \\
\hline \multicolumn{12}{|l|}{ Formation } \\
\hline Pas Bac & $19(43,2)$ & 42 & 5,05 & 0,87 & 0,41 & 4,23 & 0,88 & 0,02 & 3,47 & 1,16 & 0,35 \\
\hline Baccalauréat & $25(56,8)$ & & 4,95 & 0,82 & & 4,22 & 0,82 & & 3,36 & 1,05 & \\
\hline \multicolumn{12}{|l|}{ Poste } \\
\hline Infirmière & $16(36,4)$ & 42 & 5,21 & 0,84 & 1,34 & 4,35 & 0,91 & 0,76 & 3,70 & 1,08 & 1,38 \\
\hline Inf. clinicienne & $28(63,6)$ & & 4,87 & 0,82 & & 4,15 & 0,81 & & 3,24 & 1,07 & \\
\hline \multicolumn{12}{|l|}{ Statut d'emploi } \\
\hline Temporaire & $13(29,5)$ & 42 & 4,82 & 0,89 & $-0,87$ & 4,04 & 0,92 & $-0,94$ & 3,35 & 1,25 & $-0,22$ \\
\hline Permanent & $31(70,5)$ & & 5,06 & 0,81 & & 4,30 & 0,81 & & 4,43 & 1,03 & \\
\hline \multicolumn{12}{|l|}{ Travail } \\
\hline Temps plein & $31(70,5)$ & 42 & 5,07 & 0,78 & 0,93 & 4,35 & 0,77 & 1,52 & 3,63 & 1,04 & $2,29 *$ \\
\hline Temps partiel & $13(29,5)$ & & 4,81 & 0,96 & & 3,93 & 0,94 & & 2,85 & 1,02 & \\
\hline \multicolumn{12}{|l|}{ Droit de prescrire } \\
\hline Oui & $19(43,2)$ & 42 & 5,20 & 0,85 & 1,42 & 4,63 & 0,80 & $3,04 * *$ & 3,84 & 0,91 & $2,45^{*}$ \\
\hline Non & $25(56,8)$ & & 4,84 & 0,81 & & 3,92 & 0,74 & & 3,08 & 1,11 & \\
\hline \multicolumn{12}{|l|}{ Lieu de travail } \\
\hline Sous-région 1 & $19(43,2)$ & & 5,06 & 0,73 & 170 & 4,24 & 0,69 & & 3,40 & 0,86 & \\
\hline Sous-région 2 & $11(25,0)$ & 2 & 5,27 & 0,92 & 1,70 & 4,57 & 0,92 & 1,95 & 4,04 & 1,22 & $3,74^{*}$ \\
\hline Sous-région 3 & $14(31,8)$ & & 4,68 & 0,86 & & 3,92 & 0,90 & & 2,91 & 1,06 & \\
\hline \multicolumn{12}{|l|}{ Milieu de pratique } \\
\hline CLSC & $23(52,3)$ & & 5,05 & 0,90 & & 4,27 & 0,82 & & 3,49 & 1,10 & \\
\hline$G M F, G M F-U$ & $3(6,8)$ & 3 & 5,14 & 0,71 & 1,19 & 4,23 & 0,42 & 2,00 & 3,41 & 0,61 & 0,88 \\
\hline Dispensaires & $15(34,1)$ & & 4,74 & 0,78 & & 3,96 & 0,83 & & 3,13 & 1,16 & \\
\hline Autres & $3(6,8)$ & & 5,67 & 0,36 & & 5,20 & 0,80 & & 4,19 & 0,80 & \\
\hline \multicolumn{12}{|l|}{ Distance de l'urgence } \\
\hline 30 min. et - & $30(68,2)$ & 42 & 5,09 & 0,82 & 1,13 & 4,34 & 0,82 & 1,37 & 3,56 & 1,02 & 1,34 \\
\hline + de 30 min. ou non relié & $14(31,8)$ & & 4,79 & 0,85 & & 3,97 & 0,86 & & 3,09 & 1,19 & \\
\hline \multicolumn{12}{|l|}{$\begin{array}{l}\text { Collaboration avec } \\
\text { médecin }\end{array}$} \\
\hline médecin & $15(34,1)$ & 42 & 5,17 & 0,74 & 1,02 & 4,45 & 0,83 & 1,28 & 3,57 & 101 & 0,72 \\
\hline Non quotidien & $2965,9)$ & & $\begin{array}{l}0,17 \\
4,90\end{array}$ & 0,88 & & $\begin{array}{l}4,45 \\
4,11\end{array}$ & $\begin{array}{l}0,03 \\
0,84\end{array}$ & & 3,32 & $\begin{array}{l}1,01 \\
1,13\end{array}$ & \\
\hline
\end{tabular}

Note. Le degré de liberté ( $\mathrm{ddl}$ ) désigne le nombre de variables aléatoires qui ne peuvent être déterminées ou fixées par une équation. Celui-ci est égal au nombre d'observations moins le nombre de relations entre ces observations. Les scores établis en fonction $\mathrm{ddl}=42$ ont été établis en fonction de t-test et tandis que pour les autres ddl, les scores ont été établis à l'aide d'ANOVAs. Le tlF désigne la valeur $\mathrm{t}$ pour les t-test et la valeur $\mathrm{F}$ pour les Anovas. Ceux-ci sont exprimés par rapport à la première variable nommée.

$* \mathrm{p}<0,05 \quad *{ }^{*} \mathrm{p}<0,01$ 


\section{CHAPITRE 6}

\section{DISCUSSION}

Ce dernier chapitre présente la discussion des principaux résultats de l'étude où ils sont mis en lien avec les écrits scientifiques. Le chapitre se poursuit avec les limites de l'étude et se termine par une série de recommandations pour la pratique, la formation, la gestion et la recherche.

\subsection{DISCUSSION DES RÉSULTATS SUR LA MESURE DE L'ÉTENDUE EFFECTIVE DE LA PRATIQUE INFIRMIÈRE}

\subsubsection{Score global de l'ÉEPI}

Le score global obtenu dans cette étude $(\mathrm{x}=4,15)$ est satisfaisant, mais laisse tout de même place à l'amélioration. Lorsque comparé à d'autres études, il s'avère que celuici est inférieur à celui mesuré auprès d'infirmières œuvrant dans les services de premières lignes ontariens $(\mathrm{x}=4,81)$ (Braithwaite, 2016) ainsi qu'à celui mesuré en milieu hospitalier d'une région rurale du Liban $(\mathrm{x}=4,42)$ (Younan et al., 2019), similaire à celui mesuré auprès d'infirmières d'un institut universitaire en santé mentale $(\mathrm{x}=4,24)$ (Lafleur, 2016) et plus élevé que d'autres études réalisées en milieu hospitalier ( $\mathrm{x}=3,47)$ (D’Amour et al., 2012) et en pédiatrie ( $x=3,21)$ (Déry, 2013) (Voir tableau 1). Ces scores semblent cohérents avec l'idée générale que le déploiement de la pratique des infirmières est plus grand dans les services de proximité que dans les milieux hospitaliers. 


\subsubsection{Scores pour chaque dimension de l'étendue de la pratique}

La dimension «Évaluation et planification des soins » a obtenu le plus haut score $(\mathrm{x}=4,62)$ qui signifie que ces activités sont fréquemment réalisées. Ce résultat pourrait s'expliquer d'abord par la redéfinition du champ d'exercices des infirmières du Québec en 2003 permettant la reconnaissance par le législateur de leurs compétences et de leurs responsabilités à l'égard de l'évaluation clinique. Celle-ci a récemment conduit à un rehaussement de la formation en évaluation de la condition physique et mentale dans la formation initiale et en cours d'emploi chez l'ensemble des infirmières titulaires d'un diplôme d'études collégiales dans la région à l'étude. Nous pourrions également penser que la norme professionnelle adoptée le 1er avril 2009 par l'OIIQ a elle aussi pu contribuer à accroître ce score en rendant obligatoire la documentation d'un Plan thérapeutique infirmier (PTI). "L'infirmière consigne au dossier de chaque client, dans un outil de documentation distinct, le plan thérapeutique infirmier qu'elle détermine ainsi que les ajustements qu'elle y apporte selon l'évolution clinique du client et l'efficacité des soins et des traitements qu'il reçoit.» (OIIQ, 2016b). Toutefois, il importe de rappeler que rien n'assure dans ces résultats que l'évaluation de la condition physique et mentale et le plan thérapeutique infirmier sont réalisé de façon adéquate. Ce score est cohérent avec les résultats d'une autre étude décrivant la pratique infirmière auprès de personnes atteintes de la maladie d'Alzheimer ou d'autres troubles neurocognitifs majeurs dans des GMF ayant participé aux projets pilotes de la première phase du Plan Alzheimer Québec. Les infirmières participantes avaient alors affirmé que même si elles savaient qu'elles devraient compléter un plan thérapeutique infirmier, elles ne le faisaient pas (NicolClavet, 2017). À l'examen des activités associées à cette dimension, celle qui obtient le score le plus faible est celle liée à la participation à la conception, l'application, l'évaluation et la mise à jour des programmes de soins $(\mathrm{Q} 11=3,14)$. Cette activité est associée au niveau de complexité élevé dont il est attendu qu'elle soit exercée par une infirmière détenant un niveau de formation universitaire. 
Les soins de proximité sont associés à la promotion de la santé et la prévention de la maladie et les infirmières travaillant dans ces milieux sont amenées à adopter des rôles impliquant l'enseignement de la santé comme l'autogestion des maladies chroniques ou l'éducation à la santé sexuelle. Il n'est donc pas étonnant que la dimension «Enseignement à la clientèle et aux familles » obtienne un score satisfaisant $(\mathrm{x}=4,59)$ se plaçant au second rang des dimensions les plus souvent réalisées. Or, une activité de complexité élevée, associée aux activités exercées par l'infirmière clinicienne semble toutefois réalisée moins fréquemment que les autres, soit celle liée à l'évaluation et à l'amélioration de la qualité de l'enseignement dispensé au sein du service (Q22 = 3,57). La dimension «Enseignement à la clientèle et aux familles » obtient également de bons scores dans l'étude sur les services de premières lignes ontariens $(x=5,16)$ (Braithwaite, 2016) ainsi que dans celle menée dans les services de santé mentale en institut universitaire au Québec $(\mathrm{x}=4,46)$ (Lafleur, 2016). Lorsque comparés avec les scores obtenus au sein des autres études, les infirmières en soins de courte durée semblent effectivement se livrer moins fréquemment à ces activités, avec des scores de 3,79 en milieu hospitalier pédiatrique (Déry, 201) et de 3,50 dans les secteurs de médecinechirurgie (D'Amour et al., 2012).

Concernant la dimension «Mise à jour et utilisation des connaissances », le score supérieur à 4,5 pourrait s'expliquer par la norme de formation continue de l'OIIQ qui exige, depuis janvier 2012, que tous ses membres effectuent annuellement au moins 20 heures minimales de formation continue pour renouveler leur permis d'exercice (OIIQ, 2011). L'avènement de la prescription infirmière en 2015, qui est aussi associée à une obligation pour l'infirmière de constamment mettre à jour et développer ses compétences professionnelles (OIIQ et CMQ, 2015), pourrait aussi avoir un impact favorable sur cette dimension de l'ÉEPI. Ce lien est d'ailleurs mis en lumière par Halcomb et al. (2014) dont l'étude a permis d'identifier l'impossibilité de prescrire comme étant une barrière à l'étendue de la pratique des infirmières. Également, il importe de considérer le fait que les infirmières en soins primaires peuvent avoir des conditions facilitantes pour la formation 
continue, par rapport à celles travaillant en milieu hospitalier, en raison de leurs horaires de jour et plus stables et d'un statut d'infirmière clinicienne détenue par la majorité.

Les activités liées à la dimension «Coordination des soins » soient de communiquer, d'établir les responsabilités de chaque membre de l'équipe, de faciliter les transitions en effectuant le transfert des responsabilités et en prodiguant l'information requise et finalement de faire les liens avec les ressources (Radwin, Castonguay, Keenan et Hermann, 2016). En regardant de plus près les activités associées à cette dimension, on constate que la coordination de l'équipe de soins $(\mathrm{Q} 12=3,14)$ et la coordination de l'équipe interprofessionnelle $(\mathrm{Q} 15=3,27)$ obtiennent des scores particulièrement faibles. Ces résultats pourraient indiquer une méconnaissance des infirmières des activités qui doivent être réalisées en coordination des soins ainsi que la présence de défis pour la collaboration interprofessionnelle. Cette dernière va dans le même sens qu'une recherche qualitative réalisée dans les services de première ligne de milieux éloignés au Québec, en 2005 qui avait aussi mis en évidence des enjeux dans la collaboration interprofessionnelle dans ces contextes (Gauthier et al., 2009). Les auteurs avaient alors identifié que le manque de formalisation des liens de collaboration, le roulement du personnel qui affectait les liens de confiance entre les médecins et les infirmières et le mode de rémunération à l'acte des médecins avait tendance à maintenir ces derniers au centre des décisions cliniques et réduire l'autonomie des infirmières (Gauthier et al., 2009). Ceci laisse aussi présager un manque d'expression du leadership clinique des infirmières dans le CISSS à l'étude. Le leadership clinique réfère à l'aptitude de l'infirmière à orienter, à soutenir, à coordonner et à exercer une influence significative sur le patient, sa famille et les autres membres de l'équipe soignante lors de la prestation de soins, et ce même si aucune forme d'autorité officielle ne lui a été dévolue, et ce dans le but d'accomplir les objectifs cliniques partagés par l'équipe de soins (Chàvez \& Yoder, 2015 cités dans Maranda \& Lessard, 2017). En effet, le leadership clinique est étroitement associé à une communication plus efficace entre les membres de l'équipe et à l'amélioration des relations de coordination entre les professionnels de l'équipe de soins (Maranda et Lessard, 2017). 
La dimension «Optimisation de la qualité et de la sécurité des soins » est également étroitement liée à la capacité de leadership clinique des infirmières. En effet, l'amélioration de la qualité des soins de santé requiert le leadership des personnes qui effectuent le travail quotidien (Chàvez et Yoder, 201 528) afin que celles-ci soient en mesure de participer à l'évaluation de la qualité et de la sécurité des soins et d'en signaler les lacunes. Or, cette dimension a obtenu un faible score $(\mathrm{x}=3,82, \sigma=1,14)$ dans le CISSS à l'étude. Les activités les moins souvent réalisées dans cette dimension étaient le développement et l'amélioration de la pratique infirmière $(\mathrm{Q} 3=2,89)$ et l'évaluation de la qualité et de la sécurité des soins $(\mathrm{Q} 23=2,80)$. Ceci peut être tributaire de l'éloignement entre les sites d'exercices où sont offerts les soins primaires et la direction des soins infirmiers qui est responsable de la qualité de l'exercice de la profession. Cela est d'autant plus vrai en contexte de région éloignée où la grandeur du territoire peut constituer une barrière à la présence régulière des conseillères en soins infirmiers dans les services de proximité comparativement à leur présence sur les unités intra hospitalières en raison de la distance ainsi que le nombre et la diversité des lieux de pratique. La diminution des activités assurant la qualité et la sécurité des soins n'est toutefois pas unique à la région à l'étude et serait également associée, selon l'OIIQ (2018), à la détérioration des conditions d'exercice des infirmières au Québec. D'ailleurs, l'organisation, dont la mission première est de protéger le public, sommait récemment le Gouvernement du Québec d'apporter des solutions immédiates et concrètes pour améliorer ces conditions pour améliorer la qualité et la sécurité dans les soins (OIIQ, 2018).

Enfin, la dimension ayant obtenu le plus faible score est "Intégration et encadrement du personnel ». Selon l'Association des infirmières et infirmiers du Canada (AIIC, 2009), dans les services de première ligne, tout comme dans les autres services de santé, le rôle attendu de l'infirmière et l'infirmière clinicienne à l'égard de l'intégration et de l'encadrement du personnel se traduit par l'exercice du leadership infirmier.

Les infirmières de tous les domaines de pratique doivent aider à mettre en place des moyens d'attirer des jeunes, du dynamisme, des idées nouvelles et une énergie nouvelle en leadership des soins infirmiers. [...] Plusieurs stratégies ont 
été décrites dans la littérature pour attirer et retenir les infirmières. Ces stratégies sont inspirées des actions mises en place par les hôpitaux aimants, concept conçu par l'American Academy of Nursing au début des années 1980. Ces établissements se caractérisent notamment par les efforts consentis à la création d'un environnement de travail propice au développement et à l'autonomie professionnelle ainsi qu'à une pratique de soins de qualité. Le soutien clinique est un des moyens faisant partie d'une stratégie globale d'attraction et de rétention. Le soutien clinique est constitué de trois volets, soit l'accueilorientation-intégration, le préceptorat et le mentorat (MSSS, 2008, p.3).

La faible fréquence de réalisation des activités liées à l'intégration et l'encadrement du personnel pourrait notamment s'expliquer par la pénurie de personnel, la surcharge de travail, les difficultés de recrutement, un fort roulement de personnel ainsi que la diminution de personnel d'expérience. Un sondage, réalisé en 2016 par le comité jeunesse de l'OIIQ auprès de la relève infirmière a mis en lumière la présence de difficultés d'intégration chez une infirmière sur trois (Heppell-Cayouette et Maranda, 2016). Les raisons évoquées étaient alors : 1- un accueil quelconque directement reliée à la lourdeur de la tâche, 2- un temps d'orientation et de formation trop court, 3- une difficulté à obtenir des réponses à leurs questions, 4- une difficulté à se faire accepter dans l'équipe de travail et, 5- un manque de soutien (Heppell-Cayouette et Maranda, 2016). Également, certains services de proximité dans lesquels les infirmières sont amenées à effectuer une pratique isolée fait en sorte que l'orientation et la formation de nouveau personnel ne sont pas souvent nécessaires ou à l'opposé, que les nouvelles arrivées sont toujours formées par la même infirmière ce qui diminue la possibilité de formation par les autres infirmières.

Il est intéressant de noter que les résultats par dimension de cette étude diffèrent de ceux des services de proximité de la province voisine (Ontario) où les scores sont plus élevés pour presque toutes les dimensions (Braithwaite, 2016). De plus, la nature des dimensions les plus réalisées diffère. Ainsi, en Ontario, les dimensions « Enseignement à la clientèle et aux familles » et « Mise à jour et l'utilisation des connaissances » obtiennent les scores les plus élevés, tandis que la troisième place était occupée par la dimension de la «Communication et la coordination des soins ». Le contexte des soins primaires ontariens diffère du contexte québécois. En premier lieu, il faut considérer que la présence 
des infirmières au sein des soins primaires sont implantée depuis plus longtemps chez nos voisins ontariens et qu'il est possible que la compréhension du rôle de l'infirmière dans ce contexte soit mieux intégrée qu'au Québec. Enfin, la formation initiale des infirmières présente également des différences puisqu'en Ontario, car l'entrée dans la profession exige un baccalauréat en sciences infirmières.

\subsection{DISCUSSION SUR LES EFFETS DES CARACTÉRISTIQUES INDIVIDUELLES, PROFESSIONNELLES, ORGANISATIONNELLES ET CONTEXTUELLES L'ÉTENDUE DE LA PRATIQUE DES INFIRMIÈRES DES SERVICES DE PROXIMITÉ EN RÉGION ÉLOIGNÉE}

En ce qui concerne les caractéristiques individuelles, professionnelles, organisationnelles et contextuelles, les résultats indiquent que certaines semblent associées à un plus grand déploiement de la pratique infirmière soit : le sexe féminin, le fait de travailler à temps plein, le droit de prescrire ainsi que le lieu de travail à proximité d'un pôle urbain régional. Les différences en fonction du genre ne seront toutefois pas discutées considérant la faible proportion d'hommes dans l'échantillon.

\subsubsection{EEPI et droit de prescrire}

Les résultats obtenus pour le droit de prescrire sont cohérents avec ceux de l'étude de Halcomb et al. (2014) qui ont identifié l'impossibilité de prescrire comme étant une barrière à l'ÉPI.

\subsubsection{EEPI et éloignement}

Le contexte d'éloignement a déjà été mesuré dans l'étude de Birks et al. (2018), toutefois les résultats n'ont pas soulevé de différence significative. 
Il faut toutefois prendre en considération dans la présente étude que les infirmières pratiquant dans les dispensaires des milieux les plus éloignés font face à des défis particuliers et spécifiques liés au contexte d'isolement et au manque de ressources professionnelles. Elles représentent souvent le seul point de contact avec le système de santé et sont en grande partie responsables de la prise en charge des usagers. Cette pratique infirmière au sein des dispensaires isolés est qualifiée de «pratique élargie » ou de « rôle élargi » puisque ces infirmières disposent d'une plus grande autonomie afin d'évaluer les patients, déterminer les suivis cliniques requis et traiter certains problèmes de santé courants selon des guides thérapeutiques et des ordonnances collectives (OIIQ, 2004 ; OIIQ, 2019b). Les infirmières travaillant dans des dispensaires éloignés obtiennent des primes d'infirmière en dispensaire liées à l'exercice d'une pratique en rôle élargi en raison de l'absence de médecin sur place. Dans le cadre de cette étude, il a donc été étonnant de constater que la sous-région 3, associée aux lieux de travail les plus éloignés, ait obtenu des scores plus faibles. Ce résultat est à l'origine d'une réflexion à l'effet que le QÉPI, qui contient peu de questions sur des activités infirmières liées aux interventions directes auprès de la clientèle, ne traduit probablement pas bien la pratique élargie des infirmières des milieux le plus isolés. Combiné au fait que les équipes de soins sont très petites dans ces milieux et que des difficultés d'accès à la formation continue peuvent être présentes en raison de l'absence d'université dans les milieux les plus isolés, cela pourrait influencer à la baisse les résultats obtenus pour l'ÉEPI dans cette sous-région.

\subsubsection{EEPI et formation}

L'étude de Déry (2013) a démontré que le niveau de formation influence l'étendue de la pratique infirmière. Dans la présente étude, l'analyse des données sociodémographiques indique que $56,8 \%$ des infirmières de la présente étude détiennent un baccalauréat en soins infirmiers, 52,6 \% pour l'étude de Lafleur (2016) menée auprès d'infirmières d'un institut universitaire en santé mentale et $62,6 \%$ pour l'étude de 
Braithwaite (2016) en services de première ligne ontariens. Ces taux sont sensiblement plus élevés que le 37,9 \% observé dans l'étude de Déry et al., (2013) et 24,3\% pour l'étude de D’Amours et al. (2012), deux études menées en milieu hospitalier.

Le score moyen global obtenu dans cette étude est inférieur à celui obtenu par Braitwaite (2016) dans les services de première ligne en Ontario indiquant que l'ÉEPI est possiblement moins grande au Québec que dans la province voisine. Ainsi, les infirmières cliniciennes travaillant dans les services de proximité de la région n'arrivent probablement pas à bien déployer leurs acquis universitaires dans leur pratique. Il faut toutefois interpréter ces données avec prudence puisque l'absence de différences peut être tout simplement due à un échantillon trop petit pour la détecter.

\subsubsection{EEPI et expérience de travail}

L'étude de Braithwaite a permis d'établir que l'expérience de travail est une autre caractéristique influençant l'ÉEPI, plus particulièrement la dimension « Intégration et encadrement du personnel », car les infirmières ayant plus de 5 ans d'expérience réalisaient plus fréquemment les activités associées à celle-ci (Braithwaite, 2016). Dans cette étude, les résultats sont mitigés puisque les infirmières détenant plus de 5 ans d'expérience totale de travail réalisent effectivement plus souvent les activités associées à la dimension «Intégration et d'encadrement du personnel » alors que c'est celles détenant moins de 5 ans d'expérience spécifique dans les services de proximité qui semblent réaliser le plus souvent ces activités.

\subsection{LIMITES DE L'ÉTUDE}

Cette étude présente des limites susceptibles d'influencer la validité interne et externe des résultats, telles que la petite taille de l'échantillon et l'utilisation d'une 
méthode d'échantillonnage non probabiliste. Les résultats doivent donc être considérés avec prudence. Si le taux de participation obtenu (33\%) est satisfaisant par rapport au contexte de réalisation de l'étude, ils demeurent nettement en deçà de ceux d'autres études sur l'étendue de la pratique ayant utilisé le QÉPI comme celle de Déry (2013) ayant obtenu un taux de réponse de $90 \%$ en milieu universitaire pédiatrique et celle de Braithwaite (2016) avec un taux de 86,2 \% auprès d'infirmières de services de première ligne en Ontario. Ceci pourrait s'expliquer par l'absence de liste de diffusion ciblée dans le CISSS participant au moment de la recherche et à une tradition de recherche en sciences infirmières en émergence dans la région. Des limites sont aussi associées au questionnaire utilisé. L'équipe a fait le choix d'utiliser un questionnaire ayant déjà été validé dans le cadre d'autres études. Aussi, les modifications apportées dans l'exercice d'adaptation de l'outil ont été réduites au minimum seulement pour mieux tenir compte du contexte des services de proximité. Cependant, il y a lieu de s'interroger si le QÉPI tient compte suffisamment des pratiques dans les milieux plus isolés en raison du peu de questions portant sur les interventions directes des infirmières auprès de la clientèle.

\subsection{RECOMMANDATIONS POUR LA FORMATION, LA PRATIQUE, LA GESTION ET LA RECHERCHE}

Les résultats de cette étude ont mis en lumière certaines pistes pour la recherche, la formation, la pratique infirmière ainsi que la gestion.

\subsubsection{Recommandations pour la formation}

Les résultats de la présente étude démontrent que les infirmières doivent s'approprier davantage leur rôle particulièrement en ce qui concerne les aspects reliés à la dimension concernant l'intégration et l'encadrement du personnel ainsi qu'à l'optimisation de la qualité et la sécurité des soins. À la lumière des présents résultats, il 
serait pertinent de suggérer que la définition de l'étendue de la pratique infirmière (dimensions et niveaux de complexité) ainsi que les effets d'une pratique étendue soient présentés aux étudiants en soins infirmiers, et ce dès le début de leur formation au même titre que le champ de pratique et le code de déontologie. Un cours de soins infirmiers et la communauté ou son équivalent fait déjà partie de la formation universitaire en soins infirmiers. L'ajout de cours spécifique aux soins primaires à l'intérieur du programme de formation au CÉGEP serait également souhaitable afin d'y aborder le rôle de l'infirmière dans ces milieux. Ces connaissances permettraient aux étudiantes infirmières de mieux s'approprier leur rôle en lien avec chacune des dimensions de la pratique infirmière et de mieux comprendre le concept d'étendue de pratique. L'accessibilité aux programmes de formation universitaire en région est donc essentielle afin d'assurer le développement des compétences cliniques chez les infirmières de la relève et celles en pratique (Roy, 2015).

\subsubsection{Recommandations pour la pratique clinique}

Les résultats de cette recherche pourraient également permettre aux infirmières œuvrant dans les services de proximité de mieux comprendre le concept de l'étendue de pratique. Une réflexion s'avère nécessaire sur les raisons expliquant que certaines dimensions soient plus fréquemment réalisées que d'autres. Cette réflexion permettrait aux infirmières de prendre conscience de l'étendue de leur pratique et ainsi voir comment elles peuvent travailler à l'optimiser. Les activités infirmières liées à la coordination de l'équipe de soins et de l'équipe interprofessionnelle sont à développer. Pour ce, il serait intéressant de se pencher sur la notion de leadership clinique afin de s'assurer qu'il s'exprime fortement chez les infirmières, car il favorise ces activités (Maranda \& Lessard, 2017). Il serait également souhaitable de repenser la structure organisationnelle des milieux de soins primaires afin de réduire les facteurs pouvant entraver la coordination des soins tels que la pratique en silo, la gouvernance médicale dans les GMF et GMF-U, etc. Enfin, le soutien clinique des conseillères en soins infirmiers pour les infirmières 
d'expérience et celle de la relève ainsi que davantage de temps dédié aux activités de mentorat et de supervision clinique pourraient contribuer à une meilleure intégration et encadrement du personnel et de formation de la relève pouvant par le fait même accroître l’ÉEPI.

\subsubsection{Recommandations pour la gestion}

La création des CISSS et des CIUSSS présente des défis de gestion importants, notamment pour les infirmières en raison de la distance, des différents contextes et des réalités de pratique variant un peu partout sur le territoire. Les résultats de cette recherche permettent aux gestionnaires des services de proximité de l'établissement de mieux comprendre l'étendue de pratique des infirmières et d'en faciliter un meilleur déploiement dans leurs services de soins primaires. En s'attardant aux différents facteurs d'influence et aux lacunes identifiées au sein des différentes dimensions, les infirmières en situation de gestion et autres gestionnaires pourront identifier des solutions plus concrètes à mettre en place.

Il serait pertinent de revoir l'organisation du travail afin de rendre possible pour l'infirmière clinicienne la mise en œuvre de l'ensemble des activités de soins faisant partie de son étendue de pratique et dont le rôle aurait avantage à être formalisé dans les services de soins primaires. En ce sens, Déry (2013) écrit :

En ce sens, il appert crucial de réfléchir aux conditions dans lesquelles le rôle d'infirmière clinicienne s'est implanté depuis 2005 dans les organisations de soins. Il semble que cette implantation se soit faite sans qu'elle soit nécessairement accompagnée d'une démarche de changement organisationnel. Tel que mentionné par Smith, Manfredi, Hagos, Drummond-Huth, et Moore, (2006), qui se sont intéressés à l'introduction du rôle de la «Clinician Nurse Leader », un rôle similaire au titre d'infirmière clinicienne, l'implantation d'un nouveau poste nécessite l'implication de tous les membres de l'équipe de soins. Une redéfinition du rôle de chacun des membres de l'équipe de soins doit également accompagner cette implantation (Smith et al., 2006) en fonction de la valeur ajoutée du nouveau rôle au sein de l'équipe (Déry, 2013, p.140). 
Il appert nécessaire de travailler à accroître l'expression du leadership clinique des infirmières en créant de nouvelles cultures de leadership au sein des organisations (Maranda et Lessard, 2017) et en misant sur le développement et le renforcement continus des compétences favorisant l'expression du leadership clinique chez les infirmières de la relève et celles en pratique (Roy, 2015). Ces solutions passent par l'accès à des formations continues permettant le développement de ces compétences (Roy, 2015 ; Maranda et Lessard, 2017), l'instauration des programmes de mentorat ou de supervision clinique (Roy, 2015 ; Maranda et Lessard, 2017) et la mise en place d'une gouvernance clinique partagée favorisant l'implication active des infirmières (Roy, 2015). À cet effet, une présence plus soutenue de la direction des soins infirmiers et des conseillères en soins infirmiers dans les services de proximité pourrait contribuer à favoriser le développement des compétences infirmières en plus d'assurer un soutien clinique à l'ensemble des infirmières ouvrant dans ces contextes.

Il est également important de travailler à améliorer la collaboration interprofessionnelle par une plus grande formalisation des liens (Lessard, Morin et Sylvain, 2008 ; Careau, Brière, Houle, Dumont, Maziades, Paré et al., 2014) : 1- en permettant une meilleure compréhension de la contribution des différents membres de l'équipe de soins, dont les infirmières, par une définition claire de leurs rôles et responsabilités ; 2- en favorisant le déploiement de pratiques collaboratives à large échelle ; 3- en améliorant le partage d'information et la communication entre les professionnels (Roy, 2015) et ; 4- en travaillant le rôle de coordination des infirmières en soins primaires.

Finalement, l'amélioration globale des conditions de pratique des infirmières est susceptible d'améliorer les activités liées à l'évaluation de la qualité et de la sécurité des soins et contribuer à une plus grande ÉEPI (Radwin et al., 2016). 


\subsubsection{Recommandations pour la recherche}

Plusieurs recherches ont démontré que les infirmières ne parviennent pas à mettre en ouvre l'ensemble des activités pour lesquelles elles détiennent la formation et l'expérience (Besner et al., 2005; D’Amour et al., 2012; Oelke et al., 2008; White et al., 2008). Peu d'études se sont attardées à l'étendue de pratique des infirmières dans les services de proximité et aucune n'a mesuré de façon quantitative cette étendue de pratique dans une région éloignée du Québec. Ce mémoire a tenté de combler ce manque de connaissance en mesurant l'ÉEPI dans les services de proximité d'une région éloignée en plus d'accroître le corpus de connaissance en lien avec l'ÉEPI. Les résultats de cette étude suscitent des réflexions sur l'ÉEPI et permettent d'orienter l'action afin de favoriser un plus grand déploiement de la pratique infirmière par les recommandations qui y sont présentées. Il serait donc intéressant de mesurer à nouveau l'ÉEPI après la mise en place de stratégies visant à favoriser un meilleur déploiement des activités mises en œuvre par les infirmières et les infirmières cliniciennes. Il serait également bénéfique que des travaux soient réalisés pour en créer une version adaptée à ces contextes particuliers. Aussi, il serait pertinent, dans de futures recherches, de prendre en considération la réalisation de l'évaluation de la condition physique et mentale et le plan thérapeutique infirmier afin de s'assurer que ceux-ci sont réalisés de façon adéquate. Finalement, le portait régional demeure partiel puisque les infirmières travaillant dans les centres de santé autochtones de la région n'ont pas été incluses dans l'étude. D'autres caractéristiques individuelles, professionnelles, organisationnelles et contextuelles pouvant influencer l'ÉEPI que celles évaluées dans cette étude pourraient être examinées dans de futures recherches comme le niveau de leadership clinique chez les infirmières et le niveau de collaboration interprofessionnelle. 


\section{CONCLUSION GÉNÉRALE}

La pratique des infirmières travaillant dans les services de proximité en région estelle optimale pour leur permettre de bien contribuer à améliorer l'accessibilité et la continuité des soins et services en région? Tout d'abord, les résultats laissent penser que le rôle d'infirmière clinicienne n'est pas déployé de manière optimale au sein des services de proximité. Le but de cette recherche était d'établir un portrait de l'étendue de la pratique des infirmières travaillant dans les services de proximité dans une région éloignée du Québec. Celle-ci a mis en évidence certains manques à gagner dans la pratique des infirmières exerçant dans les services de proximité d'une région éloignée du Québec et a identifié certaines caractéristiques pouvant augmenter l'ÉEPI dans ce contexte, soit le sexe féminin, le temps de travail à temps plein, le lieu de travail à proximité d'un pôle urbain régional et le droit de prescrire.

Elle a permis de cibler des forces dans la pratique infirmière de ces milieux, dont plusieurs activités associées aux dimensions de l'évaluation et la planification des soins, de l'enseignement à la clientèle et aux familles, ainsi que celle de la mise à jour et l'utilisation des connaissances. Des lacunes ont également été observées dans les dimensions liées à la communication et la coordination des soins, à l'optimisation de la qualité et de la sécurité des soins et à l'intégration et l'encadrement du personnel. Ces résultats renforcent le besoin de porter une attention particulière aux compétences de leadership clinique et de coordination des soins chez les infirmières. D'ailleurs, le ministère de la Santé et des Services sociaux du Québec souligne que «l'implication des infirmières comme acteurs majeurs dans des réseaux intégrés de soins exige d'elles de développer un ensemble de compétences clés qui sont de trois ordres : des compétences cliniques, des compétences en matière de coordination des soins et des compétences de leadership » (MSSS, 2013, p.16).

Cette étude contribue à l'avancement des sciences infirmières en accroissant le corpus de connaissances en lien avec l'ÉEPI et les caractéristiques l'influençant en région 
éloignée. Elle est l'une des premières à s'intéresser à l'étendue effective de la pratique des infirmières dans les services de proximité dans une région éloignée en contexte québécois.

Les résultats de cette étude constituent un levier important pour recommander des actions concrètes visant à optimiser le déploiement de la pratique professionnelle des infirmières dans les contextes de soins de proximité qui pourrait permettre d'accroître l'accessibilité aux services de santé, la qualité et la sécurité des soins ainsi que la satisfaction de la clientèle envers les soins et services reçus. Des retombées positives sont également prévisibles pour les infirmières dont une plus grande satisfaction professionnelle et pour les organisations avec une hausse d'efficacité et une performance accrue. Cette étude est une contribution empirique permettant de mieux comprendre la pratique infirmière dans les services de proximité et met en lumière les défis de cette pratique dans une région éloignée du Québec. 
APPENDICE I

Soumission de l'article - Revue RSI 


$\begin{array}{ll}\text { Date: } & 18 / 09 / 2019 \\ \text { À: } & \text { "Mélanie Morin" m.morin1981@hotmail.com } \\ \text { De: } & \text { "Recherche en Soins Infirmiers" noreply@asso-arsi.fr } \\ \text { Objet: } & \text { Réception des révisions pour RSI0143R2 }\end{array}$

Ref.: Ms. No. RSI0143R2

L'étendue effective de la pratique des infirmières dans les services de proximité en région éloignée.

Madame, Monsieur,

Nous accusons réception de la nouvelle version de votre article ainsi que de vos réponses aux évaluateurs et nous vous en remercions.

Nous soumettons ces documents à notre comité scientifique et reviendrons vers vous dès que possible.

En vous remerciant pour l'intérêt que vous portez à la revue Recherche en soins infirmiers.

Cordialement

Conformément aux réglementations sur la protection des données, vous pouvez demander à tout moment la suppression de vos informations personnelles d'inscription. (Utilisez l'URL suivante : https://www.editorialmanager.com/rsi/login.asp?a=r) Pour toute question, contactez le bureau de la revue. 


$\begin{array}{ll}\text { Date: } & 18 / 09 / 2019 \\ \text { À: } & \text { "Mélanie Morin" m.morin1981@hotmail.com } \\ \text { cc: } & \text { "Lily Lessard" lily_lessard@uqar.ca } \\ \text { De: } & \text { "Recherche en Soins Infirmiers" noreply@asso-arsi.fr } \\ \text { Objet: } & \text { Votre soumission }\end{array}$

Ref.: Ms. No. RSI0143R2

L'étendue effective de la pratique des infirmières dans les services de proximité en région éloignée. Recherche en soins infirmiers

Chère Madame Morin,

je vous remercie d'avoir révisé votre article et répondu aux commentaires dans une période chargée de travail pour vous.

Nous avons le plaisir de vous annoncer que votre article intitulé * L'étendue effective de la pratique des infirmières dans les services de proximité en région éloignée. » est accepté pour publication dans la revue Recherche en soins infirmiers.

Nous reviendrons vers vous pour relecture et validation de la version finale avant publication dans le prochaine numéro de RSI.

Cordialement

Emmanuelle Cartron,

Pour la rédaction

Recherche en soins infirmiers

Commentaires :

Conformément aux réglementations sur la protection des données, vous pouvez demander à tout moment la suppression de vos informations personnelles d'inscription. (Utilisez l'URL suivante :

https://www.editorialmanager.com/rsi/login.asp?a=r) Pour toute question, contactez le bureau de la revue. 
APPENDICE II

Activités infirmières réservées des services de proximité (OIIQ, 2014 b, 2016) 


\begin{tabular}{l} 
Les 17 activités réservées \\
\hline 1. Évaluer la condition \\
physique et mentale d'une \\
personne symptomatique; \\
\\
\\
thérapeutique infirmier; \\
personnes, dont l'état de \\
santé présente des risques, \\
compris le monitorage et \\
ajustements du plan
\end{tabular}

Les éléments du champ d'exercices appliqués aux soins de proximité

Procéder à l'évaluation initiale à la clinique sans rendez-vous et déterminer le degré de gravité et d'urgence du problème de santé physique.

Effectuer l'examen clinique du nourrisson.

Évaluer l'état nutritionnel d'un client qui présente une plaie récalcitrante.

Évaluer les habitudes de vie d'un client qui débute un nouvel antipsychotique atypique.

Analyser les facteurs de risque cardiovasculaire d'un client.

Évaluer la capacité du client et de sa famille à effectuer les autosoins dont il a besoin et à prendre en charge sa santé.

Évaluer la croissance, le stade de développement de la personne selon son âge et déterminer les écarts.

Évaluer le niveau d'autonomie et les déficits cognitifs d'une personne âgée à domicile, en utilisant des outils de dépistage et d'évaluation validés.

Détecter les situations potentielles de violence conjugale, lors de la première visite de la femme enceinte à la clinique prénatale du CLSC.

Évaluer le risque suicidaire et la dangerosité d'un passage à l'acte suicidaire.

Procéder au suivi périodique de l'enfant.

Effectuer le monitorage maternel et fotal d'une femme présentant une grossesse à risque en suivi à domicile.

Effectuer la surveillance d'un client diabétique à l'aide d'un système de télésurveillance à distance.

Transmettre à un client, qui contacte Info-Santé, les éléments de surveillance relatifs à son problème de santé et les indications de consulter, au besoin.

Coordonner la référence d'un client vers la 2e ligne, et assurer la surveillance clinique jusqu'à la consultation.

Donner des directives cliniques au PTI concernant les éléments de surveillance à mettre en place pour le suivi d'un enfant qui reçoit des gavages à domicile.

Déterminer et ajuster le PTI pour assurer la surveillance clinique requise.

3. Initier des mesures
diagnostiques
thérapeutiques, selon une
ordonnance;
ordonnance;
Initier des analyses (E+, créatinine, glycémie, bilan lipidique) dans le cadre du suivi d'un client hypertendu.

Initier un prélèvement de RNI chez un client anticoagulé.

Initier un traitement du muguet chez une mère qui allaite et son nouveau-né.

Initier la contraception orale d'urgence.

Initier la contraception hormonale.

Initier la nitroglycérine sublinguale chez un client avec des douleurs rétro sternales

\footnotetext{
4. Initier des mesures diagnostiques à des fins de dépistage, dans le cadre d'une activité découlant de l'application de la Loi sur la santé publique;
}

Dépister la présence de l'infection à la gonorrhée chez une femme de moins de 25 ans avant l'installation d'un stérilet.

Dépister les ITSS chez une adolescente présentant des facteurs de risque, à la clinique jeunesse.

Initier dépistage sanguin pour l'hépatite $\mathrm{C}$ chez utilisateur de drogues injectables. 


\section{Effectuer des examens et des tests diagnostiques invasifs, selon une ordonnance;}

Effectuer : une cytologie cervicale, un test de réactivité fotale, les examens diagnostiques à des fins sérologiques, biochimiques ou hématologiques.

Effectuer une échographie gestationnelle lors d'une interruption volontaire de grossesse.

Effectuer les tests de fonction pulmonaire (spirométrie et débit expiratoire de pointe). $•$ Effectuer un audiogramme dans le cadre d'un programme en santé et sécurité au travail.

\section{Effectuer et ajuster les traitements médicaux, selon une ordonnance;}

\section{Administrer les injections de désensibilisation chez un client allergique.}

Procéder aux traitements des verrues avec la cryothérapie.

Procéder à l'immobilisation d'un membre suite à une fracture à l'unité de médecine familiale.

Procéder à une irrigation oculaire suite à une exposition à un produit chimique.

Ajuster le débit et la fréquence de l'oxygénothérapie à domicile, selon la réaction du client au traitement.

Retirer le stérilet en GMF.
7. Déterminer le plan de traitement relié aux plaies et aux altérations de la peau et des téguments et prodiguer les soins et les traitements qui s'y rattachent;
Procéder au nettoyage de la plaie.

Procéder au débridement de la plaie.

Déterminer les produits et pansements appropriés à la plaie.

Procéder à la fermeture d'une plaie à l'aide des diachylons de rapprochement.

Enlever la mèche installée dans une plaie.

Décider d'appliquer des modalités de compression dans le cas d'ulcère veineux ou lymphatique.

Traiter une brûlure aux services courants d'un CLSC et en déterminer le suivi.

Effectuer les soins de stomie en post-chirurgie à domicile.

\section{Appliquer techniques invasives; \\ des \\ 9. Contribuer au suivi de la grossesse, à la pratique des accouchements et au suivi postnatal;}

Effectuer le toucher du col utérin chez une femme enceinte.

Effectuer une infiltration de cortisone dans la bourse sous-acromiale.

Insérer un spéculum pour l'examen visuel du col utérin.

Retirer un cathéter central inséré par voie périphérique.

\section{Donner des classes prénatales.}

Effectuer le counseling pour la planification familiale.

Effectuer une évaluation préconceptionnelle.

Assurer le suivi de grossesse en collaboration avec un médecin.

Gérer les malaises courants de la grossesse.

Détecter les complications pouvant nuire au bien-être maternel et foetal.

Évaluer et surveiller le développement fotal.

Examiner le nouveau-né et la nouvelle accouchée.

Surveiller l'état de santé et la tolérance alimentaire du nouveau-né.

Soutenir l'allaitement maternel.

Évaluer l'adaptation parentale.

Détecter des situations de négligence ou d'abus chez l'enfant.

Évaluer les signes de la présence possible d'un ictère chez le nouveau-né et mesurer le taux de bilirubine 


10. Effectuer le suivi
infirmier des personnes
présentant des problèmes
de santé complexes;
de santé complexes;
Effectuer le suivi clinique de maladies chroniques (diabète, maladie pulmonaire obstructive, insuffisance cardiaque, hypertension artérielle, dépression, etc.) en collaboration avec d'autres professionnels ou services.

Enseigner au client et à sa famille comment reconnaître et gérer les signes et symptômes de détérioration respiratoire de l'enfant asthmatique.

Faire la promotion de saines habitudes de vie en utilisant des approches reconnues, dont l'approche motivationnelle.

Procéder au suivi systématique de clientèle postopératoire à domicile.

Vérifier la réponse du client au traitement, en effectuant certaines analyses de laboratoire, selon ordonnance collective (ex. protocole de surveillance clozapine).

Gérer les symptômes et la douleur du client en fin de vie en soins à domicile.

Agir comme gestionnaire de cas et coordonner les services intégrés et concertés pour les grands utilisateurs de service.

Déterminer et ajuster le PTI pour assurer le suivi clinique requis.

11. Administrer et ajuster
des médicaments ou
d'autres substances,
lorsqu'ils font l'objet d'une
ordonnance;
ordonnance;

Évaluer le profil médicamenteux d'un client à domicile incluant les médicaments en vente libre et les produits naturels, s'assurer de sa mise à jour et exercer une pharmacovigilance pour une utilisation optimale des médicaments.

Observer et documenter les effets thérapeutiques des médicaments prescrits.

Effectuer le suivi d'un client sous anticoagulant et ajuster la médication selon le résultat du RNI.

Ajuster l'insuline selon la réponse thérapeutique du client.

12. Procéder à la vaccination, dans le cadre d'une activité découlant de l'application de la Loi sur la santé publique;
Évaluer le statut vaccinal d'un enfant, décider de vacciner au besoin avec le consentement des parents.

Effectuer la prophylaxie en cas de blessure aux services courants du CLSC.

Procéder à la vaccination du voyageur et au counseling.

Effectuer un test à la tuberculine(TCT) aux étudiants en soins infirmiers.

Effectuer la primo-vaccination des nourrissons.

Mener les campagnes massives de vaccination antigrippale.

\section{Mélanger des substances en vue de compléter la préparation d'un médicament, selon une ordonnance;}

14. Décider de l'utilisation Décider exceptionnellement d'utiliser une mesure de contrôle au domicile d'un des mesures de contention; client en attendant l'ambulance.

Décider d'utiliser une mesure temporaire d'isolement pour enfant dans un camp de vacances, si son comportement met la sécurité des autres enfants en danger.

\footnotetext{
15. Décider de l'utilisation des mesures d'isolement dans le cadre de l'application de la Loi sur les services de santé et les services sociaux et de la Loi sur les services de santé et les services sociaux pour les autochtones cris;

16. Évaluer les troubles mentaux, à l'exception du retard mental, lorsque l'infirmière ou l'infirmier détient une formation de niveau universitaire et une expérience clinique en soins infirmiers psychiatriques, déterminées dans le cadre d'un règlement pris en application du paragraphe g de l'article 14;
} 


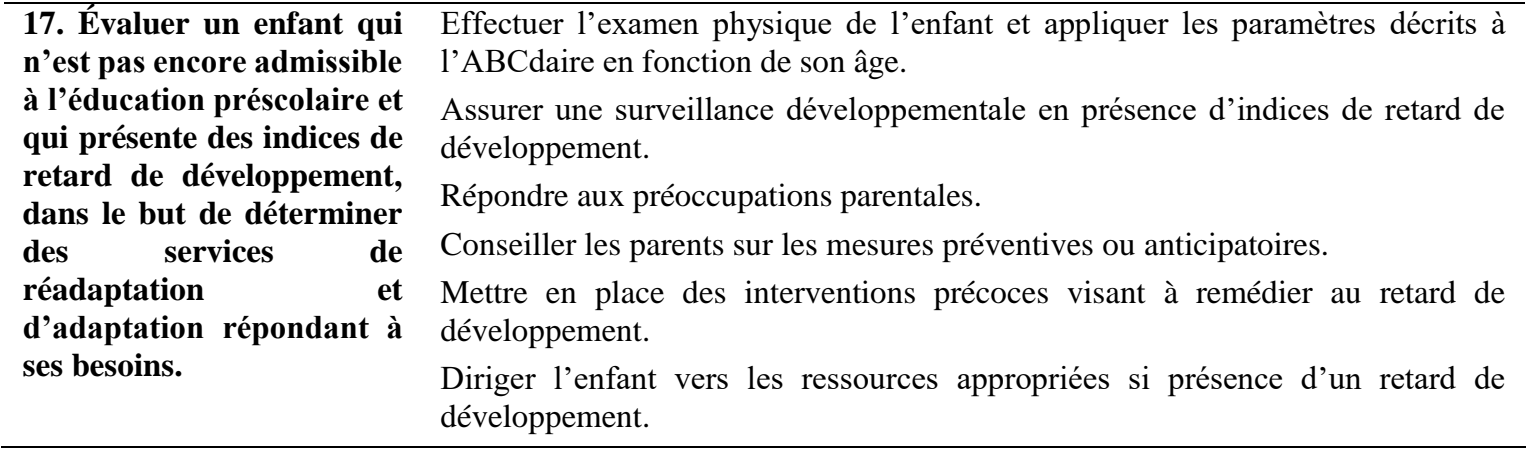


APPENDICE III

QÉPI instrument version 2011 


\section{PRATIQUE INFIRMIÈRE}

Ce questionnaire vise à mieux connaître la réalité de votre pratique dans votre unité, en tenant compte du fait que différentes contraintes peuvent l'influencer.

Instructions : Pour chaque énoncé, remplir la bulleréponse correspondant le mieux à votre pratique quotidienne dans votre unité.

Si pour une raison ou une autre, un énoncé ne s'applique pas à votre situation, remplir la bulleréponse 1 signifiant "Jamais».

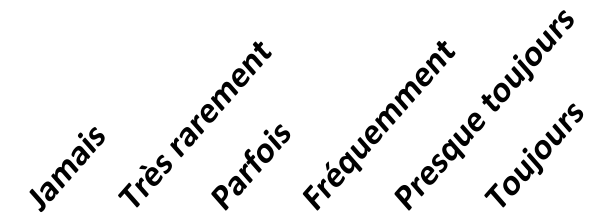

$\begin{array}{lllllllll}\text { 1. Je suscite l'implication du client et de sa famille } & \mathbf{1} & \mathbf{2} & \mathbf{3} & \mathbf{4} & \mathbf{5} & \mathbf{6}\end{array}$ dans la planification des soins.

2. Pour planifier mes interventions, j'utilise des outils d'évaluation de problèmes de soins (ex. : échelle d'évaluation de la douleur, outil d'évaluation des plaies, outil d'évaluation pour les contentions).

3. Je participe au développement de la pratique infirmière (ex. révision de protocoles de soins, projets divers)

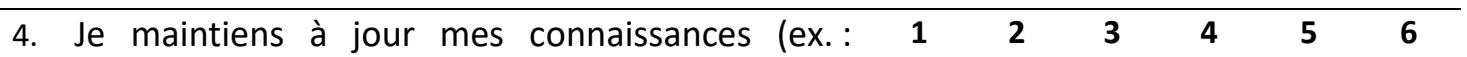
lectures, activités scientifiques).

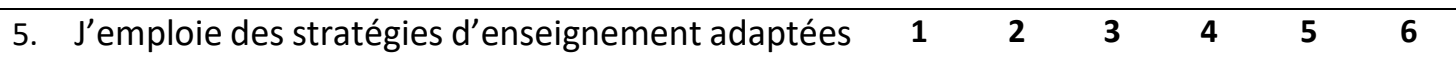
à chaque client et à sa famille, en fonction du degré d'autonomie du client.

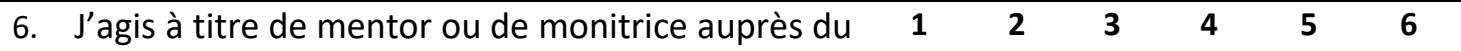
personnel nouvellement recruté. 
\begin{tabular}{lllllllll}
\hline 7. Je signale les situations cliniques où je perçois une & $\mathbf{1}$ & $\mathbf{2}$ & $\mathbf{3}$ & $\mathbf{4}$ & $\mathbf{5}$ & $\mathbf{6}$
\end{tabular} lacune dans la qualité et la sécurité des soins.

$\begin{array}{lllllllll}\text { 8. Je participe aux réunions ou à des activités de } & \mathbf{1} & \mathbf{2} & \mathbf{3} & \mathbf{4} & \mathbf{5} & \mathbf{6}\end{array}$ l'équipe interprofessionnelle (médecin, physiothérapeute, etc.)

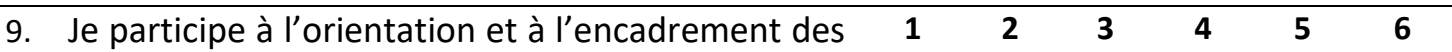
stagiaires ou du personnel nouvellement recruté.

10. J'évalue les besoins spécifiques d'information et $\quad \begin{array}{lllllll}\mathbf{1} & \mathbf{2} & \mathbf{3} & \mathbf{4} & \mathbf{5} & \mathbf{6}\end{array}$ d'enseignement propres à chaque client et à sa famille.

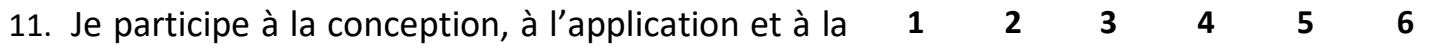
mise à jour des programmes de soins (ex. : un suivi systématique de clientèle, des plans de soins standardisés, un suivi intégré).

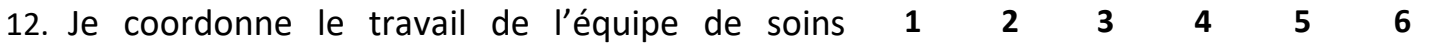
infirmiers (infirmière, infirmière auxiliaire, préposé aux bénéficiaires) pour répondre aux besoins du client et de sa famille.

13. Lors d'une modification de la pratique, je partage $\begin{array}{llllll}1 & 2 & 3 & 4 & 5 & 6\end{array}$ avec l'équipe de soins infirmiers les connaissances issues de la recherche.

14. Je participe au développement ou à la dispensation d'activités de formation à l'équipe de soins, selon mes compétences.

15. Afin d'assurer la continuité des soins, je coordonne les interventions de l'équipe interprofessionnelle au sein de l'établissement (médecin, physiothérapeute, etc.)

16. Je communique aux membres de l'équipe toute $\quad \begin{array}{llllllll}\mathbf{1} & \mathbf{2} & \mathbf{3} & \mathbf{4} & \mathbf{5} & \mathbf{6}\end{array}$ information pertinente susceptible d'influencer la coordination des soins.

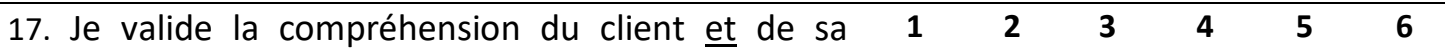
famille par rapport à l'enseignement reçu. 


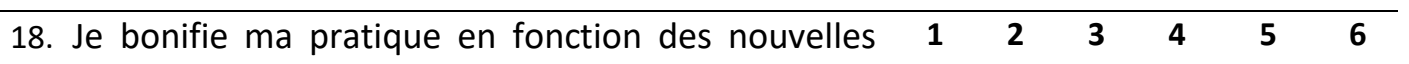
connaissances issues des pratiques exemplaires et des projets de recherche en sciences infirmières ou en santé.

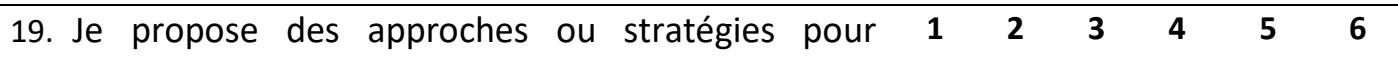
améliorer la qualité et la sécurité des soins lorsque j'identifie des lacunes.

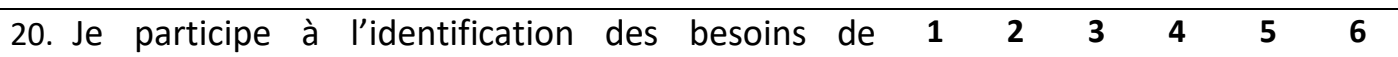
formation de mon unité.

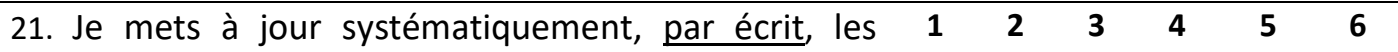
informations sur la condition du client et sur les soins dispensés (notes de l'infirmière, plan thérapeutique infirmier, etc.)

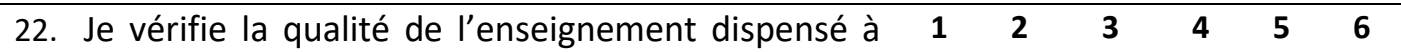
l'unité.

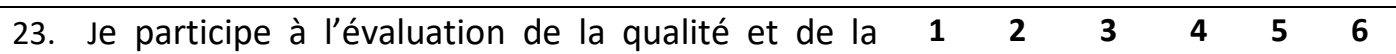
sécurité des soins (utilisation d'outils ou de systèmes d'évaluation de la qualité des soins, questionnaire de satisfaction, etc.).

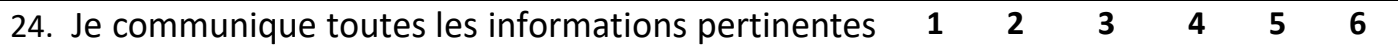
aux professionnels d'autres établissements en vue d'assurer la continuité des soins.

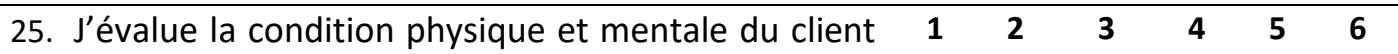
en considérant les dimensions biopsychosociales.

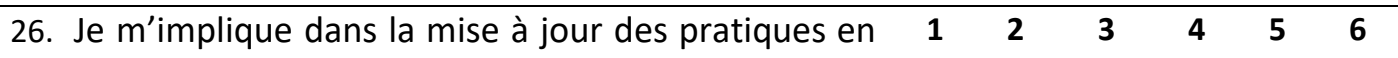
vue d'améliorer la qualité et la sécurité des soins. 
APPENDICE IV

Adaptation du QÉPI 
Adaptation du QÉPI et Alpha de cronbach (consistance interne) du score global de l'ÉEPI, de ses dimensions et niveaux de complexité selon les différentes études

\begin{tabular}{|c|c|c|c|c|c|c|}
\hline \multicolumn{4}{|c|}{ Questionnaire de l'étendue de la pratique infirmière (QÉPI) } & \multicolumn{3}{|c|}{ Alpha de cronbach (consistance interne) } \\
\hline & QÉPI original (D’Amours et al., 2012) & $\begin{array}{l}\text { Adaptation au contexte de soins de } \\
\text { proximité québécois par cette étude }\end{array}$ & $\begin{array}{l}\text { Adaptation au contexte de soins } \\
\text { primaires (Braithwaite, 2016) }\end{array}$ & $\begin{array}{c}\text { D'Amours et al. } \\
\text { (2012) }\end{array}$ & $\begin{array}{l}\text { Cette } \\
\text { étude }\end{array}$ & $\begin{array}{c}\text { Braithwaite, E. } \\
\text { (2016) }\end{array}$ \\
\hline \multicolumn{4}{|c|}{ Dimension 1 : Évaluation et planification des soins } & 0,64 & 0,68 & 0,67 \\
\hline Q1 & $\begin{array}{l}\text { Je suscite l'implication du client et de sa } \\
\text { famille dans la planification des soins. }\end{array}$ & $\begin{array}{l}\text { Je planifie les soins du client en } \\
\text { collaboration avec le client et sa famille. } \\
\text { (Mots-clés : planification soins) }\end{array}$ & $\begin{array}{l}\text { Je développe des plans de soins aux } \\
\text { patients en collaboration avec le patient, } \\
\text { la famille du patient et l'équipe de soins } \\
\text { interprofessionnels. }\end{array}$ & & & \\
\hline Q2 & $\begin{array}{l}\text { Pour planifier mes interventions, j'utilise } \\
\text { des outils d'évaluation de problèmes de } \\
\text { soins (ex.: échelle d'évaluation de la } \\
\text { douleur, outil d'évaluation des plaies, } \\
\text { outil d'évaluation pour les contentions). }\end{array}$ & $\begin{array}{l}\text { Pour planifier mes interventions, j'utilise } \\
\text { des lignes directrices sur les meilleures } \\
\text { pratiques et des outils d'évaluation } \\
\text { fondés sur des données probantes } \\
\text { (échelles de douleur, outils d'évaluation } \\
\text { des plaies...) } \\
\text { (Mots-clés: utilisation outils } \\
\text { d'évaluation) }\end{array}$ & $\begin{array}{l}\text { J'utilise des lignes directrices sur les } \\
\text { meilleures pratiques et des outils } \\
\text { d'évaluation fondés sur des données } \\
\text { probantes (échelles de douleur, outils } \\
\text { d'évaluation des plaies, etc.) pour } \\
\text { planifier les interventions infirmières en } \\
\text { collaboration avec les patients et leur } \\
\text { famille. }\end{array}$ & & & \\
\hline Q11 & $\begin{array}{l}\text { Je participe à la conception, à } \\
\text { l'application et à la mise à jour des } \\
\text { programmes de soins (ex.: un suivi } \\
\text { systématique de clientèle, des plans de } \\
\text { soins standardisés, un suivi intégré). }\end{array}$ & $\begin{array}{l}\text { Je participe à la conception, l'application, } \\
\text { l'évaluation et la mise à jour des } \\
\text { programmes de soins aux clients (ex. } \\
\text { suivi systématique de clientèle, plans de } \\
\text { soins standardisés, suivi intégré). } \\
\text { (Mots-clés: conception et mises à jour } \\
\text { programmes de soins) }\end{array}$ & $\begin{array}{l}\text { Je suis impliqué dans la conception, } \\
\text { l'application et } \\
\text { l'évaluation/l'amélioration des } \\
\text { programmes de soins aux patients. }\end{array}$ & & & \\
\hline Q21 & $\begin{array}{l}\text { Je mets à jour systématiquement, par } \\
\text { écrit, les informations sur la condition du } \\
\text { client et sur les soins dispensés (notes de } \\
\text { l'infirmière, plan thérapeutique } \\
\text { infirmier, etc.) }\end{array}$ & $\begin{array}{l}\text { Je mets à jour, systématiquement et par } \\
\text { écrit, les informations sur la condition du } \\
\text { client (évaluation infirmière) et sur les } \\
\text { soins dispensés (notes de l'infirmière, } \\
\text { plan thérapeutique infirmier, etc.) } \\
\text { (Mots-clés: documentation régulière de } \\
\text { la condition du client) }\end{array}$ & $\begin{array}{l}\text { Je documente l'information sur l'état du } \\
\text { patient (évaluation infirmière) et les } \\
\text { soins fournis (plan thérapeutique } \\
\text { infirmier, notes infirmières, etc.). }\end{array}$ & & & \\
\hline
\end{tabular}




\begin{tabular}{|c|c|c|c|c|c|c|}
\hline Q25 & $\begin{array}{l}\text { J'évalue la condition physique et mentale } \\
\text { du client en considérant les dimensions } \\
\text { biopsychosociales. }\end{array}$ & $\begin{array}{l}\text { J'évalue de manière autonome la } \\
\text { condition physique et mentale du client } \\
\text { en considérant les dimensions } \\
\text { biopsychosociales. } \\
\text { (Mots-clés : évaluation autonome de la } \\
\text { condition) }\end{array}$ & $\begin{array}{l}\text { J'évalue de manière autonome l'état } \\
\text { physique et mental du patient en tenant } \\
\text { compte des aspects biopsychosociaux. }\end{array}$ & & & \\
\hline Q27 & & & $\begin{array}{l}\text { J'interprète les résultats de l'évaluation, } \\
\text { coordonne/met en œuvre d'autres } \\
\text { actions infirmières et coordonne les } \\
\text { actions requises par les autres membres } \\
\text { de l'équipe soignante du patient. }\end{array}$ & & & \\
\hline \multicolumn{4}{|c|}{ Dimension 2 : Enseignement à la clientèle et aux familles } & 0,67 & 0,764 & 0,64 \\
\hline Q5 & $\begin{array}{l}\text { J'emploie des stratégies d'enseignement } \\
\text { adaptées à chaque client et à sa famille, } \\
\text { en fonction du degré d'autonomie du } \\
\text { client. }\end{array}$ & $\begin{array}{l}\text { J'utilise des stratégies d'enseignement } \\
\text { adaptées à chaque client et à sa famille } \\
\text { en fonction des besoins actuels du client } \\
\text { et de sa famille et de leur degré } \\
\text { d'autonomie. } \\
\text { (Mots-clés: stratégies enseignement } \\
\text { adaptées) }\end{array}$ & $\begin{array}{l}\text { J'utilise des stratégies d'enseignement } \\
\text { adaptées à chaque patient et à chaque } \\
\text { famille en fonction des besoins actuels } \\
\text { du patient, de sa compétence } \\
\text { intellectuelle, de son stade de } \\
\text { développement, de son niveau } \\
\text { d'alphabétisation, etc. }\end{array}$ & & & \\
\hline Q10 & $\begin{array}{l}\text { J'évalue les besoins spécifiques } \\
\text { d'information et d'enseignement } \\
\text { propres à chaque client et à sa famille. }\end{array}$ & $\begin{array}{lrr}\text { J'évalue les } & \text { besoins } & \text { spécifiques } \\
\text { d'information } & \text { et d'enseignement } \\
\text { propres à chaque client et à sa famille. } \\
\text { (Mots-clés : } & \text { évaluer } & \text { besoins } \\
\text { d'information) } & & \end{array}$ & $\begin{array}{l}\text { J'évalue les besoins en éducation de } \\
\text { chaque patient et de sa famille. }\end{array}$ & & & \\
\hline Q17 & $\begin{array}{l}\text { Je valide la compréhension du client et } \\
\text { de sa famille par rapport à } \\
\text { l'enseignement reçu. }\end{array}$ & $\begin{array}{l}\text { J'évalue la compréhension de } \\
\text { l'enseignement dispensé au client et à sa } \\
\text { famille et adapte les interventions } \\
\text { éducatives en conséquence. } \\
\text { (Mots-clés: adaptation des } \\
\text { interventions éducatives) }\end{array}$ & $\begin{array}{l}\text { J'évalue la compréhension de } \\
\text { l'enseignement dispensé au patient et à } \\
\text { sa famille et adapte les interventions } \\
\text { éducatives en conséquence. }\end{array}$ & & & \\
\hline Q22 & $\begin{array}{l}\text { Je vérifie la qualité de l'enseignement } \\
\text { dispensé à l'unité. }\end{array}$ & $\begin{array}{l}\text { J'évalue et améliore lorsque nécessaire } \\
\text { la qualité de l'enseignement dispensé au } \\
\text { sein du service. } \\
\text { (Mots-clés: évaluation et amélioration } \\
\text { enseignement) }\end{array}$ & $\begin{array}{l}\text { J'évalue et améliore (le cas échéant) la } \\
\text { qualité de l'éducation des patients } \\
\text { fournie dans mon organisation. }\end{array}$ & & & \\
\hline
\end{tabular}




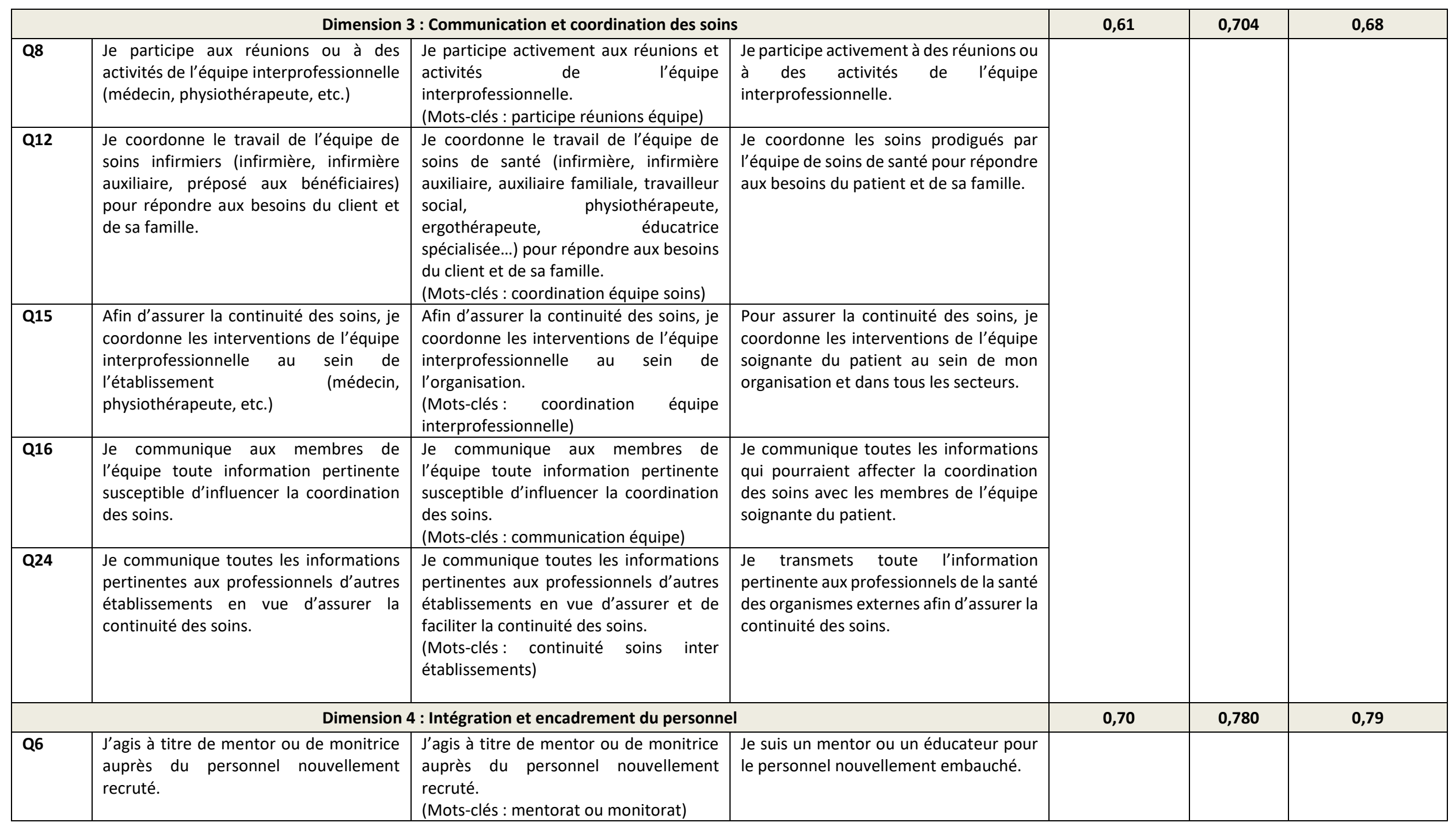




\begin{tabular}{|c|c|c|c|c|c|c|}
\hline Q9 & $\begin{array}{l}\text { Je participe à l'orientation et à } \\
\text { l'encadrement des stagiaires ou du } \\
\text { personnel nouvellement recruté. }\end{array}$ & $\begin{array}{l}\text { Je participe à l'orientation et à la } \\
\text { formation des étudiants en soins } \\
\text { infirmiers et/ou du personnel } \\
\text { nouvellement recruté. } \\
\text { (Mots-clés : orientation et formation de } \\
\text { la relève infirmière) }\end{array}$ & $\begin{array}{l}\text { Je suis impliqué dans l'orientation et la } \\
\text { formation des étudiants en soins } \\
\text { infirmiers et/ou du personnel } \\
\text { nouvellement embauché. }\end{array}$ & & & \\
\hline Q14 & $\begin{array}{l}\text { Je participe au développement ou à la } \\
\text { dispensation d'activités de formation à } \\
\text { l'équipe de soins, selon mes } \\
\text { compétences. }\end{array}$ & $\begin{array}{l}\text { Je participe à la conception, au } \\
\text { développement ou à la dispensation } \\
\text { d'activités de formation à l'équipe de } \\
\text { soins, et ce, selon mes compétences. } \\
\text { (Mots-clés : conception, développement } \\
\text { dispensation activités de formation) }\end{array}$ & $\begin{array}{l}\text { Je suis impliqué dans le développement } \\
\text { et la conduite d'activités de formation } \\
\text { pour l'équipe soignante, en fonction de } \\
\text { mes compétences. }\end{array}$ & & & \\
\hline Q20 & $\begin{array}{l}\text { Je participe à l'identification des besoins } \\
\text { de formation de mon unité. }\end{array}$ & $\begin{array}{l}\text { Je participe à l'identification des besoins } \\
\text { de formation de mon service. } \\
\text { (Mots-clés: identification besoin } \\
\text { formation) }\end{array}$ & $\begin{array}{l}\text { Je suis impliqué dans l'identification des } \\
\text { besoins de formation en cours d'emploi } \\
\text { pour mon organisation. }\end{array}$ & & & \\
\hline \multicolumn{4}{|c|}{ Dimension 5 : Optimisation de la qualité et de la sécurité des soins } & 0,66 & 0,762 & 0,75 \\
\hline Q3 & $\begin{array}{l}\text { Je participe au développement de la } \\
\text { pratique infirmière (ex. révision de } \\
\text { protocoles de soins, projets divers) }\end{array}$ & $\begin{array}{l}\text { Je participe au développement et } \\
\text { l'amélioration de la pratique infirmière } \\
\text { (ex. révision de protocoles de soins, } \\
\text { élaboration et révision d'ordonnance } \\
\text { collective, élaboration ou révision } \\
\text { d'outils cliniques...) } \\
\text { (Mots-clés: développement et } \\
\text { amélioration pratique infirmière) }\end{array}$ & $\begin{array}{l}\text { Je suis impliqué dans le développement } \\
\text { et l'amélioration de la pratique } \\
\text { infirmière pour refléter la pratique } \\
\text { fondée sur des données probantes. }\end{array}$ & & & \\
\hline Q7 & $\begin{array}{l}\text { Je signale les situations cliniques où je } \\
\text { perçois une lacune dans la qualité et la } \\
\text { sécurité des soins. }\end{array}$ & $\begin{array}{l}\text { Je signale les situations cliniques où je } \\
\text { perçois une lacune dans la qualité et la } \\
\text { sécurité des soins. } \\
\text { (Mots-clés: signalement des lacunes } \\
\text { cliniques) }\end{array}$ & $\begin{array}{l}\text { Je rapporte les situations cliniques dans } \\
\text { lesquelles je vois des déficiences dans la } \\
\text { qualité et la sécurité des soins. }\end{array}$ & & & \\
\hline Q19 & $\begin{array}{l}\text { Je propose des approches ou stratégies } \\
\text { pour améliorer la qualité et la sécurité } \\
\text { des soins lorsque j'identifie des lacunes. }\end{array}$ & $\begin{array}{l}\text { Je propose des approches ou des } \\
\text { stratégies afin d'améliorer la qualité et la } \\
\text { sécurité des soins lorsque j'identifie des } \\
\text { lacunes. } \\
\text { (Mots-clés: amélioration qualité et } \\
\text { sécurité soins) }\end{array}$ & $\begin{array}{l}\text { Lorsque j'ai identifié des possibilités } \\
\text { d'amélioration des pratiques de soins, je } \\
\text { recommande des approches ou des } \\
\text { stratégies pour améliorer la qualité et la } \\
\text { sécurité des soins dispensés au sein de } \\
\text { mon organisation. }\end{array}$ & & & \\
\hline
\end{tabular}




\begin{tabular}{|l|l|}
\hline Q23 & $\begin{array}{l}\text { Je participe à l'évaluation de la qualité et } \\
\text { de la sécurité des soins (utilisation } \\
\text { d'outils ou de systèmes d'évaluation de } \\
\text { la qualité des soins, questionnaire de } \\
\text { satisfaction, etc.). }\end{array}$ \\
\hline Q26 & $\begin{array}{l}\text { Je m'implique dans la mise à jour des } \\
\text { pratiques en vue d'améliorer la qualité et } \\
\text { la sécurité des soins. }\end{array}$ \\
\hline Q4 & $\begin{array}{l}\text { Je maintiens à jour mes connaissances } \\
\text { (ex. : lectures, activités scientifiques). }\end{array}$ \\
\hline Q13 & $\begin{array}{l}\text { Lors d'une modification de la pratique, je } \\
\text { partage avec l'équipe de soins infirmiers } \\
\text { les connaissances issues de la recherche. }\end{array}$ \\
\hline Q18 & $\begin{array}{l}\text { Je bonifie ma pratique en fonction des } \\
\text { nouvelles connaissances issues des } \\
\text { pratiques exemplaires et des projets de } \\
\text { recherche en sciences infirmières ou en } \\
\text { santé. }\end{array}$ \\
\hline Niveau de
\end{tabular}

Je participe à l'évaluation de la qualité et de la sécurité des soins (utilisation d'outils ou de systèmes d'évaluation de la qualité des soins, questionnaire de satisfaction, etc.)

(Mots-clés: évaluation qualité sécurité soins)

Je participe à la mise à jour des pratiques en vue d'améliorer la qualité et la sécurité des soins.

Mots-clés : mise à jour pratique)

Mise à jour et utilisation des connaissances

Je maintiens mes connaissances à jour

(ex. lectures, activités scientifiques, formation continue).

(Mots-clés : connaissances actualisées) Lors d'une modification de la pratique, je partage et fais la promotion des connaissances issues de la recherche avec l'équipe.

Mots-clés :

promotion

connaissances issues de la recherche)

Je bonifie ma pratique en fonction des nouvelles connaissances issues des pratiques exemplaires et de la recherche

en sciences infirmières ou en santé.

(Mots-clés : bonification de la pratique)

\begin{tabular}{|c|c|c|c|}
\hline $\begin{array}{l}\text { Je suis impliqué dans l'évaluation de la } \\
\text { qualité et de la sécurité des soins. }\end{array}$ & & & \\
\hline $\begin{array}{l}\text { Je mets à jour les pratiques cliniques } \\
\text { pour améliorer la qualité et la sécurité } \\
\text { des soins. }\end{array}$ & & & \\
\hline es & 0,70 & 0,617 & 0,66 \\
\hline Je garde mes connaissances à jour. & & & \\
\hline $\begin{array}{l}\text { Je partage avec l'équipe de soins les } \\
\text { connaissances issues de la recherche. }\end{array}$ & & & \\
\hline $\begin{array}{l}\text { J'améliore ma pratique en me basant sur } \\
\text { de nouvelles connaissances dérivées des } \\
\text { meilleures pratiques et de la recherche } \\
\text { en sciences infirmières ou en santé. }\end{array}$ & & & \\
\hline & & 0,823 & \\
\hline & & 0,769 & \\
\hline & & 0,807 & \\
\hline & 0,89 & 0,914 & 0,91 \\
\hline
\end{tabular}

Niveau de complexité 2 - Moyen (Q2, Q1, Q5, Q12, Q24, Q20, Q9, Q26, Q19, Q18)

Niveau de complexité 3 - Élevé (Q11, Q22, Q8, Q15, Q6, Q14, Q23, Q3, Q13)

Score global ÉEPI 
APPENDICE V

Certificat éthique 


\section{Santé \\ Québec \\ Comité central \\ recherche}

Montréal, le 2 mars 2018

Madame Lily Lessard

a/s de madame Mélanie Morin

Université du Québec à Rimouski-(Lévis)

Département des Sciences infirmières

1595, boulevard Alphonse-Desjardins

Lévis Québec G6V OA6

Objet: CCER 17-18 - 16 - Approbation finale.

L'étendue de la pratique des infirmières dans les services de proximité de la région

Madame Lessard,

Le Comité central d'éthique de la recherche du ministre de la Santé et des Services sociaux a évalué votre projet de recherche à sa réunion du $1^{\mathrm{er}}$ mars 2018 . Lors de cette réunion, les documents suivants ont été examinés

- Formulaire de demande d'évaluation d'un projet de recherche dûment complété signé et daté.

Protocole de recherche intitulé : L'étendue de la pratique des infirmières dans les services de proximité de la région de la et daté du 31 janvier 2018.

- Formulaire d'information et de consentement, daté 5 février 2018.

- Questionnaire en ligne via Google Form, non daté.

Document - Communication courriel aux gestionnaires pour l'annonce de l'étude, communication courriel pour recrutement, questions de recrutement préalables au questionnaire en ligne, Rappel par courriel, mot de remerciement par courriel, non daté.

- Curriculum vitæe de madame Lily Lessard.

- Curriculum vitæe de madame Mélanie Morin.

Suite à cette réunion, une approbation conditionnelle vous a été émise en date du $1^{\text {er }}$ mars 2018 . Vous nous avez soumis en date du $1^{\text {er }}$ mars 2018 , les documents suivants:

- Formulaire d'information et de consentement, daté $1^{\text {er }}$ mars 2018

- Document - Communication courriel aux gestionnaires pour l'annonce de l'étude, communication courriel pour recrutement, questions de recrutement préalables au questionnaire en ligne, Rappel par courriel, mot de remerciement par courriel, daté du $1^{\text {er }}$ mars 2018.

Vos réponses et les modifications apportées à votre projet de recherche ont fait l'objet d'une évaluation. Le tout ayant été jugé satisfaisant, j'ai le plaisir de vous informer que votre projet de recherche a été approuvé à l'unanimite par le Comité central d'éthique de la recherche du ministre de la Santé et des Services sociaux.

Les documents que le Comité central d'éthique de la recherche du ministre de la Santé et des Services sociaux a approuvés et que vous pouvez utiliser pour la réalisation de votre projet sont les suivants

- Protocole de recherche intitulé : L'étendue de la pratique des infirmières dans les services de proximité de la région de la et daté du 31 janvier 2018.

Formulaire d'information et de consentement, daté 2 mars 2018.

- Questionnaire en ligne via Google Form, date du 2 mars 2018.

- Document - Communication courriel aux gestionnaires pour l'annonce de l'étude, communication courriel pour recrutement, questions de recrutement préalables au questionnaire en ligne, Rappel par courriel, mot de

Comité central d'éthique de la recherche du ministre de la Santé et des Services sociaux

500 , rue Sherbrooke Ouest, bureau 800

Montréal, Québec, H3A 3C6

Télécopieur : (514) 873-8768

Courriel (Johane de Champlain) : jdechamplain@frq.gouv.qc.ca

Site du Comité : http://ethique. msss. gouv.qc. ca/lethique-de-la-recherche/comite-central.htm

Page 1 sur 
Approbation finale

remerciement par courriel, daté du 2 mars 2018

Cette approbation éthique est valide pour un an à compter du 2 mars 2018, date de l'approbation finale.

Deux mois avant la date d'échéance, vous devrez faire une demande de renouvellement auprès du Comité central d'éthique de la recherche du ministre de la Santé et des Services sociaux, en utilisant le document du Comité prévu à cet effet.

Dans le cadre du suivi continu, le Comité vous demande de vous conformer aux exigences suivantes en utilisant les formulaires du Comité prévus à cet effet :

1. De soumettre toute demande de modification au projet de recherche ou à tout document approuvé par le Comité pour la réalisation de votre projet.

2. De soumettre, dès que cela est portè à votre connaissance, tout nouveau renseignement ou toute modification à l'équilibre clinique susceptible d'affecter l'intégrité ou l'éthicité du projet de recherche, d'accroître les risques et les inconvénients pour les participants, de nuire au bon déroulement du projet ou d'avoir une incidence sur le désir d'un participant de continuer à participer au projet.

3. De soumettre, dès que cela est porté à votre connaissance et en lien avec la réalisation de ce projet, tout accident survenu dans votre site.

4. De soumettre, dès que cela est porté à votre connaissance, l'interruption prématurée du projet de recherche, qu'elle soit temporaire ou permanente.

5. De soumettre, dès que cela est porté à votre connaissance, tout problème constaté à la suite d'une activité de surveillance ou de vérification menée par un tiers et susceptible de remettre en question l'intégrité ou l'éthicité du projet de recherche

6. De soumettre, dès que cela est porté à votre connaissance, toute suspension ou annulation de l'approbation octroyée par un organisme de subvention ou de réglementation.

7. De soumettre, dès que cela est porté à votre connaissance, toute procédure en cours de traitement d'une plainte ou d'une allégation de manquement à l'intégrité ou à l'éthicité ainsi que des résultats de la procédure.

8. De soumettre, toute déviation au projet de recherche susceptible d'augmenter le niveau de risque ou susceptibles d'influer sur le bien-être du participant ou d'entacher le consentement du participant.

9. De soumettre une demande de renouvellement annuel de l'approbation du projet de recherche.

10. De soumettre le rapport de la fin du projet de recherche.

Vous pouvez obtenir les formulaires du Comité téléchargeables à partir du site web à l'adresse suivante http://ethique.msss.gouv.qc.ca/lethique-de-la-recherche/comite-central/documentation.html

Nous vous rappelons que la présente décision vaut pour une année et peut être suspendue ou révoquée en cas de non-respect de ces exigences.

De plus, nous vous rappelons que vous devez conserver pour une période d'au moins un an suivant la fin du projet, un répertoire distinct comprenant les noms, prénoms, coordonnées, date du début et de fin de la participation de chaque sujet de recherche.

Le Comité central d'éthique de la recherche du ministre de la Santé et des Services sociaux est institué par le ministre de la Santé et des Services sociaux aux fins de l'application de l'article 21 du Code civil du Québec et suit les règles émises par l'Énoncé de politique des trois conseils et les Bonnes pratiques cliniques de la $\mathrm{CIH}$.

Avec l'expression de nos sentiments les meilleurs.

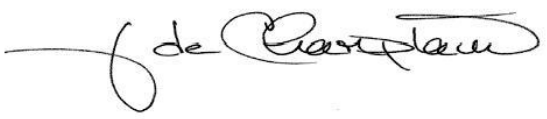

Johane de Champlain

Vice-présidente, Comité central d'éthique de la recherche

du ministre de la Santé et des Services sociaux

Jdec/

p. j.

Comité central d'éthique de la recherche du ministre de la Santé et des Services sociaux

500 , rue Sherbrooke Ouest, bureau 800

Montréal, Québec, H3A 3C6

eléphone : (514) $873-211$

Télécopieur : (514) 873-8768

Courriel (Johane de Champlain) : jdechamplain@frq.gouv.qc.ca

Site du Comité : http://ethique. misss, gouv.qc.ca/lethique-de-la-recherche/comite-central.htm

Page 2 sur 2 

APPENDICE VI

Autorisation du milieu de recherche 


\section{Québec}

Direction des services multidisciplinaires, qualité, évaluation, performance et éthique

\section{PAR COURRIEL}

Le 14 mars 2018

Mélanie Morin, infirmière clinicienne

Centre intégré de santé et de services sociaux

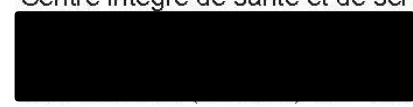

Objet : Autorisation de réaliser la recherche suivante : «Étude de l'étendue de la pratique infirmière dans les services de proximité de la région de la

Madame

Nous avons le plaisir d'autoriser la réalisation de la recherche identifiée en titre dans notre établissement.

Cette autorisation vous est accordée sur la foi des documents que vous avez déposés auprès de notre établissement, notamment la lettre du Comité central d'éthique de la recherche du ministère de la Santé et des Services sociaux, datée du $2^{\text {er }}$ mars 2018 qui établit que votre projet de recherche a fait l'objet d'un examen scientifique et d'un examen éthique dont le résultat est positif. Cette approbation éthique est valable jusqu'au 2 mars 2019. Si ce CER vous informe pendant le déroulement de cette recherche d'une décision négative portant sur l'acceptabilité éthique de cette recherche, vous devrez considérer que la présente autorisation de réaliser la recherche dans notre établissement est, de ce fait, révoquée à la date que porte l'avis du CER évaluateur.

Cette autorisation suppose également que vous respecterez les modalités énoncées ciaprès.

Notre établissement a reçu une copie de la version finale des documents se rapportant à la recherche, approuvée par le CER évaluateur.

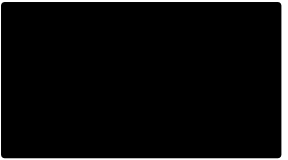


Cette autorisation de réaliser la recherche suppose également que vous vous engagez :

1) À vous conformer aux demandes du CER évaluateur, notamment pour le suivi éthique continu de la recherche;

2) À rendre compte au CER évaluateur et à la signataire de la présente autorisation du déroulement du projet, des actes de votre équipe de recherche, s'il en est une, ainsi que du respect des règles de l'éthique de la recherche;

3) À respecter les moyens relatifs au suivi continu qui ont été fixés par le CER évaluateur;

4) À conserver les dossiers de recherche pendant la période fixée par le CER évaluateur, après la fin du projet, afin de permettre leur éventuelle vérification;

5) À respecter les modalités arrêtées au regard du mécanisme d'identification des sujets de recherche dans notre établissement, à savoir :

- La tenue à jour et la conservation de la liste à jour des sujets de recherche recrutés dans notre établissement.

La présente autorisation peut être suspendue ou révoquée par notre établissement en cas de non-respect des conditions établies. Le CER évaluateur en sera alors informé.

Vous consentez également à ce que notre établissement communique aux autorités compétentes des renseignements personnels qui sont nominatifs au sens de la loi en présence d'un cas avéré de manquement à la conduite responsable en recherche de votre part lors de la réalisation de cette recherche.

En terminant, nous vous demandons de toujours mentionner dans votre correspondance au sujet de cette recherche, le numéro attribué au projet de recherche par le CER évaluateur.

Recevez, Monsieur, l'expression de nos salutations distinguées.

Le président-directeur-général,

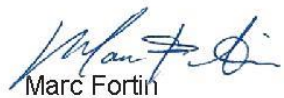



APPENDICE VII

Communication de recrutement 


\section{Communication courriel aux gestionnaires pour l'annonce de l'étude}

Bonjour,

Je m'adresse à vous afin de vous informer de la tenue d'un projet de recherche, lequel est réalisé sous la direction de Mme Lily Lessard (Ph. D.) professeure à I'Université du Québec à Rimouski (UQAR). Ce projet s'inscrit dans le cadre de ma formation à la maîtrise en sciences infirmières (option mémoire) à l'Université du Québec à Rimouski. Préoccupées par la réalité de la pratique actuelle des infirmières et infirmiers dans les services de proximité de la
nous souhaitons en évaluer l'étendue afin de dresser un portrait de la pratique infirmière dans les services
de proximité de notre région.

Les infirmières et les infirmières cliniciennes prodiguant des services à la clientèle dans les services de proximité du CISSS de la seront sollicitées afin de participer à l'étude. Par services de proximité, nous entendons les services infirmiers dispensés dans les groupes de médecine familiale et universitaire (GMF et GMF-U), les dispensaires et les centres locaux de services communautaires (CLSC) tels que les services de santé publique, périnatalité, soins de santé courants, équipe de suivi d'intensité variable en santé mentale dans la communauté et de soutien à domicile.

La participation à ce projet consiste à compléter un questionnaire en ligne d'une durée approximative de 20 minutes. Les participantes et participants à cette étude auront la chance de participer au tirage d'une carte cadeau d'une valeur de $100 \$$ chez Archambault.

La période allouée pour remplir le questionnaire débutera le (insérer la date) et prendra fin le (insérer la date 4 semaines plus tard). Vous pouvez communiquer avec moi par courriel si vous ou vos infirmières avez des questions en lien avec ce projet de recherche.

Je vous remercie d'avance pour votre précieuse collaboration,

Mélanie Morin, inf. B.Sc, Étudiante à la maîtrise en sciences infirmières Département des sciences infirmières, Université du Québec à Rimouski.

Boursière du MÉES et du RRISIQ

Melanie.Morin@uqar.ca

Document approuvé le 2 mars 2018 par le Comité central d'éthique de la recherche du ministre de la Santé et des services sociaux 


\section{Communication courriel pour recrutement}

Chères collègues infirmières et infirmiers,

Je m'adresse à vous dans le but de solliciter votre participation à mon projet de recherche, lequel est réalisé sous la direction de Mme Lily Lessard (Ph. D.) professeure à l'Université du Québec à Rimouski (UQAR). Cette étude s'inscrit dans le cadre de ma formation à la maîtrise en sciences infirmières (option mémoire) à l'Université du Québec à Rimouski.

Préoccupées par la réalité de la pratique actuelle des infirmières et infirmiers dans les services de proximité de la nous souhaitons en évaluer l'étendue afin de dresser un portrait de la pratique infirmière dans les services de proximité de notre région.

Les personnes recherchées pour participer à cette étude sont celles trav

les services de proximité du CISSS de la en tant qu'infirmières ou infirmier et prodiguant des services directs à la clientèle. Les services de proximité incluent les groupes de médecine familiale et universitaire (GMF et GMF-U), les dispensaires et les centres locaux de services communautaires (CLSC) tels que les services de santé publique, périnatalité, soins de santé courants, équipe de suivi d'intensité variable en santé mentale dans la communauté et de soutien à domicile.

Votre participation prendra environ 20 minutes et consiste essentiellement à remplir le questionnaire confidentiel en ligne dont le lien apparaît ci-dessous. Les participantes et les participants à cette étude auront la chance de participer au tirage d'une carte cadeau d'une valeur de $100 \$$ chez Archambault

Afin de préserver la confidentialité de vos réponses, aucune information nominative ne vous sera demandée dans le questionnaire. La date limite pour remplir le questionnaire est le (préciser la date)

Au besoin, vous pouvez me contacter par courriel si vous avez des questions en lien avec cette étude.

(Insérer le lien du questionnaire en ligne)

Je vous remercie d'avance pour votre participation,

Mélanie Morin, inf. B.SC., Étudiante à la maîtrise en sciences infirmières.

Département des sciences infirmières, Université du Québec à Rimouski.

Boursière du MÉES et du RRISIQ. 
Questions de recrutement préalables au questionnaire en ligne

Étude de l'étendue de la pratique infirmière dans les services de proximité de la région de la Côte-Nord Critères de sélection pour les répondants * Obligatoire

Détenez-vous un permis de pratique valide de l'OIIQ? * Marquez un seul ovale.

$\square$ Oui

$\square$ Non. Nous vous remercions de votre intérêt, mais vous ne répondez pas aux critères de sélection de cette étude. Merci de quitter le présent sondage.

Travaillez-vous au sein des services de proximité du CISSS de la côte-Nord soit les centres locaux de services communautaires(CLSC): services de périnatalité, santé publique, soutien à domicile, soins de santé courants et santé mentale, les dispensaires ou dans les groupes de médecine familiale (GMF, GMFU)? *

Marquez un seul ovale.

$\square$ Oui

$\square$ Non. Nous vous remercions de votre intérêt, mais vous ne répondez pas aux critères de sélection de cette étude. Merci de quitter le présent sondage.

Occupez-vous un poste ou un remplacement d'infirmière ou d'infirmière clinicienne qui offre des services directs à la clientèle?

Marquez un seul ovale.

$\square$ Oui

$\square$ Non. Nous vous remercions de votre intérêt, mais vous ne répondez pas aux critères de sélection de cette étude Merci de quitter le présent sondage. 


\section{Rappel par courriel}

Chères collègues infirmières et infirmiers,

Vous avez reçu dernièrement une invitation à participer à un questionnaire en ligne visant à évaluer l'étendue de la pratique au sein des services de proximité du CISSS de la

Cette étude s'inscrit dans le cadre de ma formation à la maîtrise en sciences infirmières à l'Universitè du Québec à Rimouski. À ce jour, nous avons reçu près de (insérer le nombre) questionnaires complétés, ce qui est très encourageant. Cependant, nous souhaitons obtenir un nombre supérieur de réponses afin d'assurer que les résultats de cette recherche soient représentatifs de la pratique des infirmières et infirmiers de la région de la _ En participant, vous contribuerez à une meilleure compréhension de la réalité votre pratique infirmière actuelle. Nous vous remercions à l'avance pour votre prècieuse collaboration et de prendre quelques minutes de votre temps pour compléter le sondage en ligne.

Le lien pour y accéder apparaît ci-dessous. Vous avez jusqu'au (insérer la date) pour compléter le questionnaire en ligne.

Si vous avez déjà complété et retourné le questionnaire en ligne, veuillez ignorer ce message. (Insérer le lien du questionnaire en ligne)

Merci d'avance pour votre temps,

Mélanie Morin, inf. B.Sc., Étudiante à la maîtrise en sciences infirmières. Département des sciences infirmières, Université du Québec à Rimouski. Boursière du MÉES et du RRISIQ.

Melanie.Morin@uqar.ca 
Mot de remerciement par courriel

Chères collègues infirmières et infirmiers,

Nous vous remercions d'avoir pris le temps de participer à l'étude visant à évaluer l'étendue de votre pratique au sein des services de proximité du CISSS de la Nous vous confirmons que le tirage de la carte cadeau a été réalisé et que le prix a été remis.

Encore une fois, merci de votre précieuse collaboration,

Mélanie Morin, inf. B.Sc., Étudiante à la maîtrise en sciences infirmières

Département des sciences infirmières, Université du Québec à Rimouski

Boursière du MÉES et du RRISIQ

Melanie.Morin@uqar.ca

Document approuvé le 2 mars 2018 par le Comité central d'éthique de la recherche du ministre de la Santé et des services sociaux 
APPENDICE VIII

Formulaire de consentement 


\section{FORMULAIRE D'INFORMATION ET DE CONSENTEMENT}

Titre du projet de recherche:

\section{Chercheur responsable du projet de recherche:}

Étudiante à la maîtrise :

Établissement participant :
Étude de l'étendue de la pratique infirmière dans les services de proximité de la région de la

Directrice de recherche : Lily Lessard, professeure à I'Université du Québec à Rimouski.

Mélanie Morin, infirmière clinicienne au et étudiante à la maîtrise en sciences infirmières à l'Université du Québec à Rimouski.

CISSS

\section{Introduction.}

Nous vous invitons à participer à un projet de recherche. Cependant, avant d'accepter de participer à ce projet de recherche, veuillez prendre le temps de lire, de comprendre et de considerer attentivement les renseignements qui suivent.

\section{Nature et objectifs du projet de recherche.}

Vous êtes invité à participer à ce projet de recherche parce que vous êtes une infirmière ou un infirmier travaillant au sein des services de proximité au CISSS de la définissent comme des services infirmiers dispensés dans les groupes de médecine familiale et universitaire, les cliniques médicales, les dispensaires et les centres locaux de services communautaires (CLSC) tels que les services de santé publique, périnatalité, soins de santé courants, équipe de suivi d'intensité variable en santé mentale dans la communauté et de soutien à domicile.

Ainsi, ce projet de recherche vise à mesurer l'étendue de la pratique des infirmières dans les services de proximité de la région de la et déterminer dans quelle mesure les caractéristiques individuelles et du travail sont susceptibles d'en influencer T'étendue.

Pour la réalisation de ce projet de recherche, nous comptons recruter de 40 à 133 infirmières et infirmiers, âgés de 18 à 65 .

3. Déroulement du projet de recherche.

Votre participation à ce projet de recherche consistera à répondre à un questionnaire en ligne d'une durée approximative de 20 minutes selon votre convenance et en fonction de vos disponibilités. Pour se faire, une version électronique préparée grâce à Google Form du Questionnaire de l'étendue de la pratique infirmière (D'Amours et al., 2012) sera utilisée.

4. Avantages associés au projet de recherche.

Vous ne retirerez aucun bénéfice personnel de votre participation à ce projet de recherche. Par ailleurs, les résultats obtenus contribueront à l'avancement des connaissances scientifiques dans ce domaine de recherche.

5. Inconvénients associés au projet de recherche.

Le principal inconvénient est relié au temps nécessaire pour compléter le questionnaire.

6. Participation volontaire et possibilité de retrait.

Votre participation à ce projet de recherche est volontaire. Vous êtes donc libre de refuser d'y participer. Vous pouvez également vous retirer de ce projet à n'importe quel moment, sans avoir à donner de raisons.

Le chercheur responsable de ce projet de recherche et le Comité central d'éthique de la recherche du ministre de la Santé et des Services sociaux peuvent mettre fin à ce projet de recherche, sans votre consentement. Cela peut se produire s'il existe des raisons administratives d'abandonner le projet. Si vous vous retirez du projet, l'information et le matériel déjà recueillis dans le cadre de ce projet seront néanmoins conservés, analysés ou utilisés pour assurer l'intégrité du projet.

8. Confidentialité.

Durant votre participation à ce projet de recherche, le chercheur responsable de ce projet ainsi que les membres de son personnel de recherche recueilleront, dans un dossier de recherche, les renseignements vous concernant et nécessaires pour répondre aux objectifs scientifiques de ce projet de recherche.

Ces renseignements comprendront des renseignements sociodémographiques, tels que votre âge, votre sexe, votre Formulaire d'information et de consentement approuvé le 2 mars 2018 par le Comité central d'éthique de la recherche du ministre de la Santé et des Services sociaux

CCER 17-18 - 16 - Participant majeur - Version du 2 mars 2018

Page 1 sur 2 
nombre d'années d'expérience en tant qu'infirmière, votre nombre d'années d'expérience au sein des services de proximité, votre niveau de formation, votre poste et votre statut d'emploi, votre milieu de pratique ainsi que vos réponses aux questions sur votre pratique infirmière.

Tous les renseignements recueillis demeureront confidentiels dans les limites prévues par la loi. De plus, votre employeur n'aura pas accès aux réponses de votre questionnaire. Afin de préserver votre identité et la confidentialité de ces renseignements, vous ne serez identifié que par un numéro de code. La clé du code reliant votre nom à votre dossier de recherche sera conservée par le chercheur responsable de ce projet de recherche.

Ces données de recherche seront conservées pendant au moins 5 ans par le chercheur responsable de ce projet de recherche. Les données de recherche pourront être publiées ou faire l'objet de discussions scientifiques, mais il ne sera pas possible de vous identifier.

À des fins de surveillance, de contrôle, de protection, de sécurité, votre dossier de recherche pourra être consulté par une personne mandatée par des organismes réglementaires ou par le Comité central d'éthique de la recherche du ministre de la Santé et des Services sociaux. Ces personnes et ces organismes adhèrent à une politique de confidentialité.

Vous avez le droit de consulter votre dossier de recherche pour verifier les renseignements recueillis et les faire rectifier au besoin.

\section{Compensation.}

Vous ne recevrez pas de compensation financière pour votre participation à ce projet de recherche, mais votre participation vous donne la chance de gagner une carte cadeau d'une valeur de $100 \$$ chez Archambault

\section{En cas de préjudice.}

En acceptant de participer à ce projet de recherche, vous ne renoncez à aucun de vos droits et vous ne libérez pas le chercheur responsable de ce projet de recherche, I'Université et l'établissement de leur responsabilité civile et professionnelle.

\section{Identification des personnes-ressources.}

Si vous avez des questions ou éprouvez des problèmes en lien avec le projet de recherche, ou si vous souhaitez vous en retirer, vous pouvez communiquer avec le chercheur responsable de ce projet de recherche ou avec une personne de l'équipe de recherche au numéro suivant, (418) 833-8800, poste 3350

Pour toute question concernant vos droits en tant que participant à ce projet de recherche ou si vous avez des plaintes ou des commentaires à formuler, vous pouvez communiquer avec vous pouvez communiquer avec le commissaire aux plaintes et à la qualité des services du Centre intégré de santé et de services sociaux

12. Surveillance des aspects éthiques du projet de recherche.

Le Comité central d'éthique de la recherche du ministre de la Santé et des Services sociaux a approuvé le projet de recherche et en assura le suivi. Pour toute information, vous pouvez communiquer avec madame Johane de Champlain, vice-présidente, au (514) 873.2114

\section{Consentement}

\section{Engagement du chercheur responsable de ce projet de recherche}

Je m'engage, avec l'équipe de recherche, à respecter ce qui a été convenu au formulaire d'information et de consentement.

\section{Consentement du participant.}

J'ai pris connaissance du formulaire d'information et de consentement. On m'a expliqué le projet de recherche et le présent formulaire d'information et de consentement. On a répondu à mes questions et on m'a laissé le temps voulu pour prendre une décision. Après réflexion, je consens à participer à ce projet de recherche aux conditions qui y sont énoncées et en soumettant ce questionnaire, je consens à participer à cette étude.

Formulaire d'information et de consentement approuvé le 2 mars 2018 par le Comité central d'éthique de la recherche du ministre de la Santé et des

CCER $17-18$ - 16 - Participant majeur - Version du 2 mars 2018. 

APPENDICE IX

Demande de modifications - Certificat éthique 
Santé

\section{Québec}

comité central

dethique de la

Montréal, le 24 avril 2018

Madame Lily Lessard

a/s de madame Mélanie Morin

Université du Québec à Rimouski-(Lévis)

Département des Sciences infirmières

1595, boulevard Alphonse-Desjardins

Lévis Québec G6V OA6

Objet: CCER 17-18 - 16 - Approbation de la demande de modification M1.

L'étendue de la pratique des infirmières dans les services de proximité de la région

Madame Lessard,

Vous avez soumis au Comité central d'éthique de la recherche du ministre de la Santé et des Services sociaux, par courriel, le 2 avril 2018, une demande de modification à votre projet de recherche cité en rubrique. À cet effet, vous avez soumis au Comité les documents suivants :

- Formulaire de demande d'approbation de modification dûment complété

- Protocole de recherche intitulé : L'étendue de la pratique des infirmières dans les services de proximité de la région de la $\quad$ et daté du 29 mars 2018, en mode révision.

Essentiellement, votre demande de modification vise à apporter des modifications à votre méthode de recrutement afin de vous permettre de rejoindre le plus grand nombre d'infirmières correspondant à votre population cible.

Votre demande de modification a fait l'objet d'une évaluation. Le tout étant jugé satisfaisant, j'ai le plaisir de vous informer que votre demande a été approuvée par le Comité. Ce faisant, le Comité vous autorise à utiliser, pour la réalisation de votre projet, le document suivant :

- Protocole de recherche intitulé : L'étendue de la pratique des infirmières dans les services de proximité de la région de la et daté du 29 mars 2018

Avec l'expression de nos sentiments les meilleurs.

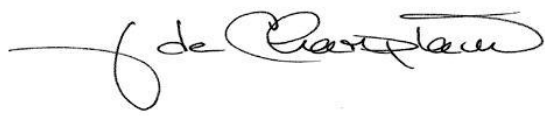

Johane de Champlain

Vice-présidente, Comité central d'éthique de la recherche

du ministre de la Santé et des Services sociaux

JdeC/

500 , rue Sherbrokique de la recherche

Montréal, Québec, H3A 3C6

Télécopieur : (514) $873-8768$

Courriel (Johane de Champlain) : jdechamplain@frq.gouv.qc.ca

Site du Comité : http://ethique, msss, gouv.qc.ca/lethique-de-la-recherche/comite-central.html

Page 1 sur 1 
APPENDICE $X$

Demande de renouvellement - Certificat éthique 


\section{Santé \\ Québec wit \\ comité central \\ recherch}

Montréal, le 19 février 2019

Madame Lily Lessard

a/s de madame Mélanie Morin

Université du Québec à Rimouski-(Lévis)

Département des Sciences infirmières

1595, boulevard Alphonse-Desjardins

Lévis Québec G6V 0A6

Objet: CCER 17-18 - 16 - Approbation de la demande de renouvellement R1

L'étendue de la pratique des infirmières dans les services de proximité de la région

Madame Lessard,

Vous avez soumis au Comité central d'éthique de la recherche du ministre de la Santé et des Services sociaux, par courriel, le 17 février 2019, une demande de renouvellement pour votre projet cité en rubrique.

Votre demande de renouvellement a fait l'objet d'une évaluation. Le tout étant jugé satisfaisant, j'ai le plaisir de vous informer que votre demande de renouvellement a été approuvée par le Comité central d'éthique de la recherche du ministre de la Santé et des Services sociaux.

Ainsi, vous pouvez poursuivre votre étude pour un an, et ce, à compter du 2 mars 2019 jusqu'au 2 mars 2020

Deux mois avant la date d'échéance vous devrez faire une demande de renouvellement auprès du Comité central d'éthique de la recherche du ministre de la Santé et des Services sociaux, en utilisant le document du Comité prévu à cet effet.

Nous vous rappelons que dans le cadre de son suivi continu, le Comité vous demande de vous conformer aux exigences suivantes en utilisant les formulaires du Comité prévus à cet effet :

1. De soumettre toute demande de modification au projet de recherche ou à tout document approuvé par le Comité pour la réalisation de votre projet.

2. De soumettre, dès que cela est porté à votre connaissance, tout nouveau renseignement ou toute modification à l'équilibre clinique susceptible d'affecter l'intégrité ou l'éthicité du projet de recherche, d'accroître les risques et les inconvénients pour les participants, de nuire au bon déroulement du projet ou d'avoir une incidence sur le désir d'un participant de continuer à participer au projet.

3. De soumettre, dès que cela est porté à votre connaissance et en lien avec la réalisation de ce projet, tout accident survenu dans votre site.

4. De soumettre, dès que cela est porté à votre connaissance, l'interruption prématurée du projet de recherche, qu'elle soit temporaire ou permanente.

5. De soumettre, dès que cela est portè à votre connaissance, tout problème constaté à la suite d'une activité de surveillance ou de vérification menée par un tiers et susceptible de remettre en question l'intégrité ou l'éthicité du projet de recherche

6. De soumettre, dès que cela est portè à votre connaissance, toute suspension ou annulation de l'approbation octroyée par un organisme de subvention ou de réglementation.

7. De soumettre, dès que cela est porté à votre connaissance, toute procédure en cours de traitement d'une plainte ou d'une allégation de manquement à l'intégrité ou à l'éthicité ainsi que des résultats de la procédure.

8. De soumettre, toute déviation au projet de recherche susceptible d'augmenter le niveau de risque ou susceptibles d'influer sur le bien-être du participant ou d'entacher le consentement du participant.

Comité central d'éthique de la recherche du ministre de la Santé et des Services sociaux

500 , rue Sherbrooke Ouest, bureau 800

Montréal, Québec, H3A 3C6

Téléphone : (514) 873-2114

Télécopieur : (514) 873-8768

Courriel (Johane de Champlain) : jdechamplain@frq.gouv.qc.ca

Site du Comité : http://ethique. msss. gouv. qc.ca/lethique-de-la-recherche/comite-central.html

Page 1 sur 2 
Renouvellement R1
CCER $17-18-16$

9. De soumettre une demande de renouvellement annuel de l'approbation du projet de recherche.

10. De soumettre le rapport de la fin du projet de recherche.

Vous pouvez obtenir les formulaires du Comité téléchargeables à partir du site web à l'adresse suivante : http://ethique.msss.gouv.qc.ca/lethique-de-la-recherche/comite-central/documentation.html

De plus, nous vous rappelons que vous devez conserver pour une période d'au moins un an suivant la fin du projet, un répertoire distinct comprenant les noms, prénoms, coordonnées, date du début et de fin de la participation de chaque sujet de recherche.

Finalement, je vous rappelle que la présente décision vaut pour une année et peut être suspendue ou révoquée en cas de non-respect de ces exigences.

Le Com ité central d'éthique de la recherche du ministre de la Santé et des Services sociaux est institué par le ministre de la Santé et des Services sociaux pour les fins de l'application de l'article 21 du Code civil du Québec et suit les règles émises par l'Énoncé de politique des trois conseils et les Bonnes pratiques cliniques de la $\mathrm{CIH}$

Avec l'expression de nos sentiments les meilleurs.

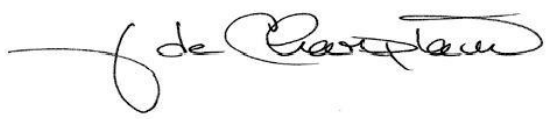

Johane de Champlain

Vice-présidente, Comité central d'éthique de la recherche

du ministre de la Santé et des Services sociaux

JdeC/ 


\section{RÉFÉRENCES}

Aggarwal et Hutchison. (2012). Toward a Primary Care Strategy for Canada. Canadian Foundation for Healthcare Improvement.

American nurses association. (2017). Scope of Practice. Repéré à https://www.nursingworld.org/practice-policy/scope-of-practice/

Aroke, E. N. (2014). Full Nursing Potential: A Concept Clarification. Nursing Forum, 49(4), 278-287. doi:10.1111/nuf.12096

Association des infirmières et infirmiers du Canada. (2009). Le leadership de la profession infirmière. Repéré à https://www.cna-aiic.ca/ /media/cna/pagecontent/pdf-fr/le-leadership-de-la-profession-infirmiere_enonce-deposition.pdf?la $=\mathrm{fr}$

Association des infirmières et infirmiers du Canada. (2014). Optimisation du rôle des infirmières et des infirmiers dans les soins primaires au Canada : Rapport final.

Baranek, P. M. (2005). A review of scopes of practice of health professions in Canada: A balancing act. Toronto: Health Council of Canada.

Bauer L. etBodenheimer T. (2017). Expanded roles of registered nurses in primary care delivery of the future. Nursing Outlook, 65 (5), pp. 624-632.

Besner, J., Doran, D., McGillis Hall, L., Giovannetti, P., Girard, F., Hill, W., \& Morrison, J. (2005). A systematic approach to maximizing nursing scopes of practice. Ottawa: Canadian Institutes of Health Research.

Birks, M., Smithson, J., Lindsay, D., \& Davis, J. (2018). How registered nurses determine their scope of practice: a cross-sectional study. Contemporary Nurse: A Journal for the Australian Nursing Profession, 54(6), 578-591. doi:101 080/10376178.2018.1520605

Borgès Da Silva, R., Brault, I., Pineault, R., Chouinard, M.-C., Prud'homme, A., \& D'Amour, D. (2018). Nursing Practice in Primary Care and Patients' Experience of Care. Journal of Primary Care \& Community Health, 9, N.PAGN.PAG. doi:101 177/2 150131917747186 
Braithwaite, S. E. L. (2016). Measuring scope of practice enactment among primary care registered nurses in Ontario. (Mémoire de maîtrise inédit). Queen's University, Kingston, Ontario. Repéré à https://qspace.library.queensu.ca/jspui/bitstream/handle/1974/14998/Braithwa ite_Suzanne_EL_September2016_MNSc.pdf? sequence=1\&isAllowed=y

Büscher, A., Sivertsen, B., \& White, J. (2009). Nurses and midwives: a force for health: World Health Organization (WHO). Repéré à http://www.euro.who.int/_data/ assets/pdf_file/0019/114157/E93980.pdf

Careau E, Brière N, Houle N, Dumont S, Maziades J, Paré L, et al. (2014). Continuum de pratiques de collaboration interprofessionnelle en santé et services sociaux. Guide explicatif. Repéré à http://www.rcpi.ulaval.ca/fileadmin/media/Coffre_a_outils/CONTINUUM_v 15_web_1_.pdf

Chabal, S. (2014). Les principaux biais à connaître en matière de recueil d'information. Les fiches En lignes de la lettre du CEDIP, fiche 62.

Chávez EC, Yoder LH. (2015). Staff nurse clinical leadership: a concept analysis. Nursing Forum,50(2), 90-100.

College of Registered Nurses of British Columbia. (2014). Scope of practice for registered nurses: standards, limits, conditions. (Publication No.433). Vancouver, BC: Auteur. à https://www.crnbc.ca/Standards/Lists/StandardResources/433ScopeforRegiste redNurses.pf

College of Registered Nurses of Manitoba. (2010). Understanding Scope of Practice. Winnipeg, Manitoba : Auteur. Repéré à https://www.crnm.mb.ca

College of Registered Nurses of Nova Scotia. (2009). A discussion paper on scope of nursing practice for registered nurses in Nova Scotia. Halifax. NouvelleÉcosse. Récupéré http://citeseerx.ist.psu.edu/viewdoc/download?doi=10.1.1.610.7643\&rep=rep $1 \&$ type $=$ pdf

Comité de la formation des infirmières. (2014). Avis sur la formation infirmière initiale et sécurité des soins. Tiré de : https://www.oiiq.org/documents/20147/1306159/avis-cfi-securite-soins2014.pdf 
Comité de la formation des infirmières. (2016). Avis sur les compétences en sécurité des patients dans la formation infirmière. Repéré à https://www.oiiq.org/documents/20147/1306159/avis-competences-securitepatients-ca20161006-07.pdf

Commissaire à la santé et au bien-être [CSBE]. (2014). Perceptions et expériences de soins de la population: Le Québec comparé. Résultats de l'enquête internationale sur les politiques de santé du Commonwealth Fund de 2013. Québec, QC. Gouvernement du Québec. Repéré à http://www.csbe.gouv.qc.ca/fileadmin/www/2013/CWF/CSBE_Rapport_Com monwealth_Fund_2013.pdf?goback=.gde_3944916_member_5831413891673 923584\#!

Commissaire à la santé et au bien-être. (2017a). La performance du système de santé et de services sociaux Québécois 2016. Québec, QC. Gouvernement du Québec. Repéré à https://www.csbe.gouv.qc.ca/fileadmin/www/2017/PerformanceGlobale/CSB E_RapportGlobal_2016_ACCESS.pdf

Commissaire à la santé et au bien-être. (2017 b). Utilisation des urgences en santé mentale et en santé physique au Québec. Québec, QC. Gouvernement du Québec. Repéré https://www.csbe.gouv.qc.ca/fileadmin/www/2017/Urgences/CSBE_Rapport_ Urgences_2017.pdf

Conseil international des infirmières. (2013). Position statement: Scope of nursing practice. ICN, Geneva.

D’Amour, D., Dubois, C., Dery, J., Clarke, S., Tchouaket, E., Blais, R., \& Rivard, M., (2012). Measuring Actual Scope of Nursing Practice A New Tool for Nurse Leaders. The Journal of Nursing Administration. 42(5), 248-255. doi:10.1097/NNA.0b013e31824337f4

Déry, J., (2013). L'étendue effective de la pratique d'infirmières en pédiatrie : ses déterminants et son influence sur la satisfaction professionnelle. (Thèse de doctorat inédite). Université de Montréal, Montréal, QC. Repéré à http://hdl.handle.net/1866/10843

Fares, S., Clinton, M., \& Younan, L. (2018). The first Arabic version of the Actual Scope of Nursing Practice Scale: Psychometric evaluation. Journal of Nursing Management (John Wiley \& Sons, Inc.), 26(8), 1059-1065. doi:10.1111/jonm.12635 
Fealy, G. M., Rohde, D., Casey, M., Brady, A. M., Hegarty, J., Kennedy, C., McNamara, M., O'Reilly, P., \& Prizeman, G. (2015). Facilitators and barriers in expanding scope of practice: findings from a national survey of Irish nurses and midwives. Journal of Clinical Nursing (John Wiley \& Sons, Inc.), 24(2324), 3615-3626. doi:10.1111/jocn.12980

Gauthier, J., Haggerty, J., Lamarche, P., Lévesque, J.-F., Morin, D., Pineault, R. \& Sylvain, H. (2009). Entre adaptabilité et fragilité : les conditions d'accès aux services de santé des communautés rurales et éloignées. Repéré à https://www.inspq.qc.ca/pdf/publications/1014_ConditionsAccesServSanteCo mmunRurales.pdf

Gouvernement du Canada. (2018). Le système des soins de santé du Canada. Repéré à https://www.canada.ca/fr/sante-canada/services/systeme-soins-sante/rapportspublications/regime-soins-sante/canada.html

Gouvernement du Québec. (2016). Comité patronal de négociation du secteur de la santé et des services sociaux. Conventions collectives nationales 2016-2020. Nomenclature en vigueur. Repéré à http://wpp01.msss.gouv.qc.ca/app1/N02/

Gouvernement du Québec. (2017). Plan stratégique du ministère de la Santé et des Services sociaux du Québec 2015-2020. Repéré à https://cdncontenu.quebec.ca/cdn-contenu/adm/min/sante-services-sociaux/publicationsadm/plan-strategique/PL_17-717-01W_MSSS.pdf

Groves, S. K., Burns, N. \& Gray, J., R. (2013). The practice of nursing research : appraisal, synthesis, and generation of evidence. St. Louis, Miss. : Elsevier/Saunders. 7th ed.

Halcomb, E. J., Salamonson, Y., Davidson, P. M., Kaur, R., \& Young, S. A. M. (2014). The evolution of nursing in Australian general practice: a comparative analysis of workforce surveys ten years on. BMC Family Practice, 15, 1-10. doi:10.1186/1471-2296-15-52

Halcomb, E., \& Ashley, C. (2016). Australian primary health care nurses most and least satisfying aspects of work. Journal of Clinical Nursing (John Wiley \& Sons, Inc.), 26(3-4), 535-545. doi:10.1111/jocn.13479

Halcomb, E. J., Peters, K., \& Davies, D. (2013). A qualitative evaluation of New Zealand consumers perceptions of general practice nurses. BMC Family Practice, 14(1), 26-32. doi:101 186/1471-2296-14-26 
Heppell-Cayouette, M., Maranda, J. (2016). Intégration de la relève infirmière dans les milieux cliniques. Communication du Comité Jeunesse au congrès de 1'OIIQ 2016. https://www.oiiq.org/sites/default/files/uploads/evenements/congres2016/pdf/i ntegration-releve-congres-2016-a11.pdf

Holt, J., Zabler, B., \& Baisch, M. J. (2014). Evidence-based characteristics of nursemanaged health centers for quality and outcomes. Nursing Outlook, 62(6), 428439. doi:10.1016/j.outlook.2014.06.005

Houweling, S. T., Kleefstra, N., Van Hateren, K. J. J., Groenier, K. H., Jong, B. M., \& Bilo, H. J. G. (2011). Can diabetes management be safely transferred to practice nurses in a primary care setting? A randomised controlled trial. Journal of Clinical Nursing (John Wiley \& Sons, Inc.), 20(9-10), 1264-1272. doi:10.1111/j.1365-2702.2010.03562.x

Institut de la statistique du Québec. (2015). Effectif du réseau de la santé et des services sociaux selon certaines catégories du personnel infirmier, régions sociosanitaires et ensemble du Québec, 2009-2010 à 2013-2014. Repéré le 2016-02-29, http://www.stat.gouv.qc.ca/statistiques/profils/comp_interreg/tableaux/infirmi ers.htm

Institut de la statistique du Québec. (2019). Profils statistiques par région et MRC géographiques. Repéré à http://www.stat.gouv.qc.ca/statistiques/profils/region_00/region_00.htm

Institute of Medicine. (2010). The future of nursing: Leading change, advancing health. Washington, DC: The National Academies Press.

Jacobson, P. M. (2012). Evidence Synthesis for the effectiveness of interprofessional teams in primary care. Canadian Nurses Association.

Kunic, R. J., \& Jackson, D. (2013). Transforming nursing practice: barriers and solutions. AORN Journal, 98(3), 235-248. doi:10.1016/j.aorn.2013.07.003

Lafleur, C., (2016). L'étendue effective de pratique des infirmières œuvrant en santé mentale et le niveau de formation. (Mémoire de maîtrise inédit). Université de Montréal. Montréal, QC. Repéré à https://papyrus.bib.umontreal.ca/xmlui/bitstream/handle/1866/16292/Lafleur_ Martine_2016_memoire.pdf?sequence=4\&isAllowed=y 
Lampron, K. (2010). L'étendue de la pratique chez les infirmières cliniciennes et les infirmières. (Mémoire de maîtrise inédit). Université de Montréal, QC. Repéré à https://papyrus.bib.umontreal.ca/xmlui/handle/1866/4493

Lessard L, Morin D, Sylvain H. (2008). Comprendre les équipes et le travail en équipe. L'infirmière Canadienne, 9(3), 10-11.

Lévesque, J.-F., Pineault, R., Provost, S., Tousignant, P., Couture, A., Da Silva, R. B., \& Breton, M. (2010). Assessing the evolution of primary healthcare organizations and their performance (2005-2010) in two regions of Québec province: Montréal and Montérégie. BMC Family Practice, 11, 95-107. doi:10.1186/1471-2296-11-95

Loi sur les infirmières et infirmiers du Québec (chapitre I-8).

Maranda J. et Lessard L., 2017. Prendre sa place : Réflexion sur le leadership clinique des infirmières cliniciennes au Québec. L'infirmière clinicienne, vol. 14(1), 4552. Repéré à http://revueinfirmiereclinicienne.uqar.ca/Parutions/documents/MarandaetLessard_2017_I nfirimiereClinicienne_Vol14no1_V2pp45-52_000.pdf

Mathieu, L. (2019). La pleine occupation du champ d'exercices : un leitmotiv en 2019. Repéré à https://www.oiiq.org/la-pleine-occupation-du-champ-d-exercice-unleitmotiv-en-2019?inheritRedirect $=$ true $\&$ redirect $=\% 2 \mathrm{~F}$

MSSS. (2008). Programme national de soutien clinique : Volet préceptorat. Cadre de référence. Repéré https://publications.msss.gouv.qc.ca/msss/fichiers/2008/08-526-01.pdf

MSSS. (2013). Rapport du président du Groupe de travail sur la formation de la relève infirmière. Repéré http://publications.msss.gouv.qc.ca/msss/fichiers/2013/13-945-01W.pdf

MSSS. (2018). O-7.2 - Loi modifiant l'organisation et la gouvernance du réseau de la santé et des services sociaux notamment par l'abolition des agences régionales Repéré à http://legisquebec.gouv.qc.ca/fr/ShowDoc/cs/O-7.2

MSSS. (2019). Plan stratégique 2019-2023 Repéré à https://cdncontenu.quebec.ca/cdn-contenu/adm/min/sante-services-sociaux/publicationsadm/plan-strategique/PL_19-717-02W_MSSS.pdf 
Ministère des Affaires municipales et de l'habitation. (2018). Cartothèque : Region09. Gouvernement du Québec. Repéré https://www.mamh.gouv.qc.ca/fileadmin/cartes/region/09.pdf

Morin, M. et L. Lessard. (2019). L'étendue effective de la pratique des infirmières dans les services de proximité en région éloignée. Recherche en soins infirmiers, Septembre (138), 75-93.

Nicol-Clavet, N. (2017). La pratique infirmière en groupe de médecine de famille dans le cadre du Plan Alzheimer Québec. (Mémoire de maîtrise inédit). Université de Sherbrooke, Sherbrooke, QC. Repéré à https://savoirs.usherbrooke.ca/bitstream/handle/11143/11567/Nicol_Clavet_N oemie_MA_2017.pdf?sequence=3\&isAllowed=y

Normandin, P.-A. (2016). Difficile accès aux soins de santé au Québec. La Presse. Repéré à http://www.lapresse.ca/actualites/sante/201602/10/01-4949100difficile-acces-aux-soins-de-sante-au-quebec.php

Oelke, N. D., White, D., Besner, J., Doran, D., Hall, L. M., \& Giovannetti, P. (2008). Nursing workforce utilization: an examination of facilitators and barriers on scope of practice. Nursing Leadership (1910-622X), 21(1), 58-71.

Oelke, N. D., Besner, J., \& Carter, R. (2014). The evolving role of nurses in primary care medical settings. International Journal of Nursing Practice (John Wiley \& Sons, Inc.), 20(6), 629-635. doi:10.1111/ijn.12219

Ordre des infirmières et infirmiers du Québec. (2004). Une situation inacceptable qui perdure depuis trop longtemps. Repéré à http://collections.banq.qc.ca/ark:/52327/bs1973323

Ordre des infirmières et infirmiers du Québec. (2006). Le plan thérapeutique infirmier : La trace des décisions cliniques de l'infirmière. Repéré à https://www.oiiq.org/documents/20147/237836/222_doc.pdf

Ordre des infirmières et infirmiers du Québec. (2011). La formation continue pour la profession infirmière au Québec Repéré à https://www.oiiq.org/documents/20147/1306047/270NS-Normeprofessionnelle-WEB.pdf 
Ordre des infirmières et infirmier du Québec. (2014a). Mémoire : Optimiser la contribution des infirmières et infirmiers pour améliorer l'accès aux soins, assurer la qualité et la sécurité des soins et contrôler les coûts. Repéré à https://www.oiiq.org/documents/20147/237836/3417-memoire-commissionrevision-programmes.pdf

Ordre des infirmières et infirmier du Québec. (2014 b). Champ d'exercice et activités réservées de l'infirmière Exemples en soins de santé de proximité (Soins dans la communauté). Repéré à https://www.oiiq.org/documents/20147/237836/schema-7-soins-santeproximite.pdf

Ordre des infirmières et infirmier du Québec. (2016a). Le champ d'exercices et les activités réservées des infirmières et infirmiers. 3e édition. Repéré à https://www.oiiq.org/documents/20147/1306047/1466-exercice-infirmieractivites-reservees-web+\%282\%29.pdf/84aaaa05-af1d-680a-9be129 fcde $8075 \mathrm{e} 3$

Ordre des infirmières et infirmier du Québec. (2016 b). Définition du PTI. Repéré à https://www.oiiq.org/pratique-professionnelle/encadrement-de-lapratique/outils-cliniques/plan-therapeutique-infirmier/definition-du-pti

Ordre des infirmières et infirmier du Québec. (2018). L'OIIQ s'inquiète de la sécurité des soins infirmiers. Repéré à https://www.oiiq.org/l-oiiq-s-inquiete-de-lasecurite-des-soins-infirmiers 1 ?inheritRedirect=true

Ordre des infirmières et infirmier du Québec. (2019a). Ordonnances et protocoles nationaux. Repéré à https://www.oiiq.org/pratiqueprofessionnelle/encadrement-de-la-pratique/outils-cliniques/ordonnancescollectives/ordonnances-et-protocoles-nationaux?inheritRedirect=true

Ordre des infirmières et infirmiers du Québec. (2019 b). Le Nord, une pratique qui donne des ailes. Repéré à https://www.oiiq.org/veronique-bonin-le-nord-unepratique-qui-donne-des-ailes?inheritRedirect=true).

Ordre des infirmières et des infirmiers du Québec et Collège des médecins du Québec. (2015). Guide explicatif conjoint : Prescription infirmière. Repéré à https://www.oiiq.org/documents/20147/237836/1451_doc.pdf 
Paquet, C. (2018). Chute des demandes d'admission en soins infirmiers - « Triste et désolant » selon le syndicat des infirmières. Journal Le Manic. Repéré à https://www.lemanic.ca/2018/03/21/chute-des-demandes-dadmission-ensoins-infirmiers-triste-et-desolant-selon-le-syndicat-des-infirmieres/

Pepin, J., Larue, C., Allard, É. \& Ha, L. (2015). La discipline infirmière : Une contribution décisive aux enjeux de santé. Secrétariat international des infirmières et infirmiers de l'espace francophone.

Parkinson, A. M., \& Parker, R. (2013). Addressing chronic and complex conditions: what evidence is there regarding the role primary healthcare nurses can play? Australian Health Review, 37(5), 588-593. doi:10.1071/AH12019

Poghosyan, L., Boyd, D. R., \& Clarke, S. P. (2016). Optimizing full scope of practice for nurse practitioners in primary care: A proposed conceptual model. Nursing Outlook, 64(2), 146-155. doi:10.1016/j.outlook.2015.11.015

Radwin LE, Castonguay D, Keenan CB, Hermann C. (2016). An Expanded Theoretical Framework of Care Coordination Across Transitions in Care Settings. Journal of Nursing Care Quality, 31(3), 269-74.

Randall, S., Crawford, T., Currie, J., River, J., \& Betihavas, V. (2017). Impact of community based nurse-led clinics on patient outcomes, patient satisfaction, patient access and cost effectiveness: A systematic review. International Journal of Nursing Studies, 73, 24-33. doi:10.1016/j.ijnurstu.2017.05.008

Roy, C. (2015). Stratégies à privilégier pour permettre aux infirmières et infirmiers de déployer pleinement leur étendue de pratique. (Mémoire de maîtrise inédit). Université de Montréal. Montréal, QC. Repéré à https://papyrus.bib.umontreal.ca/xmlui/bitstream/handle/1866/13141/Roy_Car oline_2015_travail_dirige.pdf?sequence=2\&isAllowed $=\mathrm{y}$

Savard, I. et J. Rodrigue. (2001). Trop ou trop peu d'omnipraticiens au Québec ? Le médecin $d u \quad$ Québec, 36(10), 107-114. Repéré à https://lemedecinduquebec.org/Media/70598/107114SavardRodrigue1001.pdf

Secrétariat international des infirmières et infirmiers de l'espace francophone. (2015). Mémoire: La qualité des soins et la sécurité des patients: Une priorité mondiale. Repéré à https://www.sidiief.org/wp-content/uploads/SIDIIEF-Mmoire-Qualit-des-soins.pdf 
Smolowitz, J., Speakman, E., Wojnar, D., Whelan, E.-M., Ulrich, S., Hayes, C., \& Wood, L. (2015). Role of the registered nurse in primary health care: Meeting health care needs in the 21st century. Nursing Outlook, 63(2), 130-136. doi:10.1016/j.outlook.2014.08.004

Tremblay, L. (2018). La pleine occupation de notre champ d'exercices : un enjeu profondément actuel qui est l'affaire de tous. Perspective infirmière, 15(3), p.7. Repéré à https://www.oiiq.org/la-pleine-occupation-de-notre-champ-dexercice-un-enjeu-profondement-actuel-qui-est-l-affaire-de-tous

Vancol-Fabre, F. (2015). L'étendue optimale de la pratique de l'infirmière et l'infirmière clinicienne en première ligne. (Mémoire de maîtrise inédit). Université de Montréal. Montréal, QC. Repéré à https://papyrus.bib.umontreal.ca/xmlui/bitstream/handle/1866/13140/Vancol_ Fable_Fabiola_2015_Travail_Dirige.pdf? sequence $=2 \&$ isAllowed $=y$

Villegas, W.J. \& Allen, P.E. (2012). Barriers to advanced practice registered nurse scope of practice: issue analysis. Journal of Continuing Education in Nursing, 43 (9), 403-409. doi.org/10.3928/00220124-20120716-30

White, D., Oelke, N. D., Besner, J., Doran, D., Hall, L. M., \& Giovannetti, P. (2008). Nursing scope of practice: descriptions and challenges. Nursing Leadership (1910-622X), 21(1), 44-57.

Younan, L., Clinton, M., Fares, S., \& Samaha, H. (2019). The translation and cultural adaptation validity of the Actual Scope of Practice Questionnaire. Eastern Mediterranean Health Journal = La Revue De Sante De La Mediterranee Orientale = Al-Majallah Al-Sihhiyah Li-Sharq Al-Mutawassit, 25(3), 181-188. doi:10.26719/emhj.18.02 